\title{
Cis-regulatory similarities in the zebrafish and human pancreas uncover potential disease-related enhancers
}

R. Bordeira-Carriço ${ }^{1,2, \#}$, J. Teixeira ${ }^{1,2,3, \#}$, M. Duque ${ }^{1,2,3, \#}$, M. Galhardo ${ }^{1,2,4}$, D. Ribeiro $^{1,2}$, R. Dominguez-Acemel ${ }^{5}$, P. N. Firbas ${ }^{5}$, J. J. Tena ${ }^{5}$, A. Eufrasio, ${ }^{1,2}$, J. Marques $^{1,2}$, F. Ferreira ${ }^{1,2}$, T. Freitas ${ }^{1,2}$, F. Carneiro ${ }^{6,7}$, J. L. Goméz-Skarmeta ${ }^{5}$ and J. Bessa $^{1,2^{*}}$

*Corresponding author

jose.bessa@ibmc.up.pt

\# Equal contribution

1- Instituto de Biologia Molecular e Celular (IBMC), Universidade do Porto, Porto, Portugal.

2- Instituto de Investigação e Inovação em Saúde (i3S), Universidade do Porto, Porto, Portugal.

3- Doctoral program in Molecular and Cell Biology (MCbiology), Instituto de Ciências Biomédicas Abel Salazar (ICBAS), Universidade do Porto, Porto, Portugal.

4- Instituto de Ciências, Tecnologias e Agroambiente - CIBIO, Universidade do Porto, Portugal.

5-Centro Andaluz de Biología del Desarrollo (CABD), Universidad Pablo de Olavide, Spain.

6- Departamento de Patologia, Faculdade de Medicina, Universidade do Porto, Porto, Portugal.

7- Institute of Molecular Pathology and Immunology, IPATIMUP, University of Porto, Porto, Portugal. 


\section{Introductory paragraph}

The pancreas is a central organ for human diseases that have a dramatic societal burden, such as pancreatic cancer and diabetes ${ }^{1,2}$. Non-coding cis-regulatory elements (CREs) of DNA control gene $\operatorname{expression}^{3,4}$, being required for proper pancreas function. Most disease-associated alleles ${ }^{5,6}$ are noncoding, often overlapping with $\mathrm{CREs}^{5}$, suggesting that alterations in these regulatory sequences contribute to human pancreatic diseases by impairing gene expression. However, functional testing of CREs in vivo is not fully explored. Here we analysed histone modifications, transcription, chromatin accessibility and interactions, to identify zebrafish pancreas CREs and their human functional equivalents, uncovering disease-associated sequences across species. We found a human pancreatic enhancer whose deletion impairs the tumour suppressor gene ARIDIA expression, conferring a potential tumour suppressor role to this non-coding sequence. Additionally, we identified a zebrafish ptfla distal enhancer which deletion generates pancreatic agenesis, demonstrating the causality of this condition in humans ${ }^{7}$ and the interspecies functional equivalency of enhancers.

\section{Results}

The pancreas of zebrafish, a vertebrate model suitable for genetic manipulation ${ }^{8}$, shares many similarities with the human pancreas, including transcription factors (TFs) operating in similar genetic networks of pancreas development and function ${ }^{9,10}$. We observed that these similarities are extensible to organ structure (Fig.1a) and cellular composition (SupplementaryFig.1), suggesting that shared genetic networks might operate through equivalent sets of CREs in both species.

To identify CREs active in the zebrafish adult pancreas, we performed ChIP-seq for $\mathrm{H} 3 \mathrm{~K} 27 \mathrm{ac}^{11}$, a key histone modification associated to active enhancers and ATAC-seq ${ }^{12}$, to identify regions of open chromatin. We have also performed $\mathrm{HiChIP}^{13}$ against $\mathrm{H} 3 \mathrm{~K} 4 \mathrm{me} 3^{14}$ to determine active promoters interacting with the uncovered enhancers (Fig.1b). We found 14753 putative active enhancers, mostly in intergenic regions (57.8\%), and 23298 putative active promoters corresponding to 9848 genes (Fig.1c; SupplementaryTable1-3). To identify a subset of pancreas enhancers with higher tissue-specificity, we asked which of them are inactive in whole embryos (dome to 48hpf), finding that $7115(48.2 \%)$ are active only in the differentiated adult pancreas (PsE; Fig.1d; SupplementaryTable4) while the remaining 7638 (51.8\%) are also active during embryonic development (DevE). Interestingly, DevE presented 4 clusters (C1-4) with different activity dynamics during development (Fig.1d; SupplementaryFig.2; SupplementaryTable5).

Pancreatic enhancers should activate the expression of genes in the pancreas. To test this, we identified the nearest genes to each putative pancreas enhancer ${ }^{15}$, observing that genes nearby PsE 
are enriched for exocrine pancreas expression $(\mathrm{p}<4.27 \mathrm{e}-9$; $\quad$ SupplementaryFig.3a; SupplementaryTable6-7), detected by in situ hybridization. These results contrast with DevE, further suggesting a higher tissue-specificity of PsE. To improve the enhancer to gene association, we used $\mathrm{H} 3 \mathrm{~K} 4 \mathrm{me} 3 \mathrm{HiChIP}$ to detect interactions between active promoters and putative enhancers in the zebrafish adult pancreas (Fig.1b; SupplementaryTable8), and used RNA-seq to evaluate transcription. We found that PsE-associated genes have a higher average expression in a variety of pancreatic cell types when comparing to all transcribed genes, contrasting with transcription in the muscle (Fig.2a, SupplementaryTable9). Similar results were obtained when analysing genes associated to the remaining clusters of pancreatic enhancers (PsEs+DevE, DevE and C1-4; SupplementaryFig.3). Performing a similar assay using the transcriptome of whole zebrafish embryos from 18 developmental stages ${ }^{16}$, genes associated to DevE have shown an increased average expression comparing to all transcribed genes, with a similar dynamic to the enhancer activation during development (Fig.2b; SupplementaryFig.4). These results suggest that DevE enhancers control gene expression in the adult differentiated pancreas and during development.

To determine if the identified regulatory sequences are active pancreatic enhancers, we have performed in vivo reporter assays for 10 regions with strong H3K27ac signal and 7 with low levels of this mark (Fig.2c-d; Supplementary Fig.5). From the first set, we have found that 5 out of 10 tested sequences (H3K27ac: $-\log _{10}(p$-value $\left.) \geq 35\right)$ are pancreatic enhancers $(50 \%$; Fig.2c-d and SupplementaryTable10). In contrast, from the regions with low H3K27ac signal, only 1 out of 7 tested (14\%, Supplementary Fig.5) showed strong and reproducible pancreatic enhancer activity $\left(-\log _{10}(p-\right.$ value)<35). These results validate the robustness of the enhancers prediction based on chromatin state.

We observed that out of 14753 putative zebrafish pancreas enhancers, only $12.49 \%(n=1842)$ could be directly aligned to the human genome ${ }^{17}$ (Fig.3a; SupplementaryTable11), a similar proportion found in developmental enhancers (11.36\%; 7326 out of 64498), with both groups sharing similar PhastCons conservation scores (Fig.3b; SupplementaryFig.6a; SupplementaryTable11). Next, we wanted to discern if the zebrafish putative pancreas enhancers that align to the human genome also overlap with H3K27ac signal from human pancreas. Although only a minority of interspecies aligned sequences shared H3K27ac signal (Total pancreas data set: 229 out of 1842; PsE: 116 out of 1052; DevE: 113 out of 790), there is a clear enrichment comparing to random sequences (Fig.3c), although not showing a higher average sequence conservation score (Fig.3b). These results suggest that pancreatic enhancer function is not a strong constraint for sequence conservation.

Then, we wanted to assess whether functionally equivalent pancreatic CREs might exist between human and zebrafish, despite an overall lack of sequence conservation. To explore this possibility, we analysed if the human ortholog genes coupled to each cluster of zebrafish enhancers 
were enriched for human pancreatic diseases. Such enrichment was observed for the clusters of late development and adult (PsE, C3 and C4; Fig.3d; SupplementaryTable12). Subsequently, we searched for super enhancers active in the pancreas of both species, finding 275 in zebrafish and 875 in humans (SupplementaryTable13). Gene ontology for putative target genes showed a similar enrichment for transcriptional regulation in both species and several of these genes corresponded to the same orthologs (32 out of 271-zebrafish, SupplementaryFig.6b-c), some having important pancreatic functions, such as INSR and GATA6 (Fig.3e; SupplementaryFig.6d). We further asked if human and zebrafish enhancers might operate similarly, using equivalent TFs. To test this, we performed motif discovery for TF binding sites (TFBS) in regions of open chromatin identified by ATAC-seq ${ }^{12}$, within the 14753 pancreas enhancers, finding several TFBS for known pancreatic TFs (ZP; Fig.3f; SupplementaryTable14). We have performed a similar analysis using available human whole pancreas datasets (HP) and, to establish comparisons, for zebrafish embryos (D80, dome and 80\%epiboly; 24HPF, $24 \mathrm{hpf}$ ) and human heart ventricle (V). Selecting the top 140 enriched motifs from each dataset, we observed that the majority of the common motifs were found in zebrafish (ZP) and human (HP) pancreas datasets (ZP,HP:98; ZP,D80:63; HP,D80:61), while comparisons with the human ventricle (V) showed that ZP,HP was the second largest group (Fig.3g; SupplementaryFig.7a-b). Additionally, selecting 25 motifs from TFs known to be required for pancreas function or development, we found that the majority of those were within the ZP,HP overlapping datasets, regardless of the compared groups (SupplementaryFig.7c-e). These results suggest that the same set of TFs might operate in zebrafish and human pancreas enhancers. Overall, these results argue in favour of interspecies functional equivalency of enhancers. To better address this hypothesis, we focused on the regulatory landscape of aridlab, the orthologue of human ARIDIA, a tumoursuppressor gene known to be associated with cancer in several organs, including pancreas ${ }^{18,19}$. We identified one robust zebrafish pancreatic enhancer (zArid1abE3, Fig.4a), validated in vivo (Fig.4b; SupplementaryFig.8), that interacts with the promoter of aridlab (Fig.4a; SupplementaryFig.9). Additionally, we detected a human/zebrafish syntenic block containing the zebrafish zArid1abE3 enhancer and a human pancreatic CRE (hArid1abE3). Enhancer assays for hArid1abE3 demonstrated its ability to drive expression in the pancreas, suggesting a functional equivalency to the zebrafish zArid1abE3 enhancer (Fig.4b; SupplementaryFig.8). To study the impact of this human enhancer in ARID1A expression, we deleted hArid1abE3 enhancer in hTERT-HPNE cell line, a human pancreatic duct cell line, through CRISPR-cas9 system (SupplementaryFig.10), using as a control a deletion in an unrelated genomic region ${ }^{20}$. We observed lower ARID1A expression upon deletion of hAridE3 than in the control (Fig4c-e and SupplementaryFig10), suggesting that the loss of this enhancer might interfere with the DNA-damage response, with possible implications in the increased risk for pancreatic cancer ${ }^{21,22}$. 
To further evaluate the interspecies functional equivalency of enhancers, we have focused in the human locus of PTF1A, known to be controlled by a distal downstream enhancer whose deletion was associated to pancreatic agenesis ${ }^{7}$ (Fig.5a; hPtflaE3). Concomitantly, we detected a novel putative zebrafish enhancer located distally downstream of ptfla (zPtflaE3), apart from two previously identified proximal enhancers (zPtflaE1 and zPtflaE2) ${ }^{23}$. zPtflaE3 interacts with the promoter of ptfla, observed by Hi-ChIP and 4C-seq (Fig.5a; SupplementaryFig.9), and could correspond to the functional equivalent enhancer associated with pancreatic agenesis in humans (hPtflaE3). In vivo enhancer assays for zPtflaE3 and hPtflaE3 have shown that both are pancreatic enhancers able to drive expression in the same cell types (acinar, duct and pancreas progenitor cells), with a more robust expression in progenitor cells (Fig.5b; SupplementaryFig.11-12), suggesting that these two enhancers share some regulatory information. This is further supported by binding sites for FOXA2 and PDX1 in the human hPtflaE3, also predicted to bind to the zebrafish zptflaE3 (SupplementaryFig.13). To further validate these results, we have generated genomic deletions in the zPtflaE3 sequence, observing a reduction in the pancreatic progenitor domain, resulting in pancreatic hypoplasia (Fig.5d; SupplementaryFig.14-16), compatible with loss-of-function of ptfla in zebrafish $^{23}$ and the proposed loss of hPtflaE3 function in humans ${ }^{7}$. These results demonstrate that zebrafish and humans share a functional equivalent distal enhancer of $P T F 1 A$, clarifying the causality of pancreatic agenesis upon enhancer disruption in humans.

Cis-regulatory mutations and variations are associated with pancreatic cancer and diabetes 5,24-27, however the in vivo implications of these genetic changes are yet unknown. Here we explore the chromatin state of the zebrafish pancreas, to uncover pancreatic enhancers and establish comparisons with humans, to predict and model human pancreas disease-associated enhancers. We found that, although most of the zebrafish pancreas enhancers show no sequence conservation with human pancreas enhancers, they share many TFBS and their target genes are enriched for human pancreas diseases. These results suggest the existence of functionally equivalent enhancers in zebrafish and humans, as proposed for other tissues and species ${ }^{28}$. We illustrate this finding for the regulatory landscape of $A R I D 1 A$, a tumour-suppressor gene active in the pancreas ${ }^{21,29}$ and other tissues $^{18}$, finding a human pancreatic enhancer, whose deletion impairs ARID1A expression, defining a locus for non-coding mutations that might increase the risk for pancreatic cancer. We further validated these findings for one enhancer of $P T F 1 A^{30}$, where both zebrafish and human enhancers share regulatory information and biological requirement, showing a decrease in the pancreatic progenitor domain in its absence and, as a consequence, developing pancreatic agenesis. In summary, we show that transcriptional cis-regulation of the human and zebrafish adult pancreas have a high similarity, allowing the functional exploration of cis-regulatory sequences in zebrafish, with the potential of translation to human pancreatic diseases. 


\section{Acknowledgements:}

This study was supported by the European Research Council (ERC) under the European Union's Horizon 2020 research and innovation programme (grant agreement no. ERC-2015-StG-680156ZPR and ERC-2016-AdG-740041-EvoLand to JLG-S). JB acknowledges Fundação para a Ciência e a Tecnologia (FCT), for a FCT Investigator position (Grant IF/00654/2013). JLG-S also acknowledges the Spanish Ministerio de Economía y Competitividad (Grant BFU2016-74961-P), the Marató TV3 Fundacion (Grant 201611) and the institutional grant Unidad de Excelencia María de Maeztu (MDM-2016-0687 to the Department of Gene regulation and morphogenesis of Centro Andaluz de Biología del Desarrollo. RBC was funded by FCT (ON2201403-CTO-BPD), IBMC (BIM/04293-UID991520-BPD) and EMBO (Short-Term Fellowship). JTx (SFRH/BD/126467/2016), MD (SFRH/BD/135957/2018), AE (SFRH/BD/147762/2019), and FF (PD/BD/105745/2014) are PhD fellows from FCT. MG was supported by the EnvMetaGen project via the European Union's Horizon 2020 research and innovation programme (grant agreement 668981). Authors acknowledge the support of i3S Scientific Platform Advanced Light Microscopy, member of the national infrastructure PPBI-Portuguese Platform of BioImaging (supported by POCI01-0145-FEDER-022122). Authors acknowledge Carla Oliveira (Microenvironments for New Therapies) for statistical support, Catarina Meireles and Emilia Cardoso from the Translational Cytometry (TraCy) Scientific Platform, Mafalda Sousa for the automated cell analysis, Paula Magalhães and Tânia Meireles from Cell Culture and Genotyping Scientific Platform, Guilherme Cardoso from the Histology Service from Ipatimup Diagnostics, the I3s hpc facility used for data processing, in particular to Andre Torres, and finally Isabel Guedes for support in the maintenance of zebrafish lines. We also acknowledge, from CABD, the support from Elisa de la Calle-Mustienes and Sandra Jimenez Gancedo on ChiP-seq, Ana Fernandez-Minñán on 4C-seq and Ensieh Farahani on ATAC-seq protocols.

\section{Author Contributions}

JB designed the study with input of RBC and JLGS. JB coordinated the project. JB and JLGS supervised the work. All authors contributed for the development and discussion of the work. RBC obtained biological material and generated next-generation sequencing data from zebrafish pancreas. JT, MG, RDA, PNF and JJT performed computational analyses and data interpretation. RBC, MD, AE and DR performed enhancer-assays in zebrafish, from plasmid generation to transgenesis, and Crispr-Cas9 in zebrafish. MD, DR and AE performed immunohistochemistry, microscopy acquisition and analysis. JTx performed transfection, Crispr-Cas9 and image acquisition in human cell lines with support of FF. FC contributed with histology of human pancreas. TF and JM contributed for plasmid and zebrafish lines generation. JB wrote the manuscript with input from all authors. 


\section{Material and Methods \\ I) Experimental procedures}

\section{Zebrafish stocks, husbandry, breeding and embryo culture}

Adult zebrafish $\mathrm{AB} / \mathrm{TU}$ WT strains, transgenic and mutant lines were maintained at $26-28^{\circ} \mathrm{C}$ under a $10 \mathrm{~h}$ dark/14h light cycle in a recirculating housing system according to standard protocols ${ }^{31}$. Embryos were grown at $28^{\circ} \mathrm{C}$ in $\mathrm{E} 3$ medium [ $5 \mathrm{mM} \mathrm{NaCl}, 0.17 \mathrm{mM} \mathrm{KCl}, 0.33 \mathrm{mM} \mathrm{CaCl}_{2} \cdot 2 \mathrm{H}_{2} \mathrm{O}, 0.33 \mathrm{mM} \mathrm{MgSO}_{4} \cdot 7 \mathrm{H}_{2} \mathrm{O}$ and $0.01 \%$ methylene blue (Sigma-Aldrich), pH 7.2] or E3 supplemented with 0.01\% PTU (1-phenyl-2-thiourea) ${ }^{32}$. For the in vivo enhancer assays, embryos were anesthetized by adding tricaine (MS222; ethyl-3-aminobenzoate methanesulfonate, \#E10521-10G, Sigma-Aldrich) to the medium and selected by the internal positive control of transgenesis. For the establishment of transgenic and mutant zebrafish lines, embryos were microinjected, selected, bleached and grown until adulthood. Adult F0s were outcrossed with WT adults and the offspring screened for the internal control of transgenesis and the pattern of expression of the regulatory element, or for the respective mutations, by genotyping. In vivo reporter lines, $\operatorname{Tg}$ (ela:mCherry) and $\operatorname{Tg}($ sst:mCherry), were used to label the exocrine and endocrine domain, respectively. The i3S animal facility and this project were licensed by Direcção Geral de Alimentação e Veterinária $(D G A V)$ and all the protocols used for the experiments were approved by the i3S Animal Welfare and Ethics Review Body.

\section{Cell culture}

hTERT-HPNE (intermediary cells formed during acinar-to-ductal metaplasia; ATCC CRL-4023) cells were cultured in a $5 \% \mathrm{CO}_{2}$-humidified chamber at $37^{\circ} \mathrm{C}$ in DMEM (1x, $4.5 \mathrm{~g} / \mathrm{L}$ D-glucose with pyruvate; \#D6429, Gibco, ThermoFisher Scientific), supplemented with 10\% fetal bovine serum (\#BCS0615, biotecnomica), 10ng/mL human recombinant EGF (\#11343406, Immunotools) and 750ng/mL puromycin (\#P8833-25MG, Sigma-Aldrich) in TC Dish 100 (SARSTEDT). When cells reached to 90\% of confluence, they were split using TrypLE Express (\#12604-021, Gibco, ThermoFisher Scientific; approximately $0.5 \mathrm{~mL}$ per $10 \mathrm{~cm} 2$ ).

\section{ChIP-seq}

Whole pancreas was dissected from 25 adult zebrafish ( $\sim 50 \times 10^{6}$ cells), kept on ice in PBS with 1x Complete Proteinase Inhibitor (\#11697498001, Roche), fixed in 2\% formaldehyde (\#F1635-500ML, Sigma-Aldrich) for $10 \mathrm{~min}$, and stored at $-80^{\circ} \mathrm{C}$. ChIP was performed as previously described for zebrafish embryos ${ }^{33}$ with minor alterations. Cell lysis was performed on ice, using a $15 \mathrm{~mL}$ Tenbroeck Homogenizer, in cell lysis buffer [10mM Tris-HCl pH7.5, 10mM NaCl, 0.5\% NP-40, 1x Complete Proteinase Inhibitor (\#11697498001, Roche)] for 15 min. Nuclei were washed and re-suspended in nuclei lysis buffer $(50 \mathrm{mM}$ Tris-HCl pH7.5, 10mM EDTA, $1 \%$ SDS, 1x Complete Proteinase Inhibitor (\#11697498001, Roche)). Chromatin was sheared using a BioruptorPlus (Diagenode) device with the following cycling conditions: 10 min high-30 sec on, $30 \mathrm{sec}$ off; $15 \mathrm{~min}$ on ice; $10 \mathrm{~min}$ high-30 sec on, $30 \mathrm{sec}$ off. The sonicated chromatin had a size in the range of 100-500 
bp and was incubated overnight at $4^{\circ} \mathrm{C}$ with the anti-H3K27ac antibody (\#ab4729, Abcam). Samples were incubated for $1 \mathrm{~h}$ at $4^{\circ} \mathrm{C}$ with Dynabeads Protein $\mathrm{G}$ for Immunoprecipitation (\#10003D, Invitrogen, ThermoFisher Scientific). Final DNA was purified with MinElute (\#28004, Qiagen) and sequenced on Illumina HiSeq 2000 platform.

\section{ATAC-seq}

ATAC-seq was performed as previously described ${ }^{34}$, with minor changes. Whole pancreas was dissected from 2-3 adult zebrafish. Following cell lysis, 50000-10000 nuclei were submitted to tagmentation with Nextera DNA Library Preparation Kit (\#FC-121-1030, Illumina). ATAC-seq libraries were amplified KAPA HiFi HotStart PCR Kit (Roche) with the primers Ad1, Ad2.2 and Ad2. ${ }^{12}$, and further purified with PCR Cleanup Kit (\#28104, Qiagen).

\section{C-seq}

4C-seq was performed as previously described ${ }^{34}$, with minor alterations. Whole pancreas was dissected from

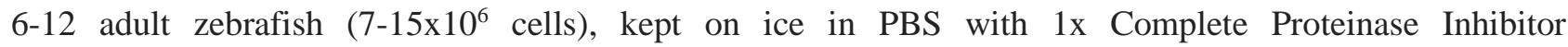
(\#11697498001, Roche), fixed in 2\% formaldehyde (\#F1635-500ML, Sigma-Aldrich) for 10 min, and stored at $-80^{\circ} \mathrm{C}$. Cell lysis was performed on ice, with a $15 \mathrm{~mL}$ Tenbroeck Homogenizer, not exceeding $10 \mathrm{~min}$. Ligation was performed with 60U T4 DNA Ligase (\#EL0012, ThermoFisher Scientific). The restriction enzymes used were DpnII (\#R0543M, NEB) and Csp6I (\#ER0211, ThermoFisher Scientific) for the first and second cuts, respectively. Chromatin was purified by Amicon Ultra-15 Centrifugal Filter Device (Milipore). 4C libraries were prepared for Illumina sequencing by the Expand Long Template Polymerase (Roche) with primers targeting the TSSs of each gene and including Illumina adapters (SupplementaryTable16). Final PCR products were purified with the High Pure PCR Product Purification Kit (Roche) and AMPure XP PCR purification kit (Agencourt AMPure XP).

\section{HiChIP-seq}

HiChIP-seq was performed as previously described ${ }^{13}$, with minor alterations. Whole pancreas was dissected, fixed in 1\% formaldehyde (\#F1635-500ML, Sigma-Aldrich) and cells lysed as described for 4C-seq. Immediately after lysis, samples were washed with HiChIP Wash Buffer (Tris-HCl pH 8 50mM, NaCl $50 \mathrm{mM}$, EDTA $1 \mathrm{mM}$ ). Chromatin was sonicated using the BioruptorPlus (Diagenode) with the following cycling conditions: $10 \mathrm{~min}$ high-30 sec on, $30 \mathrm{sec}$ off; $15 \mathrm{~min}$ on ice, to obtain a size in the range of 100-500 bp. Samples were incubated with anti-H3K4me3 antibody (\#AB8580, Abcam) and Dynabeads Protein G for Immunoprecipitation (\#10003D, Invitrogen, ThermoFisher Scientific) and purified with DNA Clean and Concentrator columns (Zymo Research). Up to $150 \mathrm{ng}$ of the DNA was then biotinylated with Streptavidin C1 beads (ThermoFisher Scientific). Tagmentation was performed using Nextera DNA Library Preparation Kit (\#FC-121-1030, Illumina). Libraries were amplified using NEBNext® High-Fidelity 2X PCR Master Mix 
(\#M0541S, NEB) with primers Ad1, Ad2.23 and Ad2.24 ${ }^{12}$. The final product was purified with DNA Clean and Concentrator kit (Zymo Research).

\section{Generation of plasmids for enhancer assays}

Putative enhancer sequences were selected based on the overlap between H3K27Ac ChIP-seq and ATAC-seq signal in non-coding regions within the landscape of each pancreas-relevant gene. Sequences were PCR amplified from zebrafish genomic DNA using the primers in SupplementaryTable16 (Sigma-Aldrich), with the proof-reading iMax ${ }^{\mathrm{TM}}$ II DNA polymerase (INtRON Biotechnology) following the manufacturer's instructions for a standard $20 \mu \mathrm{l}$ PCR reaction. PCR products were visualized by electrophoresis on an $1 \%$ agarose gel, the bands excised, purified with NZYGelpure kit (NZYTech) and cloned into the entry vector pCR®8/GW/TOPO (\#250020 Invitrogen, ThermoFisher Scientific) according with manufacturer's instructions. The vectors were then recombined into the destination vectors $\mathrm{Z}_{4} 8^{35}$, for transient enhancer assays, and $\mathrm{ZED}^{36,37}$, for stable transgenic lines, using Gateway® LR Clonase ${ }^{\circledR}$ II Enzyme mix (Invitrogen, ThermoFisher Scientific), following manufacturer's instructions.

Standard chemical transformation was performed with MultiShotTM FlexPLate Mach1TM T1R (Invitrogen, ThermoFisher Scientific), grown O.N. at $37^{\circ} \mathrm{C}$. Vector selection was performed with $100 \mu \mathrm{g} / \mathrm{ml}$ Spectinomycin (Sigma-Aldrich) in the growth medium for the $\mathrm{pCR} \otimes 8 / \mathrm{GW} / \mathrm{TOPO}$ vectors, or $100 \mu \mathrm{g} / \mathrm{ml}$ Ampicillin (Normon) for the Z48 and ZED vectors. Plasmids were extracted with NZYMiniprep kit (NZYTech) and confirmed by Sanger sequencing, using M13 forward and M13 reverse primers for pCR®8/GW/TOPO vector, and GW reverse primer for Z48 and ZED vector. Final plasmids were purified with phenol/chloroform and concentration was determined by NanoDrop 1000 Spectrophotometer (ThermoFisher Scientific).

\section{In vitro mRNA synthesis, Microinjection and Transgenesis}

Z48 and ZED zebrafish lines were generated through TOL2-mediated transgenesis ${ }^{38}$. TOL2 cDNA was transcribed by Sp6 RNA polymerase (ThermoFisher Scientific) after Tol2-pCS2FA vector linearization with NotI restriction enzyme (Anza, Invitrogen, ThermoFisher Scientific). TOL2 mRNA was purified as previously described ${ }^{36}$. One-cell stage embryos were injected with $1 \mathrm{~nL}$ solution containing $25 \mathrm{ng} / \mu \mathrm{L}$ of transposase mRNA, $25 \mathrm{ng} / \mu \mathrm{L}$ of phenol/chloroform purified plasmid (Z48 or ZED), and $0.05 \%$ phenol red.

\section{Cas9 target design, sgRNA synthesis and mutant generation}

Small guide RNAS (sgRNAs) targeting regions flanking zPtf1aE3 were designed using the Crisprscan software $^{39}$ (SupplementaryTable16). Oligonucleotides $(1,5 \mu \mathrm{L}$ at $100 \mu \mathrm{M}$ each, from Sigma-Aldrich) were annealed in vitro by incubation at $95^{\circ} \mathrm{C}$ for $5 \mathrm{~min}$ in $2 \mathrm{x}$ Annealling Buffer (10mM Tris, pH7.5-8.0, 50mM NaCL, 1mM EDTA) followed by slow cooling at RT, and inserted into 100ng of pDR274 vector (\#42250, Addgene) previously cut with BsaI (Anza, Invitrogen, ThermoFisher Scientific; 1:10). The pDR274 vectors carrying sgRNA sequences were linearized with HindIII, purified with phenol/chloroform and transcribed with 
T7 RNA polymerase (ThermoFisher Scientific). Final sgRNAs were purified as described previously ${ }^{36}$. One cell-stage zebrafish embryos were co-injected with two sgRNAs (40 ng/ $\mu \mathrm{l}$ each) and Cas 9 protein $(300 \mathrm{ng} / \mu \mathrm{l})$. Zebrafish mutant lines for zPtf1aE3 deletion were generated using the combinations sgRNA1+sgRNA2 and sgRNA3+sgRNA2 (SupplementaryTable16). Enhancer deletions in zebrafish were detected with PCR using HOT FIREPol DNA Polymerase (Solis BioDyne) with the flanking primers used to amplify the enhancers (SupplementaryTable16). PCR products were visualized by electrophoresis in $2 \%$ agarose gel and confirmed by Sanger sequencing. The mutations were further verified in the F1 mutants by sequencing.

\section{Crispr-Cas9 in human cell lines}

Four single-guide sequences named sg1, sg2, sg3, sg4, targeting hArid1abE3 enhancer were designed (SupplementaryTable16). sg1 and sg3 were designed upstream of the enhancer, while sg2 and sg4 were designed downstream of the enhancer. Two complementary oligonucleotides containing the single-guide sequences and BbsI ligation adapters were synthesized by Sigma. Two single-guide sequences for deletion of non-active region (based on $\mathrm{H} 3 \mathrm{~K} 27 \mathrm{ac}$ ), named ng1 and ng2, were used as negative control of the experiment ${ }^{20}$. Oligonucleotides were annealed in T4 Ligation Buffer (ThermoFisher Scientific). sgRNA was cloned into the BbsI-linearized pSpCas9-T2A-GFP (\#48138, Addgene) (sg1, sg3, ng1) and pU6-(BbsI)_CBh-Cas9-T2AmCherry (\#64324, Addgene) (sg2, sg4, ng2) vectors using T4 Ligase (ThermoFisher Scientific). The plasmid DNA was produced with Plasmid Midi Kit (Qiagen).

hTERT-HPNE cells were seeded in 6-well plates $\left(1.1 \times 10^{5}\right.$ cells/well, at early passage number) and transfected ( 70-90\% of confluency) using combinations: $n g 1+\mathrm{ng} 2 ; \mathrm{sg} 1+\mathrm{sg} 2 ; \mathrm{sg} 3+\mathrm{sg} 4$. The transfection $(1.5 \mu \mathrm{g}$ of each sgRNA plasmid) was performed using lipofectamine 3000 (ThermoFisher Scientific), according to the manufacture instructions. Then, we changed to fresh culture medium after $24 \mathrm{~h}$. Three independent replicates of the transfection were performed. After $48 \mathrm{~h}$ of recovery, the cells were used in sub-sequent experiments.

\section{Nucleic acid extraction from zebrafish and human cell lines}

Genomic DNA was extracted from whole zebrafish embryos at $24 \mathrm{hpf}$, after removal of chorion, with the standard phenol/-chloroform DNA extraction, and used as template for PCR amplification in order to genotype the tested conditions (SupplementaryTable16). The DNA samples were resuspended in $20 \mu 1$ of TE buffer with RNase [10mM Tris, pH 8.0; 1mM EDTA pH 8.0 and $100 \mu \mathrm{g} / \mathrm{ml}$ RNAse (Sigma-Aldrich)], incubated for 1 hour at $37^{\circ} \mathrm{C}$, and stored at $-20^{\circ} \mathrm{C}$.

Genomic DNA from hTERT-HPNE cells was extracted 48h after transfection and used as template for PCR amplification in order to genotype the tested conditions (SupplementaryTable16).

RNA was extracted from zebrafish embryos with 500 $\mu 1$ TRIzol (Invitrogen, ThermoScientific), following the manufacturer's instructions. Samples were incubated $30 \mathrm{~min}$ at $37^{\circ} \mathrm{C}$ with $1 \mu 1$ DNAse I (ThermoScientific), $1 \mu \mathrm{l} 10 \mathrm{x}$ reaction buffer and $0.5 \mu \mathrm{NZY}$ Ribonuclease Inhibitor $(40 \mathrm{U} / \mu \mathrm{l})$ at $0.05 \mu \mathrm{l} / \mu \mathrm{l}$ final concentration. After 
adding $1 \mu \mathrm{l}$ EDTA $50 \mathrm{mM}$ per $1 \mu \mathrm{g}$ of estimated RNA, final volume was completed to $60 \mu \mathrm{l}$ with $\mathrm{H} 2 \mathrm{O}$, phenolchloroform standard purification was performed and the RNA stored at $-80^{\circ} \mathrm{C}$.

\section{Immunohistochemistry in zebrafish embryos and human cell lines}

Zebrafish embryos/larvae were euthanized by prolonged immersion in 200-300 mg/L tricaine (MS222; ethyl3-aminobenzoate methanesulfonate, \#E10521-10G, Sigma-Aldrich). Whenever necessary the chorion was removed, and the zebrafish were fixed in formaldehyde 4\% (\#F1635-500ML, Sigma-Aldrich) for 1h at RT (812dpf larvae) or O.N. at $4^{\circ} \mathrm{C}$ (48hpf embryos). Permeabilization was carried out by incubation with $1 \%$ Triton $\mathrm{X}-100$ in PBS for $1 \mathrm{~h}$ at RT, followed by blocking with $5 \%$ bovine serum albumin (BSA) in $0.1 \%$ Triton X100 for $1 \mathrm{~h}$ at RT. Zebrafish were incubated with the primary antibody diluted in blocking solution at $4^{\circ} \mathrm{C}$ O.N., and then incubated with the secondary antibody plus DAPI (1:1000, Invitrogen, ThermoFisher Scientific) diluted in blocking solution for 4 hours at RT. After each antibody incubation, embryos were washed 6 times in PBS-T (0.5 \% Triton X-100 in PBS-1x) 5 minutes at RT. Embryos were stored in 50\% Glycerol/PBS at $4^{\circ} \mathrm{C}$ before microscopy slides preparation in mounting medium (50\% Glycerol/PBS). Images were acquired with confocal microscope Leica-SP5II (Leica Microsystems, Germany) and processed by ImageJ software. Primary antibodies: rabbit anti-Amylase (1:50, \#A8273-1VL, Sigma-Aldrich), mouse anti-Alcam (1:50, \#ZN-8, DSHB) and mouse anti-Nkx6.1 (1:50, \#F55A10, DSHB). Secondary antibodies: goat anti-mouse AlexaFluor647 (1:800, \#A-21236 Invitrogen, ThermoFisher Scientific), goat anti-rabbit AlexaFluor568 (1:800, \#A-11036 Invitrogen, ThermoFisher Scientific).

The hTERT-HPNE cells were fixed at 48h after transfection in formaldehyde 4\% (\#F1635-500ML, SigmaAldrich) in PBS for 15 min at RT, permeabilized with 1\% Triton X-100 in PBS and blocked with 2\% BSA in PBS for 20 min at RT. Incubation with primary antibody in $2 \%$ BSA/PBS was O.N. at $4^{\circ} \mathrm{C}$ and in secondary antibody plus DAPI (1:1000, Invitrogen, ThermoFisher Scientific) was $3 \mathrm{~h}$ at $4^{\circ} \mathrm{C}$. in $2 \% \mathrm{BSA} / \mathrm{PBS}$ for $3 \mathrm{~h}$. Human cells were washed once after fixation and permeabilization, and three times after each incubation with primary and secondary antibodies with PBS 10 minutes at RT. Fluorescence images were obtained at 40x magnification on a Leica DMI6000 FFW. Primary antibody used: anti-ARID1A (1:1000; \#HPA005456 Sigma-Aldrich). Secondary antibody used: donkey anti-rabbit (1:1000, \#A31573, ThermoFisher Scientific).

\section{Statistical Analysis}

Two-tailed paired Student's t-test from GraphPad Prism 5 (San Diego, CA, USA) was used. In all analyses, $\mathrm{P}<0.05$ was required for statistical significance.

In hTERT-HPNE immunohistochemistry images, ARID1A nuclear staining was measured for each cell and normalized for the average staining of the nucleus of all the cells in the same field (ratio=ARID1A expression/mean of ARID1A expression in the field). 


\section{II) Processing and Bioinformatic analysis}

\section{ChIP-seq analysis}

Raw reads for the two replicates of high quality (FASTQC v.0.11.5 $5^{40}$, Supplementary data 1 and 2) were aligned to the zebrafish genome (GRCz10/danRer10) using Bowtie2 (v.2.2.6) with default settings ${ }^{41}$. The file was converted into a bed file and the data extended $300 \mathrm{bp}$, bigwig tracks generated and uploaded to UCSC Genome Browser (Fig.1b). Highly enriched regions (peaks) were obtained by MACS14 (v.1.4.2) with the parameters "--nomodel, --nolambda and --space $=30$ " 42 . Reproducibility of the two biological replicates was measured by Pearson's correlation coefficient ${ }^{43}$.

\section{Analysis of enhancer activity}

To identify the best putative active enhancers, present in zebrafish adult pancreas, we intersected the peaks from the two H3K27ac replicates, selecting only the enriched regions present in both. Since H3K27ac is also present in promoter regions, we excluded peaks overlapping TSS coordinates. The genomic annotation of our putative enhancers was performed by HOMER annotatePeaks.pl ${ }^{44}$ (Fig. 1c). The adult pancreas putative active enhancer dataset (PsE+DevE) was crossed with the H3K27ac zebrafish embryonic dataset (dome, 80\% epiboly, $24 \mathrm{hpf}$ and $48 \mathrm{hpf}$ ) (SupplementaryTable15) ${ }^{33}$ to identify enriched regions present only in adult pancreas (PsE) (Fig.1d). All genomic intersections were performed using Bedtools "intersect"45. We superimposed the H3K27ac mapped reads from adult pancreas and the embryonic dataset with the adult pancreas H3K27ac peaks using seqMINER (v1.3.4) with default settings (Fig.1d), showing read densities \pm 5 $\mathrm{kb}$ from the acetylation peak center ${ }^{46}$. Gene enrichment and functional annotation of our dataset were obtained with GREAT $^{15}$, using the basal plus extension association rule (SupplementaryFig.3a).

\section{ATAC-seq analysis}

All libraries were sequenced on Illumina HiSeq 2500 platform and raw reads were mapped to the reference zebrafish genome (GRCz10/danRer10) using Bowtie2 (v.2.2.6) with parameters “-X 2000 and --verysensitive" ${ }^{\prime 4}$. To avoid clonal artefacts, the duplicated mapped reads were removed using Samtools ${ }^{47}$. Mapped reads were filtered by the fragment size $(\leq 120 \mathrm{bp})$ and mapping quality $(\geq 10)$. For a better visualization, data were extended $10 \mathrm{bp}$, generated bigwig tracks and uploaded to the UCSC browser (Fig.1b). To call for enriched regions, MACS2 (v.2.1.0) ${ }^{42}$ with the parameters “---nomodel, --keep-dup 1, --llocal 10000, --extsize 74, --shift - 37 and -p 0.07" were used. Reproducibility of the biological replicates was measured using the Pearson's correlation coefficient ${ }^{43}$. Then, we applied the Irreproducible Discovery Rate (IDR) in order to obtain a confident and reproducible set of peaks ${ }^{48}$. 


\section{C-seq analysis}

4C-seq libraries were first inspected for quality control using FASTQC (v.0.11.5 ${ }^{40}$, Supplementary data 35) and demultiplexed using the script "demultiplex.py" from the FourCSeq package ${ }^{49}$, allowing for 1 mismatch in the primer sequence. A custom perl script was used for subsequent processing, as previously described ${ }^{50,51}$. Reads were aligned to the zebrafish genome (GRCz10/danRer10) using Bowtie ${ }^{52}$, keeping only uniquely mapping reads (v1.1.2, -m 1). Reads within fragments flanked by restriction sites of the same enzyme or smaller than $40 \mathrm{bp}$ were filtered out. Mapped reads were then converted to reads-per-first-enzyme-fragmentend units, and smoothed using a 30 fragment mean running window algorithm (Fig. 4, 5).

\section{HiChIP-seq analysis}

HiChIP analysis from paired end fastq files to pairs of interacting fragments were performed using a custom python script based on the functions and defaults of the pytadbit python library ${ }^{53}$. This library first uses GEM mapper ${ }^{54}$ to map paired reads independently to the zebrafish reference genome (GRCz10/danRer10, flags used by GEM mapper --max-decoded-strata 1; --min-decoded-strata 0 ; -e 0.04). Then, reads are associated to a particular restriction fragment and paired together according to their read names. Once the reads are paired, the pairs of reads are filtered so that only those belonging to different restriction fragments are kept. Compressed sparse matrix files in cooler and hic formats were generated from those filtered reads using Cooler ("cload pairix" utility) and Juicer tools ("pre" utility) respectively for both visualization and further analysis. In order to predict the target promoter of putative enhancers a custom $\mathrm{R}$ script was designed, that uses uncompressed $5 \mathrm{~kb}$ resolution sparse matrices as inputs. Those matrices were obtained using Juicer tools ("dump" utility) from the hic file and filtered for $>=2$ interactions between 2 interacting chunks $<100 \mathrm{~kb}$ apart. In addition, only contacts connecting zebrafish pancreas active TSSs and putative active enhancers given by H3K27ac peaks from whole pancreas, adult pancreas (PsE), developing pancreas (DevE) and the different enhancer clusters (C1-C4) were included. This way, we could obtain the targets of putative active enhancers. An output table was produced with genes targeted by enhancers, per enhancer cluster (SupplementaryTable8). Custom scripts are provided in a gitlab repository (https://gitlab.com/rdacemel/pancreasregulome).

\section{Identification of active promoters}

H3K4me3 sequencing datasets (2 replicates performed in the HiChIP assay; Supplementary data 6-9) were aligned to the zebrafish genome (GRCz10/danRer10) using Bowtie2 (v.2.2.6) with default settings. Highly enriched regions (peaks) were obtained by MACS14 (v.1.4.2). algorithm with the parameters "--nomodel, -nolambda and --space $=30^{\prime \prime 4}$. Then, the peaks present in both replicates were filtered with the transcription start site (TSS) position to identify the active promoters using Bedtools "intersect" 45 


\section{RNA-seq analysis}

Total RNA extracted from adult zebrafish (exocrine, endocrine and muscle) and sequenced on Illumina HiSeq 2000 platform was inspected for quality control using FASTQC (v.0.11.5 ${ }^{40}$, Supplementary data 10-17). Then, sequences were trimmed to remove adaptors, sequencing artefacts and low-quality reads $(Q<20)^{55}$. The BWA-MEM software was used to map the clean reads to the reference genome (ZV9/danRer7) with the parameters "-w 2 and -c 3" ${ }^{\text {"56 }}$. Gene expression was measured from the mapped reads using HT-seq-count ${ }^{57}$. In addition, two public RNA-seq datasets were used (SupplementaryTable15).

\section{Gene expression barplots}

The average expression of genes associated with each enhancer cluster (PsE, DevE, C1-C4), as defined by HiChIP, was compared to the average expression of all genes present in the RNA-seq datasets using a custom R script and ggplot for drawing barplots (Fig.2a, SupplementaryFig.3b).

\section{Conservation between zebrafish and human and PhastCons scores}

To obtain the percentage of putative active enhancers conserved with human, the coordinates of putative active enhancers from adult zebrafish pancreas and embryos at different development stages (GRCz10/danRer10) were used as input to the UCSC genome coordinate conversion tool (https://genome.ucsc.edu/cgibin/hgLiftOver, liftover to hg19, October 2019) (Fig.3a). To visualize the conservation of the respective sequences, liftOver to hg38 was done and their average PhastCons conservation score plotted (Fig.3b). For this, we downloaded PhastCons scores in bigWig format from a 100-way multiple species alignment of vertebrates against human (hg38) (hg38.phastCons100way.bw, October 2019) ${ }^{58}$ and converted to BedGraph text format using the UCSC's utility bigWigToBedGraph. Then, the Bedtools ${ }^{45}$ suite (v.2.27) was used to intersect and map different putative enhancer clusters in bed format with the conservation scores, storing for each putative enhancer the median and average PhastCons score. To know which of them overlap putative active enhancers in human pancreas, we used the Bedtools "intersect" tool with default $\geq 1$ bp of overlap (Fig. $3 b$, blue). To check whether the conserved sequences showed a higher overlap with H3K27ac signal in humans than random, suggesting a higher likelihood of being also a putative active enhancer, we did the ratio of the number of conserved sequences overlapping H3K27ac signal in human over a similar one obtained from the average of a $10^{\wedge} 5$ random shuffling of the putative enhancer coordinates on the genome and their overlap with putative active enhancers in human (Fig. 3c).

\section{Transcription factor binding motifs enrichment}

To refine our data, H3K27ac peaks were filtered with the ATAC-seq peaks. Then, the transcription factor binding site (TFBS) predictor program Hypergeometric Optimization of Motif EnRichment (HOMER) was 
used to identify conserved sequence motifs enriched ${ }^{44}$. To evaluate our results, we also analysed, using HOMER, different acetylation data from: human pancreas, human ventricle, $24 \mathrm{hpf}$ and dome+80\%epiboly (SupplementaryTable14 and 15). From each sample, the top 140 enriched motifs were selected, and the groups compared by performing hypergeometric enrichment tests (Fig. 3G, SupplementaryFig.7a-e). Chi-square test from GraphPad Prism 7 (v.7.04) was performed to evaluate the enrichment in 25 known pancreas-related TFs. The HOMER software was also applied in PsE, C1, C2, C3 and C4 in order to identify the TFBS present (SupplementaryFig.7f, SupplementaryTable14).

\section{Identification of super-enhancers}

We applied ROSE (Ranking Ordering of Super-Enhancers) algorithm with default parameters to define superenhancers in our whole pancreas acetylation data and in human pancreas acetylation data ${ }^{59}$. Then, we performed gene ontology analysis in both data using PANTHER software (v.14.0, on April 2019) and compare the molecular functions obtained (http://pantherdb.org). To identify the genes shared between the two groups, we identified the human orthologous genes in our zebrafish list using Biomart (https://www.ensembl.org/biomart; on April 2019) and compared the groups (SupplementaryFig.6).

\section{Disease association enrichment of genes from different enhancer clusters}

Gene-disease association data were retrieved from DisGeNET (v.6.0) ${ }^{60}$ (http://www.disgenet.org/), Integrative Biomedical Informatics Group GRIB/IMIM/UPF, on April 2019, selecting for pancreas-related diseases and filtering for a minimum score of 0.1 to exclude associations only from text-mining and keeping only diseases associated to at least 10 genes. Gene annotations were obtained from Ensembl via BioMart on April 2019 (BioMart export file available upon request) selecting protein coding genes in zebrafish and gene homologs between human and zebrafish. For each pancreas-related disease, zebrafish homologs of the human diseaseassociated genes were obtained and for the diseases remaining with at least 15 genes, hypergeometric tests were performed for each cluster (PsE, DevE, C1-C4) taking the set of zebrafish protein coding genes as the population size, the number of disease genes as number of successes and the number of genes in a given cluster as the sample size. The R package "qvalue" was used to correct for multiple testing using FDR and convert unadjusted p-values into q-values ${ }^{61}$. Hypergeometric enrichment was obtained as the ratio "(number disease genes in clusterX / number of genes in clusterX) / (number disease genes / number of protein coding genes)". Finally, diseases with an absolute enrichment $\geq 1.5$ and a q-value $\leq 0.05$ were considered significantly enriched(/depleted) in the respective cluster (Fig.3d). 


\section{References}

1. Peery, A. F. et al. Burden and Cost of Gastrointestinal, Liver, and Pancreatic Diseases in the United States: Update 2018. Gastroenterology 156, 254-272.e11 (2019).

2. Zheng, Y., Ley, S. H. \& Hu, F. B. Global aetiology and epidemiology of type 2 diabetes mellitus and its complications. Nat Rev Endocrinol 14, 88-98 (2018).

3. Ong, C.-T. \& Corces, V. G. Enhancer function: new insights into the regulation of tissuespecific gene expression. Nat. Rev. Genet. 12, 283-293 (2011).

4. Furlong, E. E. M. \& Levine, M. Developmental enhancers and chromosome topology. Science 361, 1341 (2018).

5. Pasquali, L. et al. Pancreatic islet enhancer clusters enriched in type 2 diabetes risk-associated variants. Nat Genet 46, 136-143 (2014).

6. Thurner, M. et al. Integration of human pancreatic islet genomic data refines regulatory mechanisms at Type 2 Diabetes susceptibility loci. Elife 7, (2018).

7. Weedon, M. N. et al. Recessive mutations in a distal PTF1A enhancer cause isolated pancreatic agenesis. Nat Genet 46, 61-64 (2014).

8. Hwang, W. Y. et al. Efficient genome editing in zebrafish using a CRISPR-Cas system. Nat. Biotechnol. 31, 227-229 (2013).

9. Kinkel, M. D. \& Prince, V. E. On the diabetic menu: zebrafish as a model for pancreas development and function. Bioessays 31, 139-152 (2009).

10. Prince, V. E., Anderson, R. M. \& Dalgin, G. Zebrafish Pancreas Development and Regeneration: Fishing for Diabetes Therapies. Curr. Top. Dev. Biol. 124, 235-276 (2017).

11. Rada-Iglesias, A. et al. A unique chromatin signature uncovers early developmental enhancers in humans. Nature 470, 279-283 (2011).

12. Buenrostro, J. D., Giresi, P. G., Zaba, L. C., Chang, H. Y. \& Greenleaf, W. J. Transposition of native chromatin for fast and sensitive epigenomic profiling of open chromatin, DNA-binding proteins and nucleosome position. Nat. Methods 10, 1213-1218 (2013).

13. Mumbach, M. R. et al. HiChIP: efficient and sensitive analysis of protein-directed genome architecture. Nat. Methods 13, 919-922 (2016).

14. Guenther, M. G., Levine, S. S., Boyer, L. A., Jaenisch, R. \& Young, R. A. A chromatin landmark and transcription initiation at most promoters in human cells. Cell 130, 77-88 (2007).

15. Hiller, M. et al. Computational methods to detect conserved non-genic elements in phylogenetically isolated genomes: application to zebrafish. Nucleic Acids Res. 41, e151 (2013).

16. White, R. J. et al. A high-resolution mRNA expression time course of embryonic development in zebrafish. Elife 6, (2017).

17. Hinrichs, A. S. et al. The UCSC Genome Browser Database: update 2006. Nucleic Acids Res. 34, D590-598 (2006).

18. Jones, S. et al. Somatic Mutations in the Chromatin Remodeling Gene ARID1A Occur in Several Tumor Types. Hum Mutat 33, 100-103 (2012). 
19. Wu, J. N. \& Roberts, C. W. M. ARID1A mutations in cancer: another epigenetic tumor suppressor? Cancer Discov 3, 35-43 (2013).

20. Miguel-Escalada, I. et al. Human pancreatic islet three-dimensional chromatin architecture provides insights into the genetics of type 2 diabetes. Nature Genetics 51, 1137-1148 (2019).

21. Wang, S. C. et al. SWI/SNF component ARID1A restrains pancreatic neoplasia formation. Gut 68, 1259-1270 (2019).

22. Wang, W. et al. ARID1A, a SWI/SNF subunit, is critical to acinar cell homeostasis and regeneration and is a barrier to transformation and epithelial-mesenchymal transition in the pancreas. Gut 68, 1245-1258 (2019).

23. Pashos, E., Park, J. T., Leach, S. \& Fisher, S. Distinct enhancers of ptf1a mediate specification and expansion of ventral pancreas in zebrafish. Dev. Biol. 381, 471-481 (2013).

24. Klein, A. P. et al. Genome-wide meta-analysis identifies five new susceptibility loci for pancreatic cancer. Nat Commun 9, 556 (2018).

25. Wolpin, B. M. et al. Genome-wide association study identifies multiple susceptibility loci for pancreatic cancer. Nat. Genet. 46, 994-1000 (2014).

26. Mahajan, A. et al. Fine-mapping type 2 diabetes loci to single-variant resolution using highdensity imputation and islet-specific epigenome maps. Nat. Genet. 50, 1505-1513 (2018).

27. Morris, A. P. et al. Large-scale association analysis provides insights into the genetic architecture and pathophysiology of type 2 diabetes. Nat. Genet. 44, 981-990 (2012).

28. Khoueiry, P. et al. Uncoupling evolutionary changes in DNA sequence, transcription factor occupancy and enhancer activity. Elife 6, (2017).

29. Kimura, Y. et al. ARID1A Maintains Differentiation of Pancreatic Ductal Cells and Inhibits Development of Pancreatic Ductal Adenocarcinoma in Mice. Gastroenterology 155, 194-209.e2 (2018).

30. Jin, K. \& Xiang, M. Transcription factor Ptf1a in development, diseases and reprogramming. Cell. Mol. Life Sci. 76, 921-940 (2019).

31. Westerfield, M. The zebrafish book. A guide for the laboratory use of zebrafish (Danio rerio). (Univ. of Oregon Press).

32. Ishibashi, M., Mechaly, A. S., Becker, T. S. \& Rinkwitz, S. Using zebrafish transgenesis to test human genomic sequences for specific enhancer activity. Methods 62, 216-225 (2013).

33. Bogdanovic, O. et al. Dynamics of enhancer chromatin signatures mark the transition from pluripotency to cell specification during embryogenesis. Genome Res. 22, 2043-2053 (2012).

34. Fernández-Miñán, A., Bessa, J., Tena, J. J. \& Gómez-Skarmeta, J. L. Assay for transposaseaccessible chromatin and circularized chromosome conformation capture, two methods to explore the regulatory landscapes of genes in zebrafish. Methods Cell Biol. 135, 413-430 (2016).

35. de la Calle-Mustienes, E. et al. A functional survey of the enhancer activity of conserved noncoding sequences from vertebrate Iroquois cluster gene deserts. Genome Res. 15, 1061-1072 (2005).

36. Bessa, J. et al. Zebrafish enhancer detection (ZED) vector: a new tool to facilitate transgenesis and the functional analysis of cis-regulatory regions in zebrafish. Dev. Dyn. 238, 2409-2417 (2009). 
37. Bessa, J. et al. A mobile insulator system to detect and disrupt cis-regulatory landscapes in vertebrates. Genome Res. 24, 487-495 (2014).

38. Kawakami, K. et al. A transposon-mediated gene trap approach identifies developmentally regulated genes in zebrafish. Dev. Cell 7, 133-144 (2004).

39. Moreno-Mateos, M. A. et al. CRISPRscan: designing highly efficient sgRNAs for CRISPRCas9 targeting in vivo. Nat Methods 12, 982-988 (2015).

40. Andrews, S. FastQC: a quality control tool for high throughput sequence data. http://www.bioinformatics.babraham.ac.uk/projects/fastqc.

41. Langmead, B. \& Salzberg, S. L. Fast gapped-read alignment with Bowtie 2. Nat. Methods 9, 357-359 (2012).

42. Zhang, Y. et al. Model-based analysis of ChIP-Seq (MACS). Genome Biol. 9, R137 (2008).

43. Bailey, T. et al. Practical guidelines for the comprehensive analysis of ChIP-seq data. PLoS Comput. Biol. 9, e1003326 (2013).

44. Heinz, S. et al. Simple combinations of lineage-determining transcription factors prime cisregulatory elements required for macrophage and B cell identities. Mol Cell 38, 576-589 (2010).

45. Quinlan, A. R. \& Hall, I. M. BEDTools: a flexible suite of utilities for comparing genomic features. Bioinformatics 26, 841-842 (2010).

46. Zhan, X. \& Liu, D. J. SEQMINER: An R-Package to Facilitate the Functional Interpretation of Sequence-Based Associations. Genet. Epidemiol. 39, 619-623 (2015).

47. Li, H. et al. The Sequence Alignment/Map format and SAMtools. Bioinformatics 25, 20782079 (2009).

48. Li, Q., Brown, J. B., Huang, H. \& Bickel, P. J. Measuring reproducibility of high-throughput experiments. Ann. Appl. Stat. 5, 1752-1779 (2011).

49. Klein, F. A. et al. FourCSeq: analysis of 4C sequencing data. Bioinformatics 31, 3085-3091 (2015).

50. Noordermeer, D. et al. The dynamic architecture of Hox gene clusters. Science 334, 222-225 (2011).

51. Splinter, E., de Wit, E., van de Werken, H. J. G., Klous, P. \& de Laat, W. Determining longrange chromatin interactions for selected genomic sites using 4C-seq technology: from fixation to computation. Methods 58, 221-230 (2012).

52. Emera, D., Yin, J., Reilly, S. K., Gockley, J. \& Noonan, J. P. Origin and evolution of developmental enhancers in the mammalian neocortex. Proc Natl Acad Sci USA 113, E2617 (2016).

53. Serra, F. et al. Automatic analysis and 3D-modelling of Hi-C data using TADbit reveals structural features of the fly chromatin colors. PLoS Comput. Biol. 13, e1005665 (2017).

54. Marco-Sola, S., Sammeth, M., Guigó, R. \& Ribeca, P. The GEM mapper: fast, accurate and versatile alignment by filtration. Nat. Methods 9, 1185-1188 (2012).

55. Gordon A, Hannon G. Fastx-Toolkit. Fastq/a Short-Reads Pre-Processing Tools. Unpublished work (2003). 
56. Li, H. \& Durbin, R. Fast and accurate short read alignment with Burrows-Wheeler transform. Bioinformatics 25, 1754-1760 (2009).

57. Anders, S., Pyl, P. T. \& Huber, W. HTSeq--a Python framework to work with high-throughput sequencing data. Bioinformatics 31, 166-169 (2015).

58. Siepel, A. et al. Evolutionarily conserved elements in vertebrate, insect, worm, and yeast genomes. Genome Res. 15, 1034-1050 (2005).

59. Whyte, W. A. et al. Master transcription factors and mediator establish super-enhancers at key cell identity genes. Cell 153, 307-319 (2013).

60. Piñero, J. et al. DisGeNET: a discovery platform for the dynamical exploration of human diseases and their genes. Database (Oxford) 2015, bav028 (2015).

61. MacDonald, P. W., Liang, K. \& Janssen, A. Dynamic adaptive procedures that control the false discovery rate. Electron. J. Statist. 13, 3009-3024 (2019).

62. Tarifeño-Saldivia, E. et al. Transcriptome analysis of pancreatic cells across distant species highlights novel important regulator genes. BMC Biol. 15, 21 (2017). 


\section{Figure 1}
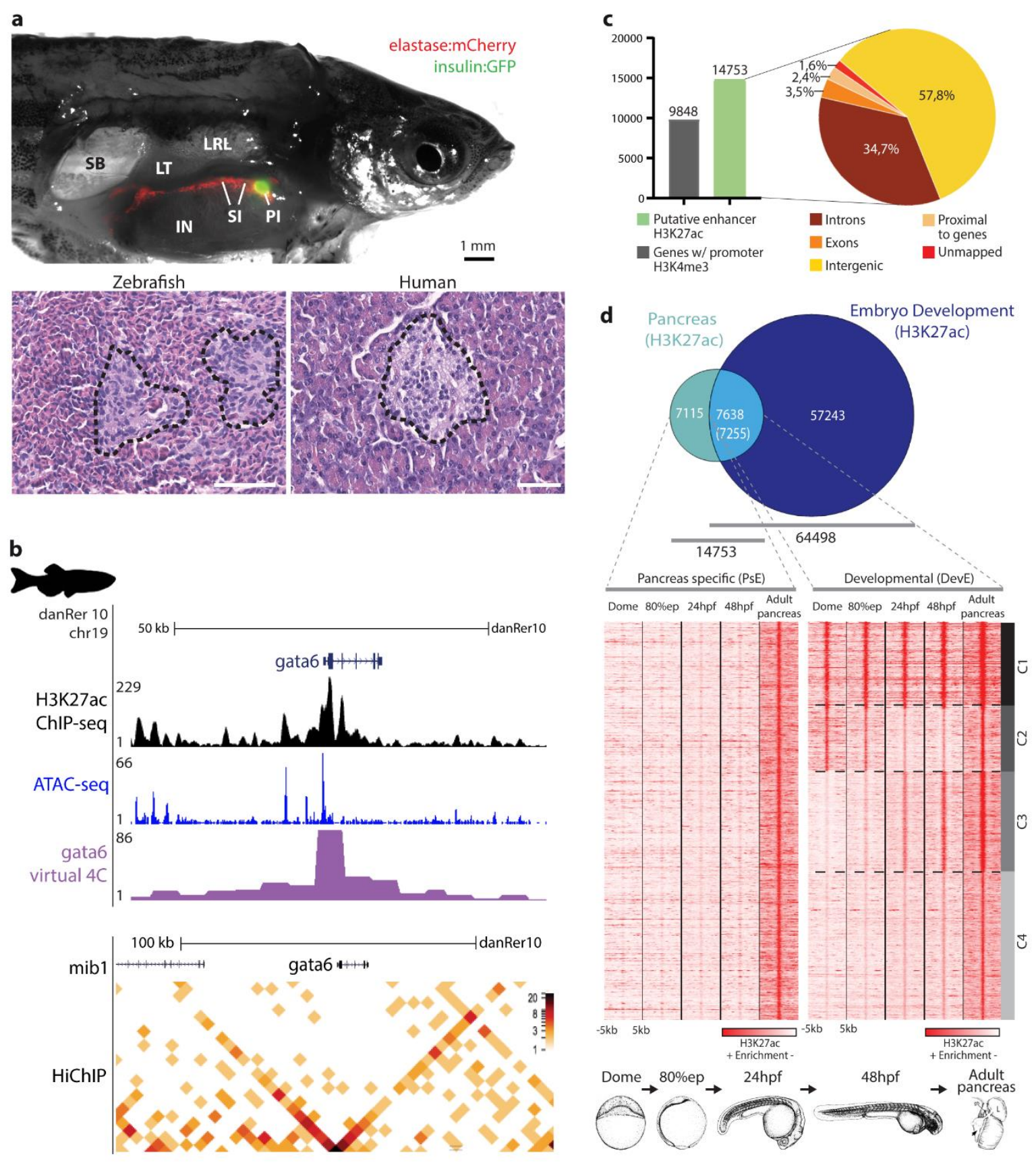

Figure 1. The zebrafish pancreas description, from histology to chromatin state. a) Comparison of adult zebrafish and human pancreas. Dissected adult male $\operatorname{Tg}($ insulin:GFP, elastase:mCherry) zebrafish (top); insulin and elastase promoters drive GFP expression in beta-cells (green) and mCherry in acinar cells (red), respectively. IN, intestine; LRL, Liver right lobe; LT, left testis; PI, principal islet; SI, secondary islets; SB, swim bladder. Histology of the pancreas, transverse sections with hematoxylin/eosin staining (bottom). Islets of Langerhans (black dashed lines) surrounded by exocrine tissue in zebrafish (left) and human (right), magnification 40x. b) Genomic landscape of 
gata6 in zebrafish pancreas with H3K27ac ChIP-seq profile (black), ATAC-seq peaks (blue) and gata6 virtual 4C (purple) based on the interactions with gata6 promoter detected by HiChIP for H3K4me3 (lower panel). c) Barplot (left) showing the number of genes with active promoters defined with H3K4me3 signal (gray bar) and putative active enhancers in adult zebrafish pancreas defined by H3K27ac mark (green bar), and their distribution throughout the regions of the genome (right). d) Venn diagram showing the overlap of putative enhancers in adult zebrafish pancreas and zebrafish embryonic development stages (top). Heat maps showing clusters of H3K27ac mark in dome, 80\% epiboly (80\%epi), 24hpf, 48hpf and adult pancreas for pancreas-specific enhancers (PsE) and developmental shared enhancers (DevE). A window of $10 \mathrm{~kb}$ around the reference coordinates for each sequence was used and the density files were subjected to k-means clustering, obtaining four different clusters in DevE: C1 - Cluster 1, C2 - Cluster 2, C3 - Cluster 3 and C4 - Cluster 4. 


\section{Figure 2}
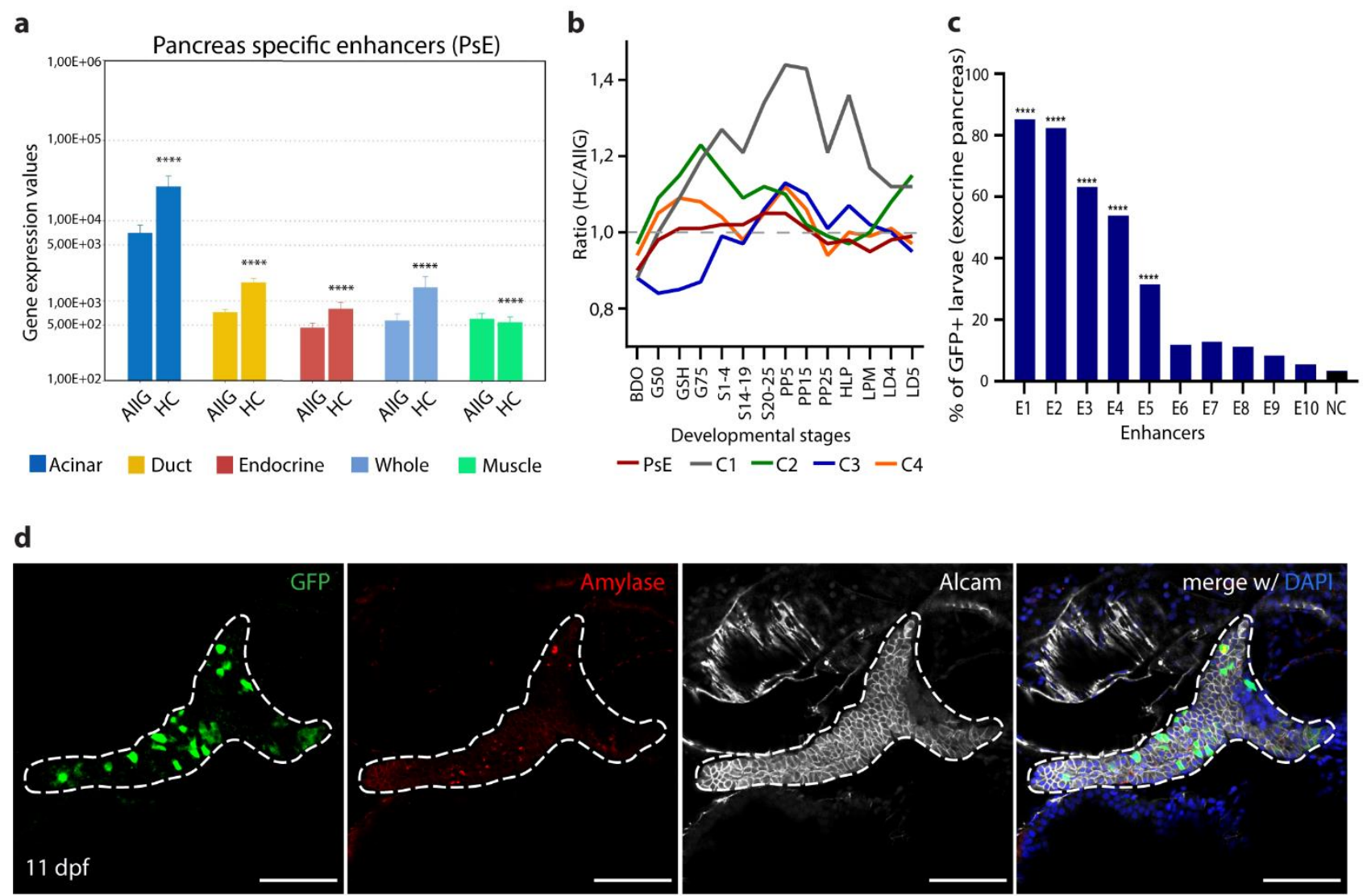

pancreatic domain

Figure 2. ChIP-seq and ATAC-seq data accurately predict functional pancreatic enhancers. a) Average expression of genes interacting with pancreas-specific enhancers detected by HiChIP for $\mathrm{H} 3 \mathrm{~K} 4 \mathrm{me} 3$ (HC), compared to the average expression of all genes (AllG). Gene expression was determined from RNA-seq data from different pancreatic cells (acinar, duct ${ }^{62}$, endocrine and whole pancreas) and muscle (control). Wilcoxon test, $p$-values $\leq 0.05$ were considered significant. **** $p<0,0001$. b) Ratio between the average expression of genes interacting with pancreas-specific enhancers (PsE, C1, C2, C3 and C4 clusters) and the average expression of all genes throughout zebrafish development. BDO: blastula, dome; G50: gastrula, 50\%epiboly; GSH: gastrula,shield; G75: gastrula,75\%epiboly; S1-4: segmentation, 1-4somites; S14-19: segmentation, 14-19somites; S20-25: segmentation, 20-25somites; PP5: pharyngula, prim5; PP15: pharyngula, prim15; PP25: pharyngula, prim25; HLP: hatching, long pec; LPM: larval, protruding mouth; LD4: larval, day4; LD5: larval, day5. c) Percentage of zebrafish larvae showing GFP expression in the pancreas after in vivo transient transgenesis reporter assays with Z48 vector. A total of 10 putative enhancer sequences (E1 to 10) were tested, using the empty enhancer reporter vector as negative control (NC). Values are represented as percentages and compared by Chi-square test. p-values $<0.05$ were considered significant $(* * * * \mathrm{p}<0.0001)$. d) Representative confocal image of the in vivo transient transgenesis reporter assays for the E3 sequence (zPtflaE1) showing expression of GFP (green) in 11dpf zebrafish pancreas 
bioRxiv preprint doi: https://doi.org/10.1101/2020.04.27.064220; this version posted April 28, 2020. The copyright holder for this preprint (which was not certified by peer review) is the author/funder. All rights reserved. No reuse allowed without permission.

(white dashed line), labelled by anti-Alcam staining (white) and anti-Amylase (red) antibodies. Nuclei were stained with DAPI (blue). Images were captured with a Leica SP5II confocal microscope. Scale bar $60 \mu \mathrm{m}$. 


\section{Figure 3}

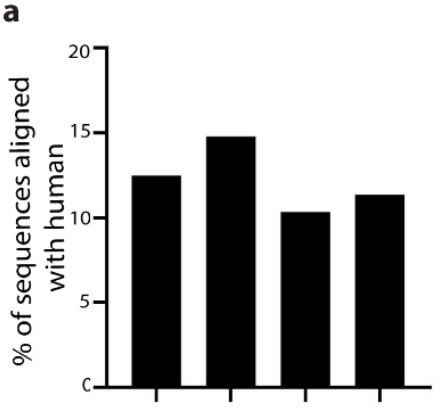

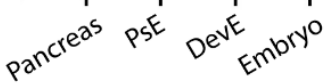

b

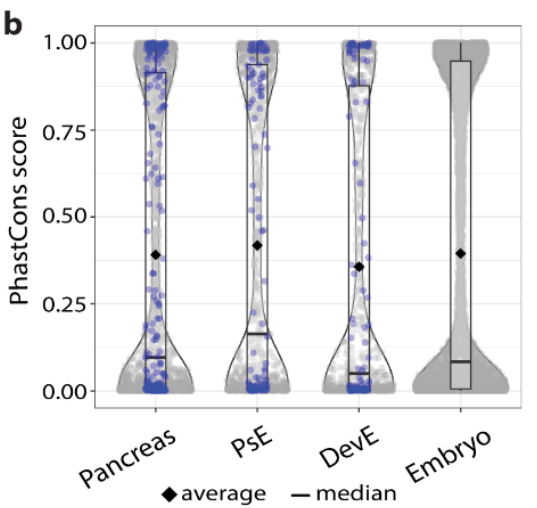

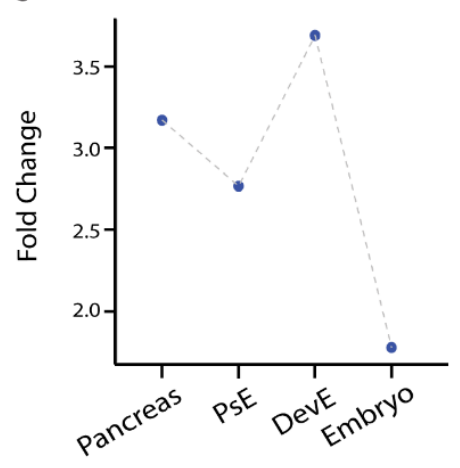

d

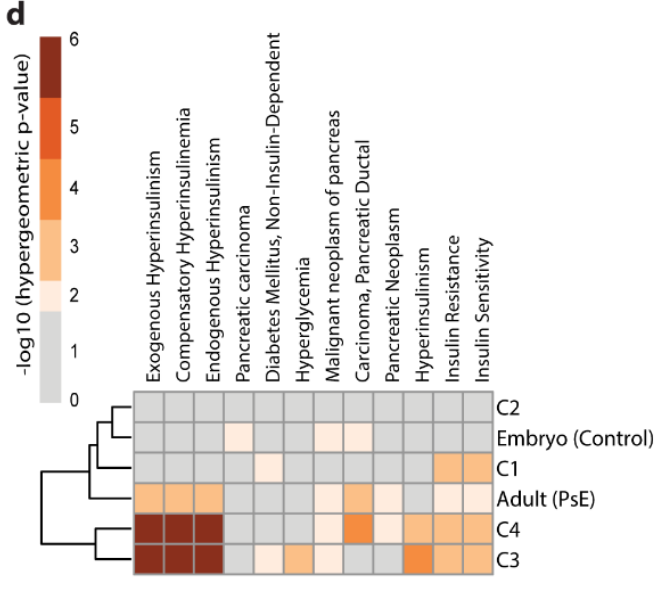

$\mathbf{f}$

GATA6

\section{ICTTATCTC도}

FOXA2

CTTCTTTACATA

CEBP
ATTGCCCAAC
HNF4a

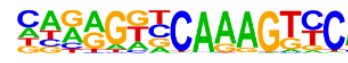

FOXA1

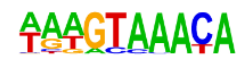

HNF1b e
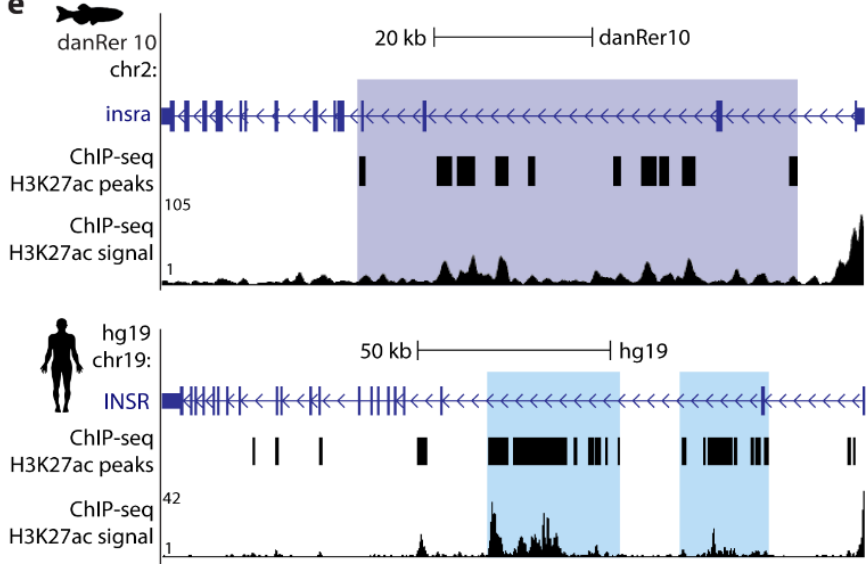

g

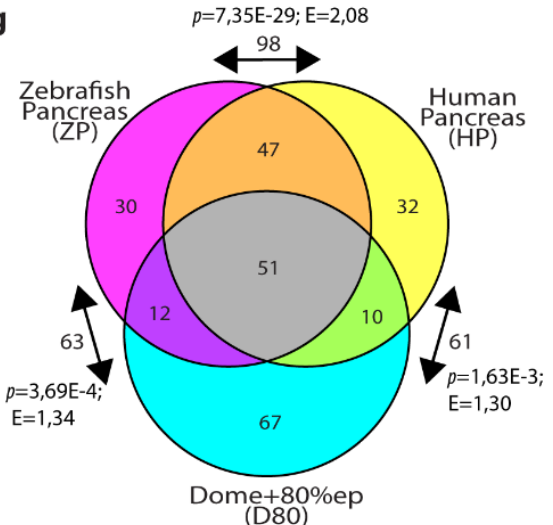

Figure 3. The zebrafish and human pancreas share cis-regulatory similarities. a) Percentage of predicted zebrafish pancreatic enhancer sequences that can be aligned to the human genome. Sequences are grouped in different clusters: putative enhancer sequences active in total pancreas (Pancreas), active only in differentiated adult pancreas (PsE), active in differentiated adult pancreas and during embryonic development (DevE) and active only during embryonic development (Embryo). b) PhastCon scores (99 vertebrate genomes) for human sequences aligned to zebrafish putative enhancers (Pancreas, PsE, DevE and Embryo; grey). In blue are depicted sequences that also show H3K27ac signal in human pancreas (data from ENCODE). c) Fold change of the number of zebrafish pancreatic $\mathrm{H} 3 \mathrm{~K} 27 \mathrm{ac}$ positive sequences that overlap with human pancreatic $\mathrm{H} 3 \mathrm{~K} 27 \mathrm{ac}$ 
positive sequences (ENCODE data), comparing to a $10^{\wedge} 5$ random shuffle of the used human sequences. d) Heat map showing - $\log _{10}$ (p-values) from hypergeometric tests of the enrichment for pancreatic disease association on the genes linked to each enhancer cluster identified by HiChIP for $\mathrm{H} 3 \mathrm{~K} 4 \mathrm{me} 3$. The coloured values meet the established criteria of statistical significance (q-value $\leq 0.05$ ) and fold change $(\operatorname{abs}(\mathrm{FC}) \geq 1.5)$. e) Genomic landscape of the human INSR gene (top) and zebrafish aridlab ortholog (bottom), showing H3K27ac signal and the position of predicted super-enhancers highlighted in blue. f) List of known relevant pancreas transcription factors (TFs) whose motifs are enriched in zebrafish pancreas H3K27ac ChIP-seq data. g) Venn diagram of the top 140 enriched TFBS motifs detected in H3K27ac positive sequences in three different datasets: zebrafish pancreas (ZP), human pancreas (HP) and dome+80\%epiboly embryos (D80). Arrows show the number of motifs shared between pairs of groups. Statistical significance was determined by hypergeometric enrichment test, $p$-values and the enrichment of the observed $v s$ expected are represented. 


\section{Figure 4}

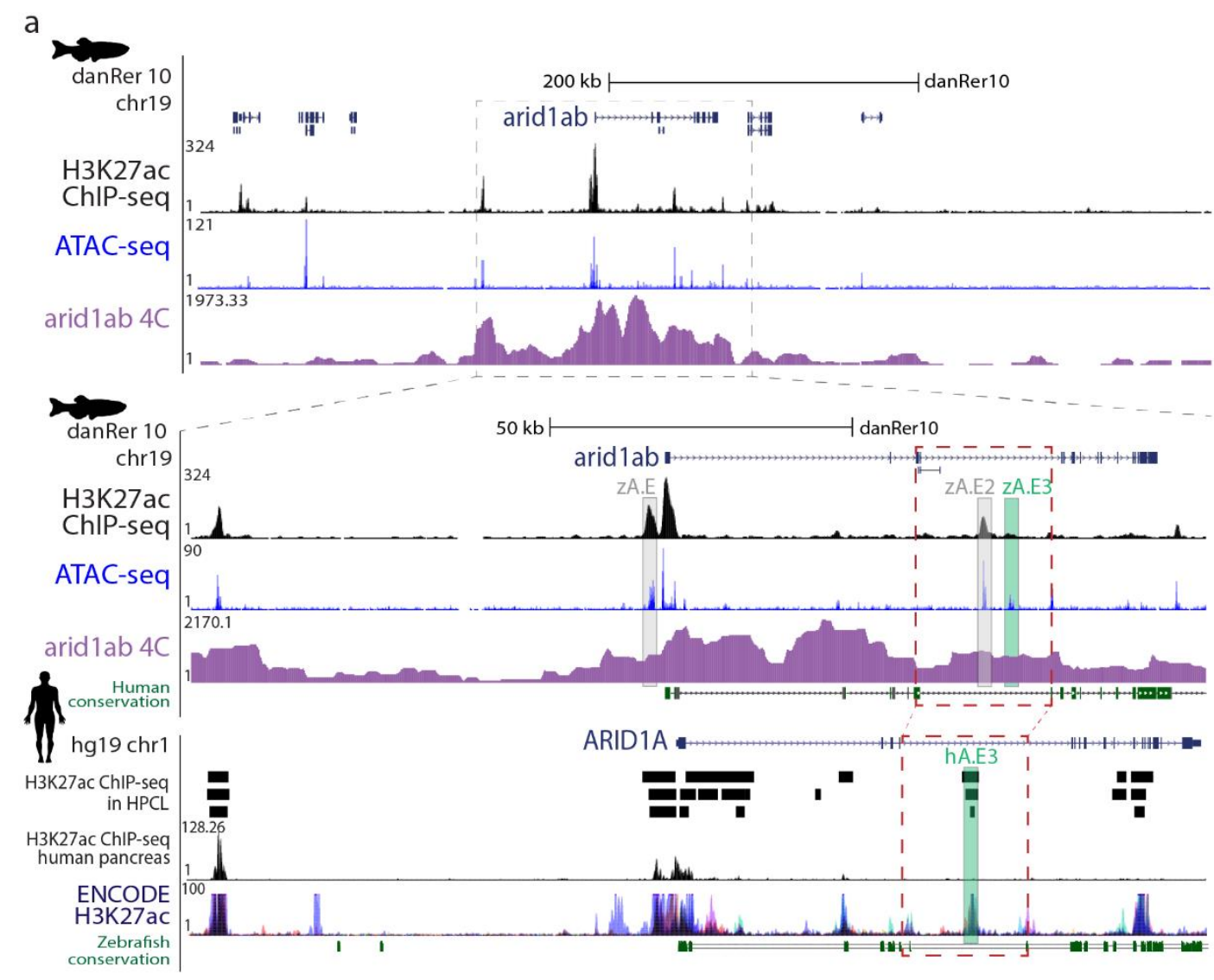

b
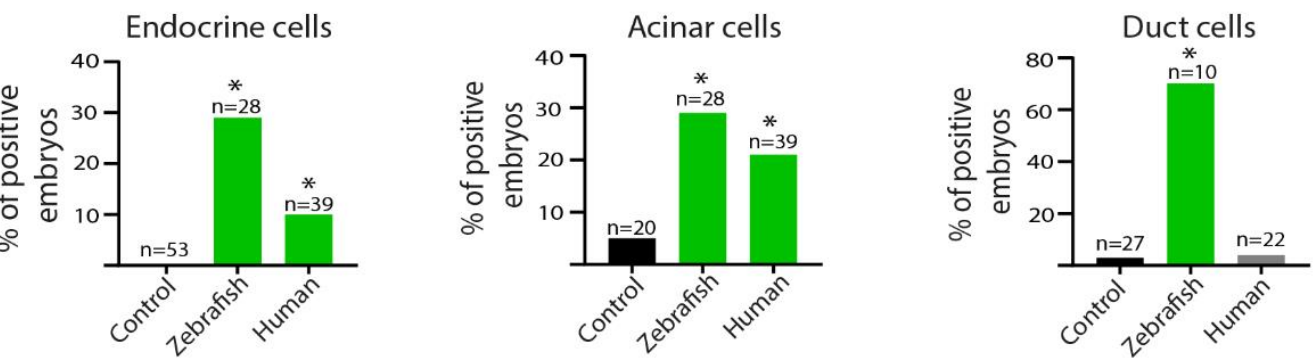

C
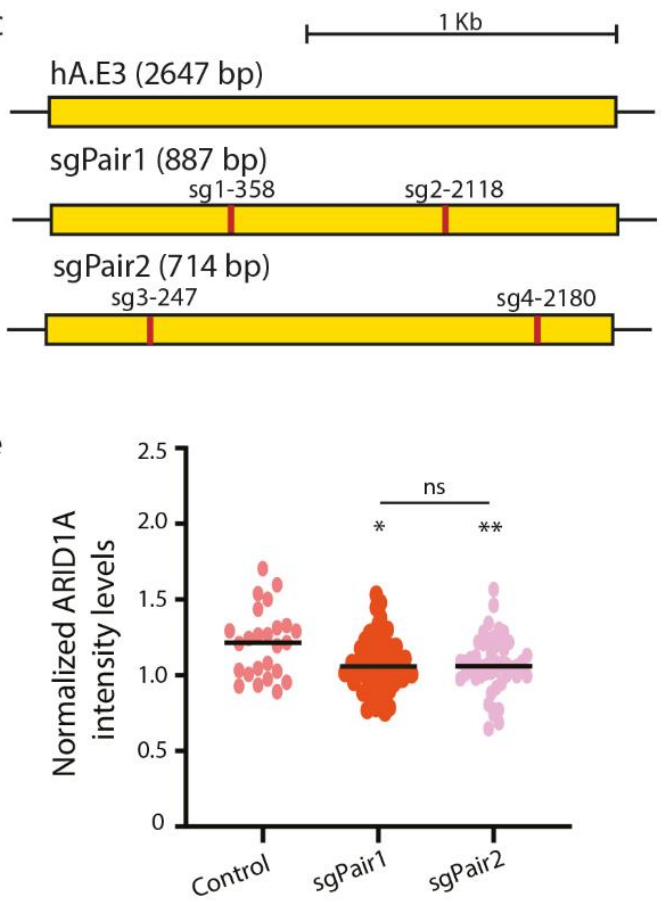

d
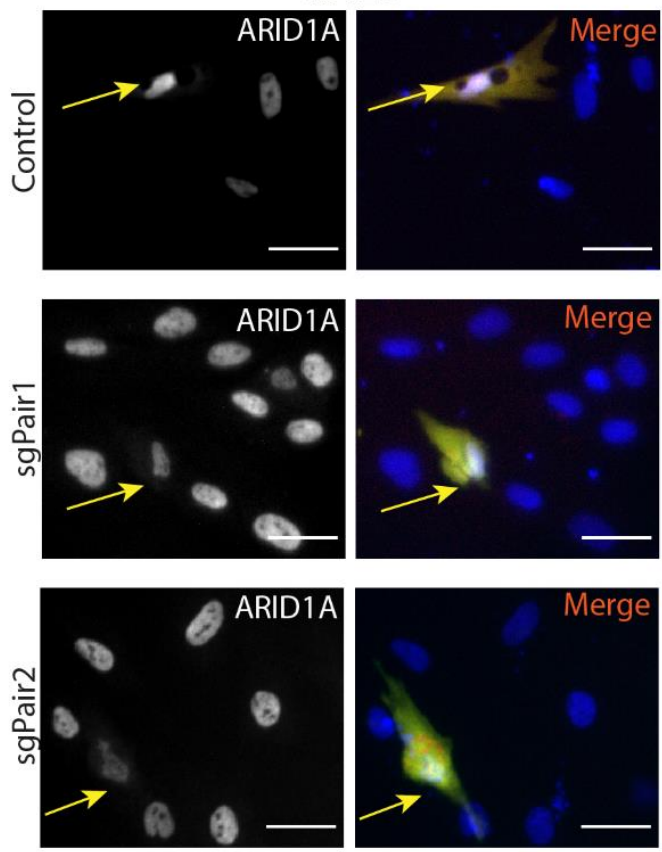
Figure 4. The zebrafish and human aridlab/ARIDIA regulatory landscapes contain an equivalent pancreatic enhancer. a) Genomic landscape of the zebrafish aridlab gene, showing profiles for H3K27ac ChIP-seq (black), ATAC-seq (blue) and 4C with view point in the aridlab promoter (purple) in adult zebrafish pancreas (upper panel); zoom-in of the aridlab gene and putative enhancers in its vicinity (middle panel). Genomic landscape of human ARIDIA (lower panel), with annotated $\mathrm{H} 3 \mathrm{~K} 27 \mathrm{ac}$ enriched intervals based on peak calling from different human pancreatic cell lines (HPCL, black bars), PT-45-P1, CFPAC-1 and HPAF-II, top to bottom, respectively, H3K27ac profile from human whole pancreatic tissue (WPT, black) and $\mathrm{H} 3 \mathrm{~K} 27 \mathrm{ac}$ profile from non-pancreatic human cell lines (NPHCL; GM12878, H1-hESC, HSMM, HUVEC, K562, NHEK and NHLF; Data from ENCODE). Sequence conservation between human and zebrafish is represented in dark green. Tested putative enhancers from zebrafish and human genomic DNA are highlighted in grey zA.E1 (zAridE1) and zA.E2 (zAridE2) and green zA.E3 (zAridE3) and hA.E1 (hAridE3). A zebrafish/human syntenic box containing the zAridE3 sequence and its putative equivalent human enhancer hAridE3 is highlighted (red dashed box). b) Percentage of zebrafish embryos showing GFP zAridE3 or hAridE3 mediated expression from transient transgenesis assays in endocrine, acinar and duct cells, at 11dpf. Statistical significance determined by Chi-square test with Yates correction $(* \mathrm{p}<0.05)$. c) Schematic representation of the targeting strategy for the hAridE3 locus, indicating the CRISPR sgRNA target sites and expected genomic deletions. d) Representative images of transfected hTERT-HPNE human cells assays. In the control, a region without H3K27ac ChIP-seq signal was deleted. In sgPair1 and sgPair2, the hAridE3 (hA.E3) locus was deleted using the respective pairs of sgRNAs. The double transfected cells are defined by GFP (green) and mCherry (red) co-expression and the nuclei were stained with DAPI (blue) and anti-ARID1A (grey). The yellow arrow is pointing to the double transfected cells. Images were captured with Leica DMI6000 FFW microscope. Scale bar: $40 \mu \mathrm{m}$. e) Normalized ARID1A intensity levels, measured from immunocytochemistry images, in control cells and in sgPair1 and sgPair2, where hA.E3 locus was deleted. Statistical significance is depicted in the graphs for $\mathrm{p} \leq 0.05, \mathrm{p} \leq 0.01\left(^{* *}\right)$ and $\mathrm{ns}$ for no statistical significance. These results were obtained from three batches of independent experiments. 
bioRxiv preprint doi: https://doi.org/10.1101/2020.04.27.064220; this version posted April 28, 2020. The copyright holder for this preprint (which was not certified by peer review) is the author/funder. All rights reserved. No reuse allowed without permission.

\section{Figure 5}

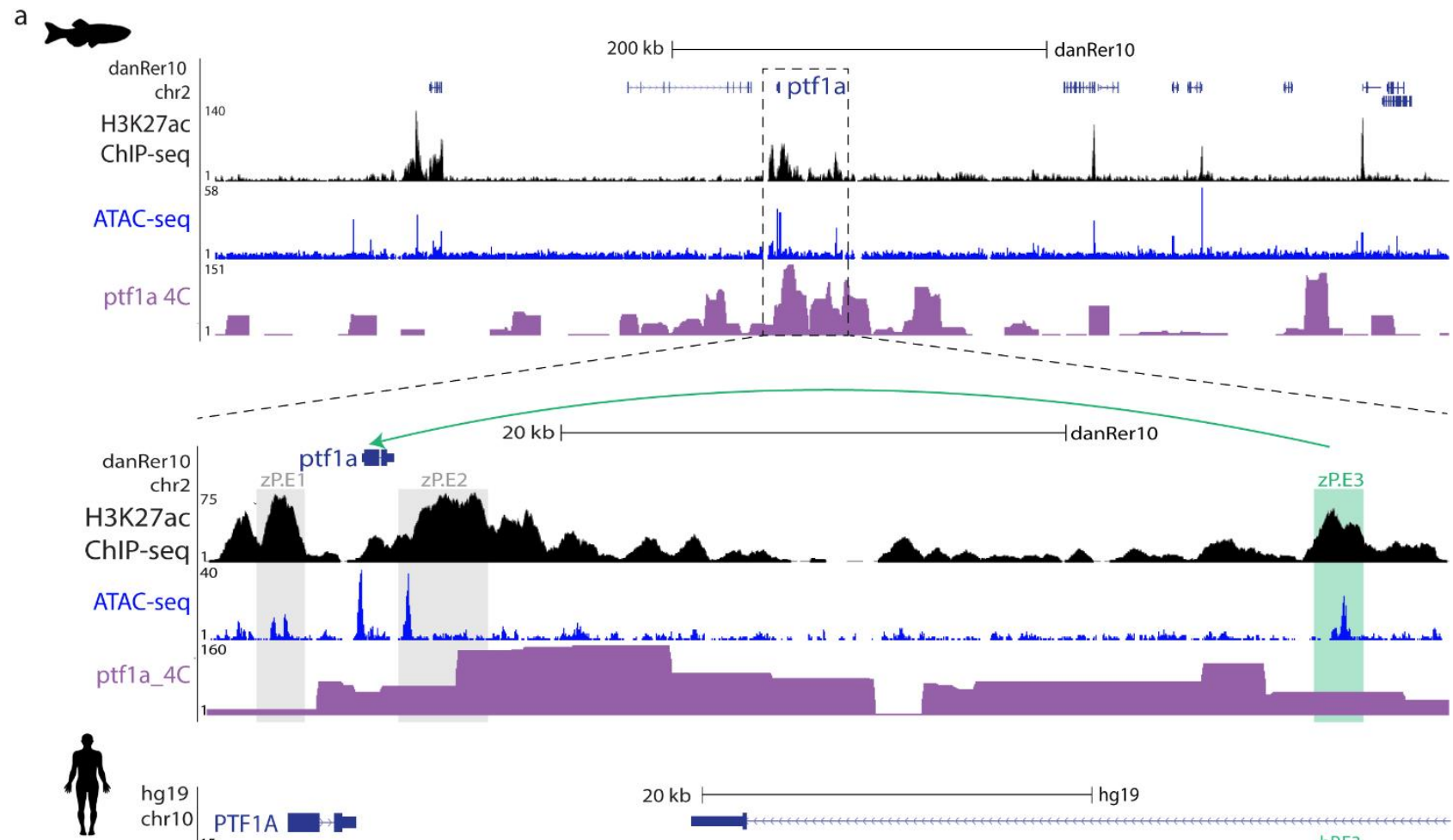

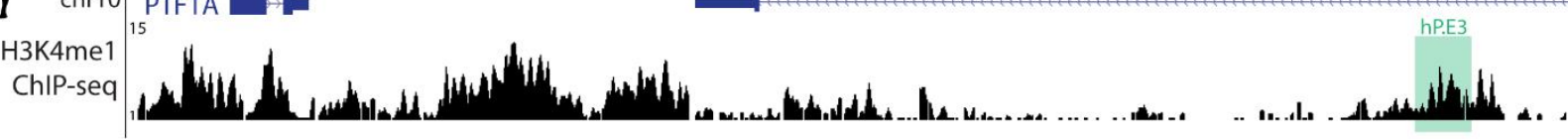

b

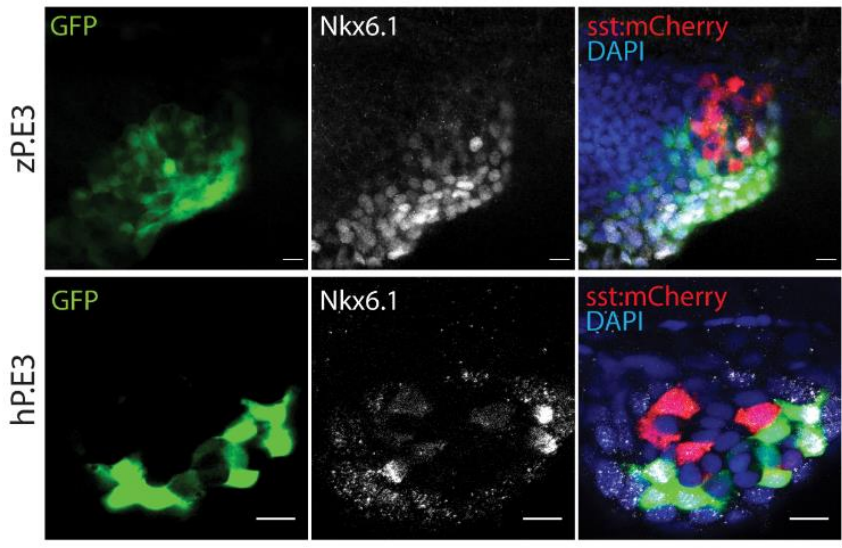

C

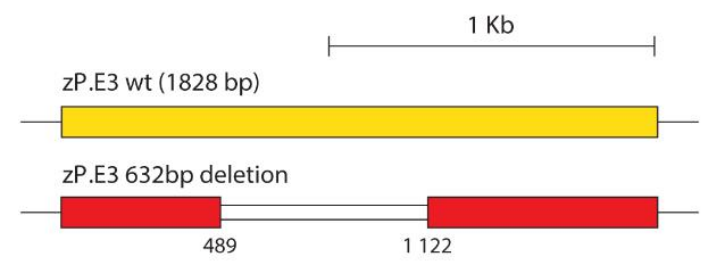

d
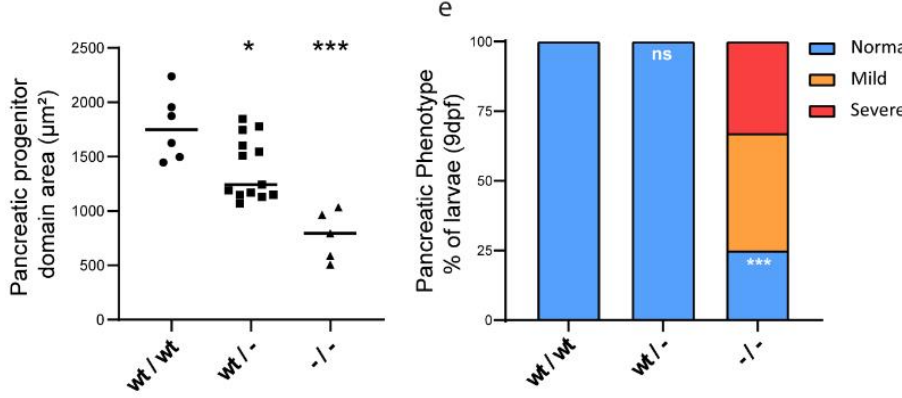
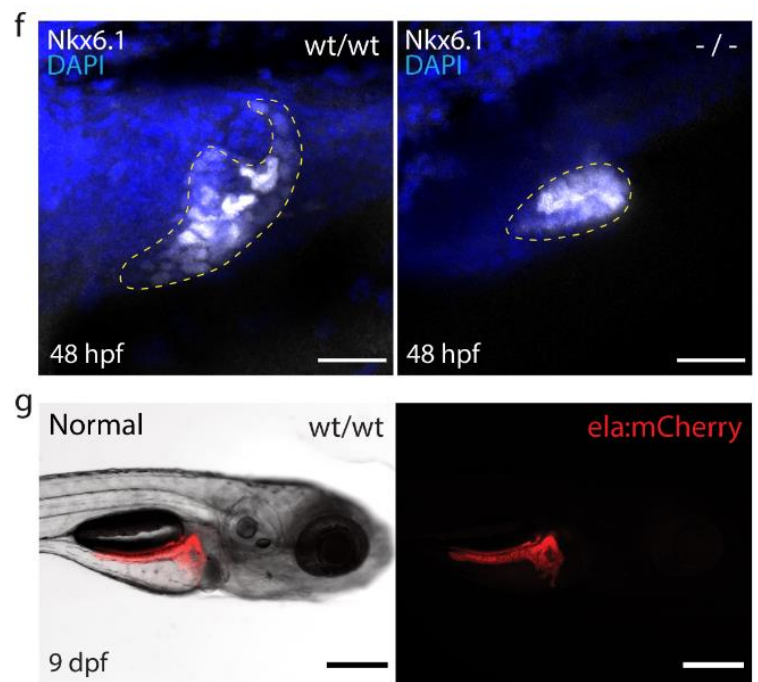

$9 \mathrm{dpf}$

Mild
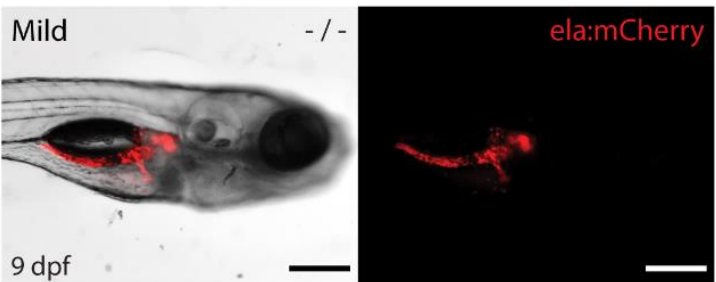

$9 \mathrm{dpf}$

Severe

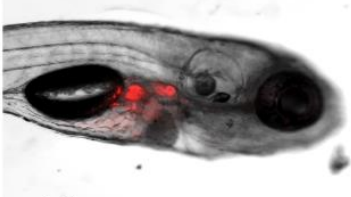

$9 \mathrm{dpf}$
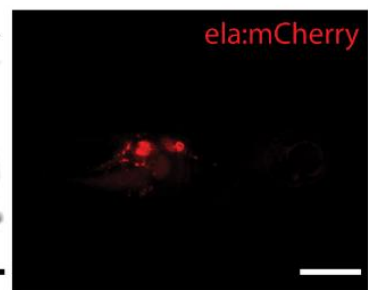
Figure 5. The Ptfla regulatory landscape in zebrafish and human contains a functional equivalent enhancer essential for pancreas formation. a) UCSC Genome Browser view of the zebrafish ptfla and human PTF1A genomic landscapes showing H3K27ac ChIP-seq (black), ATAC-seq (blue) and ptfla 4C interactions (purple) from whole zebrafish pancreas samples (upper panel), with a zoom-in (middle panel), and H3K4me1 ChIP-seq data (black) from human embryonic pancreatic progenitors (lower panel). Grey boxes highlight two previously validated zebrafish enhancers, zP.E1 (zptf1aE1) and zP.E2 (zptf1aE2) in the vicinity of the ptfla gene. Green boxes highlight a newly discovered distal enhancer in zebrafish (zP.E3 (zptf1aE3), and the location of its putative human functional ortholog (hP.E3). b) Confocal images of zebrafish reporter transgenic lines Tg(zP.E3:GFP) and Tg(hP.E3:GFP), showing co-localization of GFP expression (green) with anti-NKX6.1 (white), a marker of pancreatic progenitors, at $48 \mathrm{hpf}$. Delta-cells of the endocrine pancreas express mCherry (red) and nuclei are labelled with DAPI (blue). Scale bar $25 \mu \mathrm{m}$. c) Schematic depiction of the CRISPR/Cas9 mediated 632 bp deletion of the zP.E3 enhancer. d) Pancreatic progenitor domain area, defined by anti-NKX6.1 (white), of homozygous (-/-), heterozygous (wt/-) and wild type (wt/wt) embryos for the deletion of zP.E, at $48 \mathrm{hpf}$, (n=24). e) Percentage of larvae, -/-, wt/- and wt/wt, with different pancreatic phenotypic defects (normal, mild and severe) at $9 \mathrm{dpf},(\mathrm{n}=47)$. Fisher's exact test, p-values $<0.05$ were considered significant $\left(* * * p<0.001,{ }^{*} p<0.05\right)$. f) Representative confocal images (maximum intensity projections) of the pancreatic progenitor domain (NKX6.1, white) of zP.E3wt/wt and zP.E3-/- embryos at 48 hpf. Nuclei are stained with DAPI. Scale bar: $25 \mu \mathrm{m}$. g) Epifluorescence live images of representative phenotypes quantified in e), for $\operatorname{Tg}($ elastase:mCherry) zebrafish larvae at $9 \mathrm{dpf}$. Scale bar: $250 \mu \mathrm{m}$. 


\section{Table of contents}

List of supplementary figures:

Supplementary figure 1 - page 3

Supplementary figure 2 - page 4

Supplementary figure 3 - page 5

Supplementary figure 4 - page 7

Supplementary figure 5 - page 8

Supplementary figure 6 - page 10

Supplementary figure 7 - page 12

Supplementary figure 8 - page 14

Supplementary figure 9 - page 16

Supplementary figure 10 - page 17

Supplementary figure 11 - page 19

Supplementary figure 12 - page 21

Supplementary figure 13 - page 22

Supplementary figure 14 - page 23

Supplementary figure 15 - page 25

Supplementary figure 16 - page 27

Supplementary figure 17 - page 29

List of supplementary tables:

Supplementary table 1 - Genomic coordinates of pancreatic enhancers (excel file)

Supplementary table 2 - List of genes (excel file)

Supplementary table 3 - List of active promoters captured by HiChIP (H3K4me3) (excel file)

Supplementary table 4 - List of putative enhancers (PsE, DevE, Embryo) (excel file)

Supplementary table 5 - List of putative enhancers (C1-C4) (excel file)

Supplementary table 6 - Enrichment of the expression of genes associated by GREAT to PsE (Output from GREAT) (excel file)

Supplementary table 7 - Enrichment of the expression of genes associated by GREAT to DevE (Output from GREAT) (excel file) 
Supplementary table 8 - HiChIP results: number of interactions between a $5 \mathrm{~kb}$ bin containing a putative active enhancer in whole pancreas and a $5 \mathrm{~kb}$ bin containing the TSS of the indicated gene (excel file)

Supplementary table 9 - Zebrafish tissue RNA-seq raw counts (excel file)

Supplementary table 10 - List of putative pancreatic enhancer sequences tested (excel file)

Supplementary table 11 - Fig3C stats (100k times shuffling hg38 coordinates lifted over from zebrafish putative active enhancer sets and checking their intersect with human pancreas H3K27ac peaks) (excel file)

Supplementary table 12 - Hypergeometric test enrichment for the association to pancreas-related diseases on the genes from each enhancer cluster (Embryo, C1-C4, PsE) (excel file)

Supplementary table $\mathbf{1 3}$ - Super-enhancers active in zebrafish pancreas predicted by ROSE (excel file)

Supplementary table 14 - List of TF found by HOMER (excel file)

Supplementary table 15 - List of data used (excel file)

Supplementary table 16 - List of primers used (excel file)

\section{List of supplementary data:}

Supplementary data 1 - Pancreas H3K27ac ChIP-seq fastqc 1 (html file)

Supplementary data 2 - Pancreas H3K27ac ChIP-seq fastqc 2 (html file)

Supplementary data 3-4C-seq Arid1ab fastqc 1 (html file)

Supplementary data 4 -4C-seq Arid1ab fastqc 2 (html file)

Supplementary data 5 - 4C-seq Ptf1a fastqc (html file)

Supplementary data 6- Pancreas H3K4me3 HiChIP fastqc 1-1 (html file)

Supplementary data 7- Pancreas H3K4me3 HiChIP fastqc 1-2 (html file)

Supplementary data 8- Pancreas H3K4me3 HiChIP fastqc 2-1 (html file)

Supplementary data 9- Pancreas H3K4me3 HiChIP fastqc 2-2 (html file)

Supplementary data 10-RNA-seq Endocrine old fastqc 1 (html file)

Supplementary data 11-RNA-seq Endocrine old fastqc 2 (html file)

Supplementary data 12-RNA-seq Endocrine young fastqc 1 (html file)

Supplementary data 13-RNA-seq Endocrine young fastqc 2 (html file)

Supplementary data 14- RNA-seq Exocrine old fastqc (html file)

Supplementary data 15- RNA-seq Exocrine young fastqc (html file)

Supplementary data 16- RNA-seq Muscle young fastqc (html file)

Supplementary data 17- RNA-seq Muscle old fastqc (html file) 


\section{Supplementary figure 1}

a
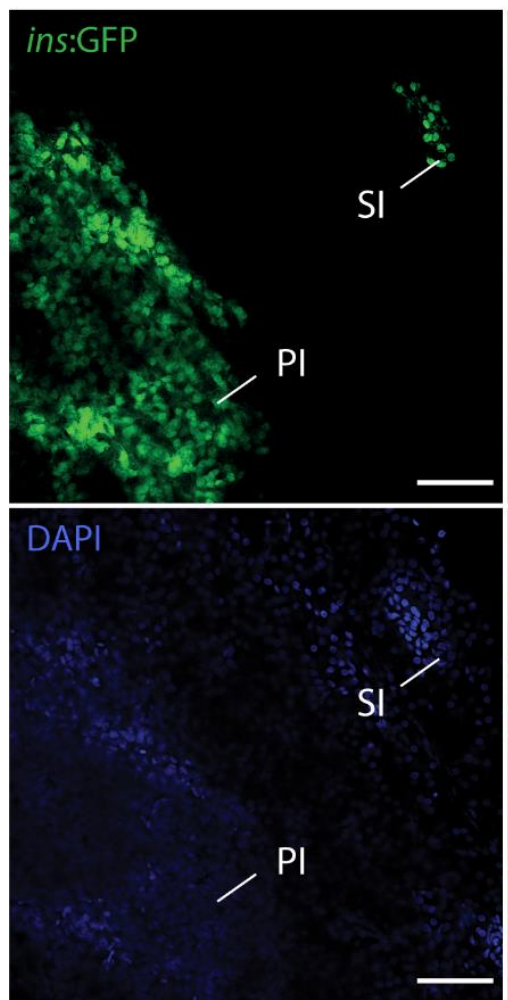

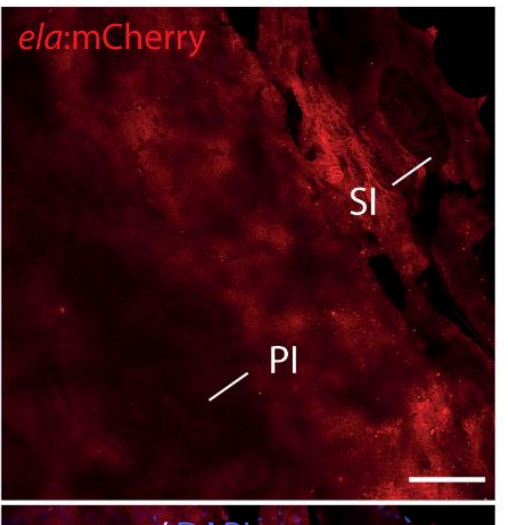

merge w/DAPI

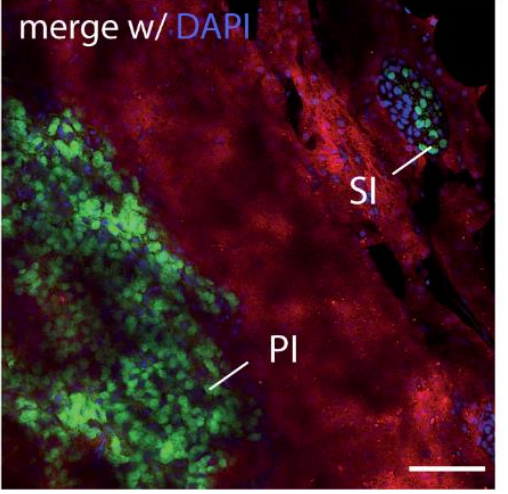

b

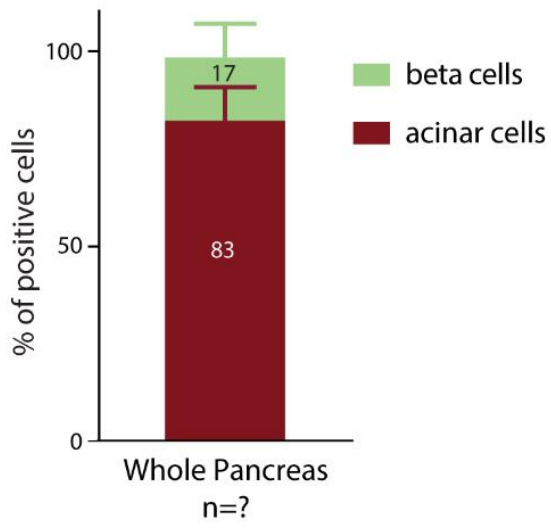

Supplementary figure 1. Basic constitution of the zebrafish adult pancreas. a) Confocal image of wholemounted pancreatic tissue from adult Tg(insulin:GFP, elastase:mCherry) zebrafish. The endocrine pancreas consists of a larger principal islet (PI) and smaller secondary islets (SI), where the insulin-producing beta-cells are visible (green), embedded within the pancreatic exocrine tissue, that itself is comprised of acinar cells (red) and a network of duct cells. Nuclei are stained with DAPI. Scale bar: $50 \mu \mathrm{m}$. b) Percentage of pancreatic endocrine beta-cells and exocrine acinar cells from $\mathrm{Tg}$ (insulin:GFP, elastase:mCherry), quantified by flow cytometry, from adult zebrafish pancreas $(n=5)$. 


\section{Supplementary figure 2}

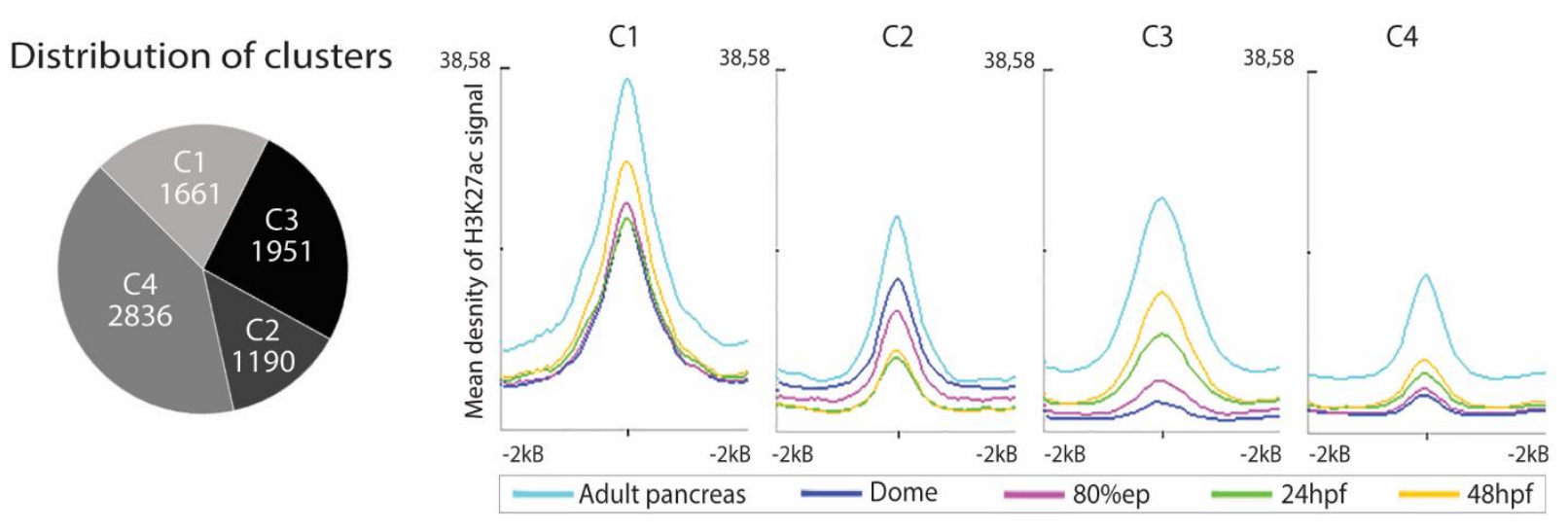

Supplementary figure 2. Left panel represents the number of sequences contained in each of the four clusters observed in DevE; C1: Cluster 1, C2: Cluster 2, C3: Cluster 3 and C4: Cluster 4. Right panel represents the mean density of $\mathrm{H} 3 \mathrm{~K} 27 \mathrm{Ac}$ signal for each cluster, centred in its summit and expanded $\pm 2 \mathrm{~kb}$, from adult pancreas and embryos at different developmental stages: dome, $80 \%$ epiboly, 24hpf and 48hpf. 


\section{Supplementary figure 3}

a

Association by proximity

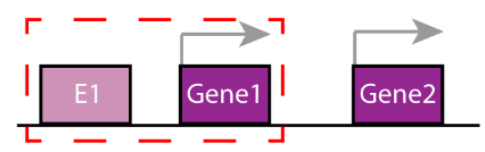

Pancreas specific enhancers (PsE)

\begin{tabular}{|l|c|c|}
\hline Tissue enrichment & Binomial FDR Q-value & Binomial fold enrichment \\
\hline Exocrine pancreas & $4,27 \mathrm{E}-09$ & 2,87 \\
\hline Blood & $1,10 \mathrm{E}-08$ & 2,05 \\
\hline Portion of organism & $1,10 \mathrm{E}-08$ & 2,05 \\
\hline
\end{tabular}

Pancreas enhancers (PsE + DevE)

\begin{tabular}{|l|c|c|}
\hline Tissue enrichment & Binomial FDR Q-value & Binomial fold enrichment \\
\hline Epidermis & $9,47 \mathrm{E}-36$ & 2,01 \\
\hline Blood vessel & $2,04 \mathrm{E}-34$ & 2,28 \\
\hline Epidermis & $1,17 \mathrm{E}-33$ & 2,01 \\
\hline
\end{tabular}

Association by chromatin interaction points

b
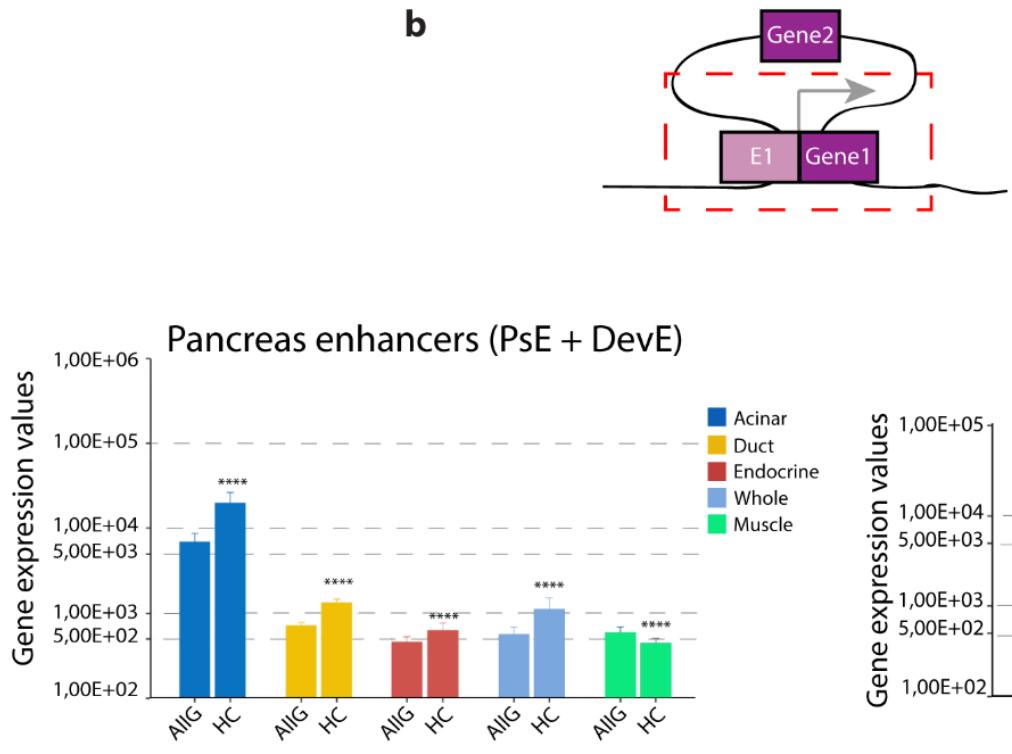

Developmental enhancers (DevE)
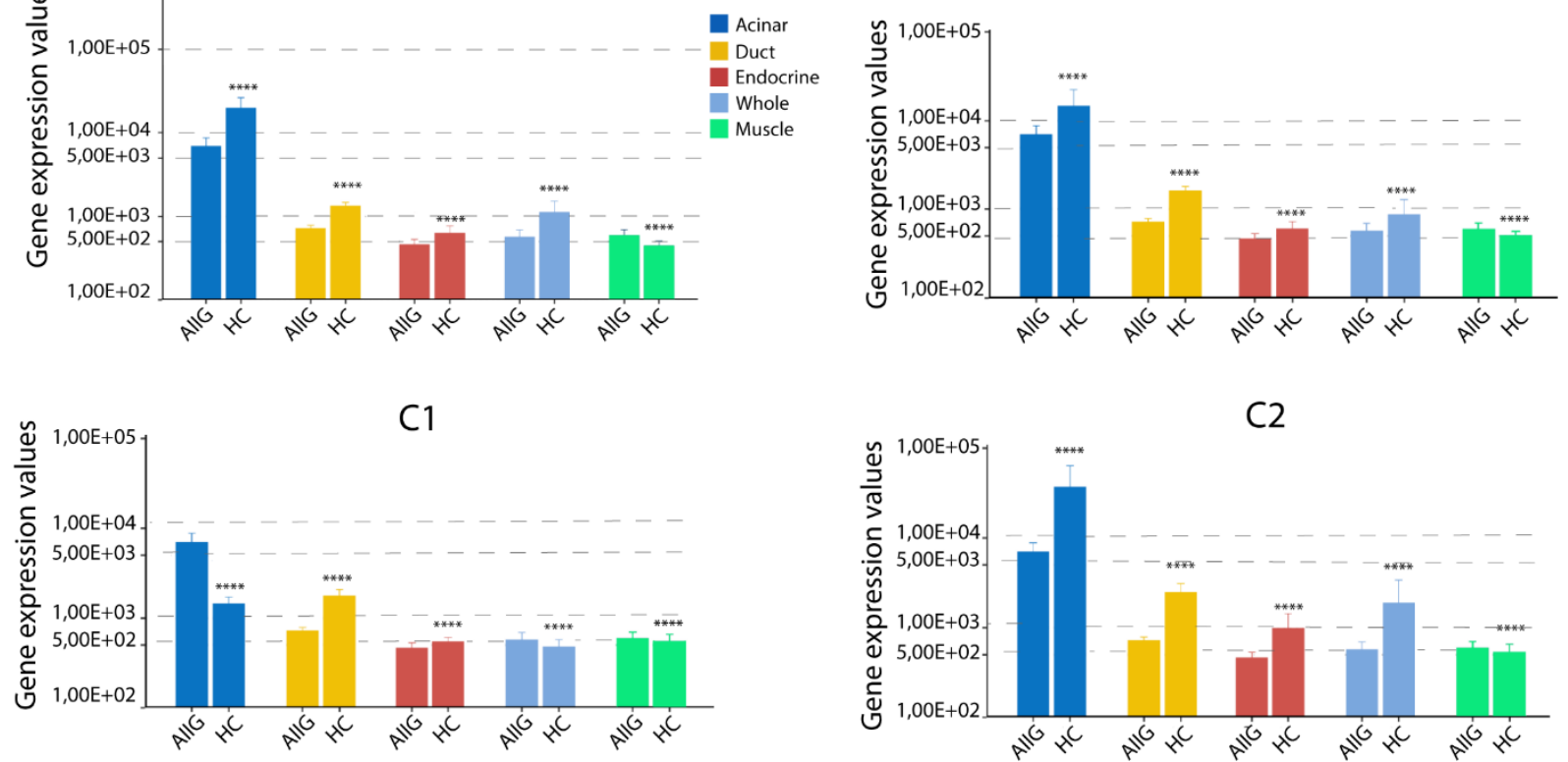

C3
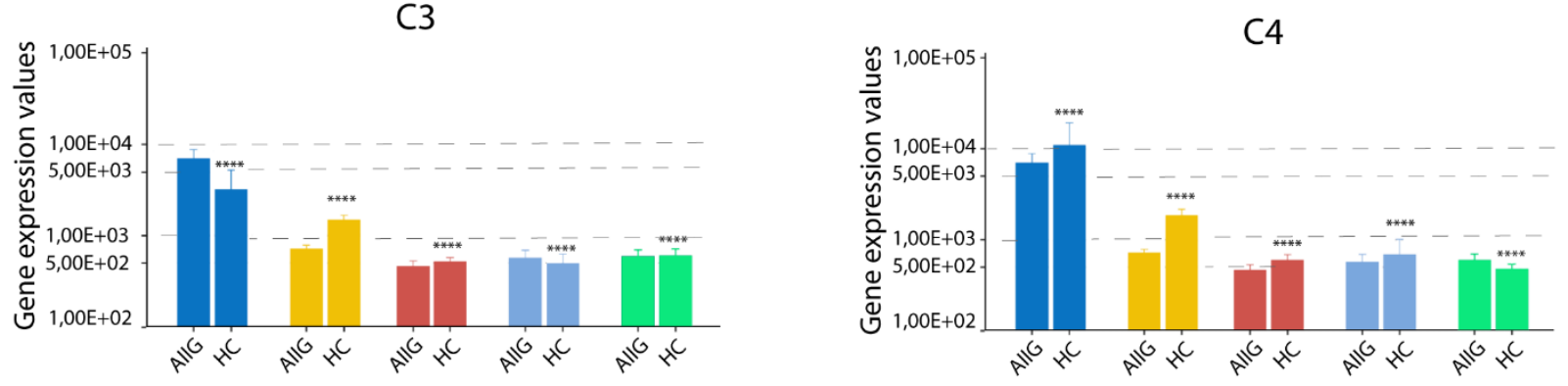
Supplementary figure 3. a) Upper panel: Schematic representation of gene-to-enhancer association by genomic proximity with GREAT ${ }^{1}$. Lower panel: Tissue specific expression enrichment for genes associated to PsE and PsE+DevE. b) Schematic representation of gene-to-enhancer association by chromatin interaction points defined by HiChIP for $\mathrm{H} 3 \mathrm{~K} 4 \mathrm{me} 3$ (HC). Average expression of genes interacting with different enhancer sets detected by $\mathrm{HC}$ in adult zebrafish pancreas, compared to the average expression of all genes (AllG), determined by RNA-seq from pancreatic cells (acinar, duct, endocrine), whole pancreas and muscle (control). Enhancer sets: pancreas specific enhancers (PsE), developmental enhancers (DevE), and clusters of developmental enhancers $\mathrm{C} 1, \mathrm{C} 2, \mathrm{C} 3$ and $\mathrm{C} 4$. Wilcoxon test, $p$-values $\leq 0.05$ considered significant. PsE+DevE: ****p<2e-16; DevE, C1-C4:**** $p<0,0001$. 


\section{Supplementary figure 4}
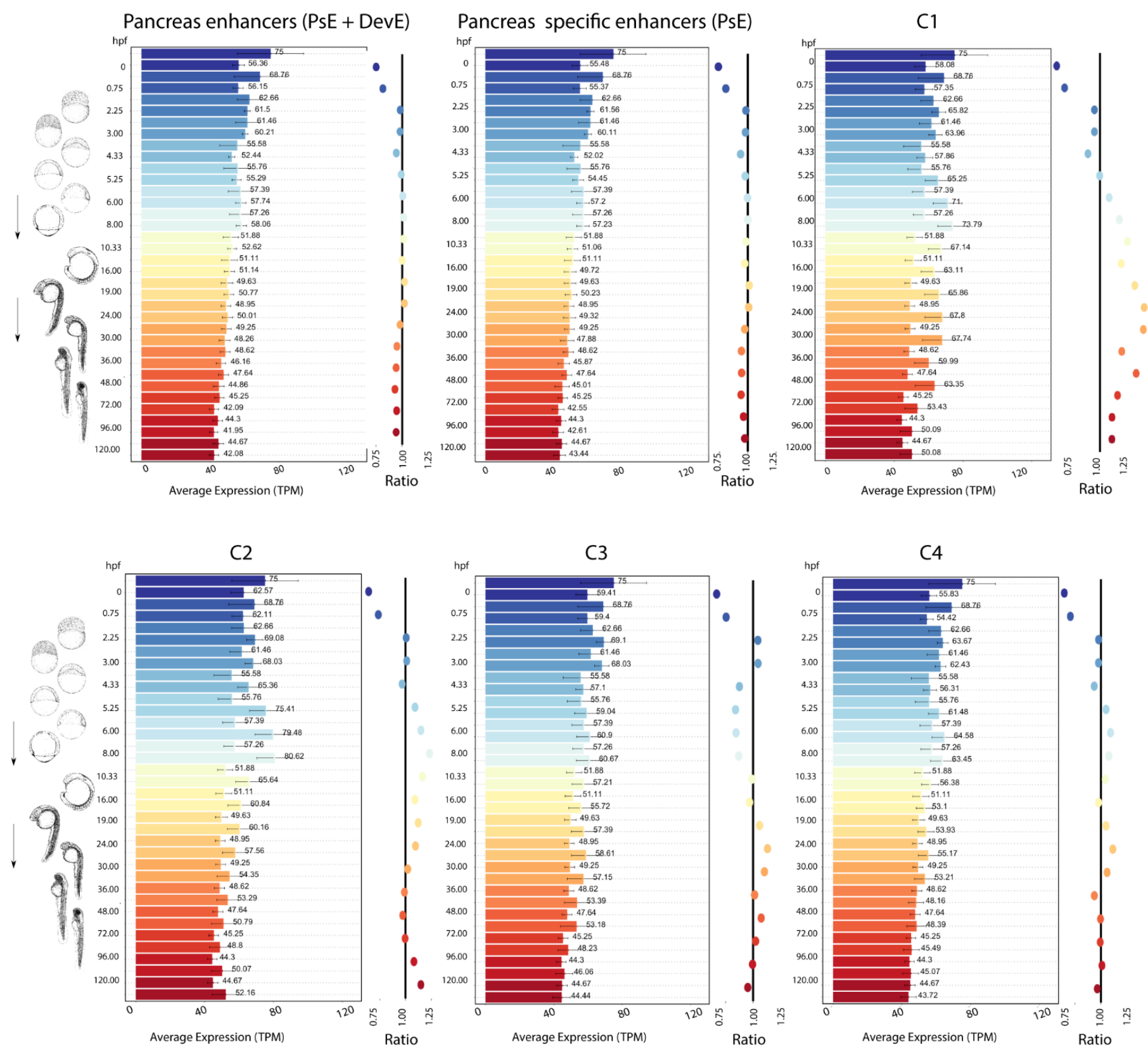

Supplementary figure 4. At the left side of each panel, the average gene expression (transcripts per million, TPM) detected from RNAseq from zebrafish embryos at different developmental stages ( 0 to $120 \mathrm{hpf})^{2}$ is plotted. The top bar of each colour is the average expression of genes associated to pancreas enhancers by HiChIP and bottom bar of each colour is the average expression of all genes as control, with the respective value depicted for each bar. On the right side of each panel is the depicted the ratio of the average expression of all genes as control by the average expression of genes associated to pancreas enhancers by HiChIP, maintaining the same colour code. 


\section{Supplementary figure 5}

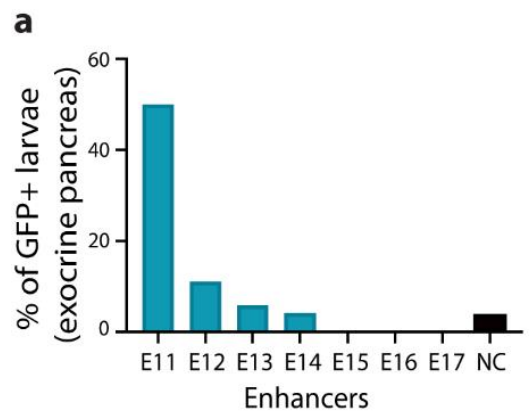

b
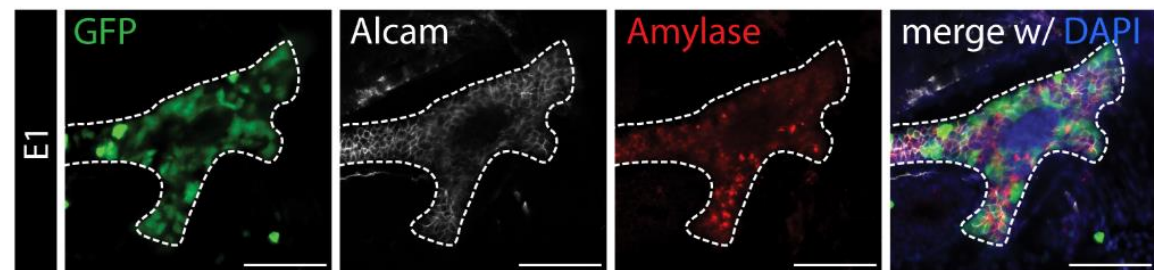

Enhancers

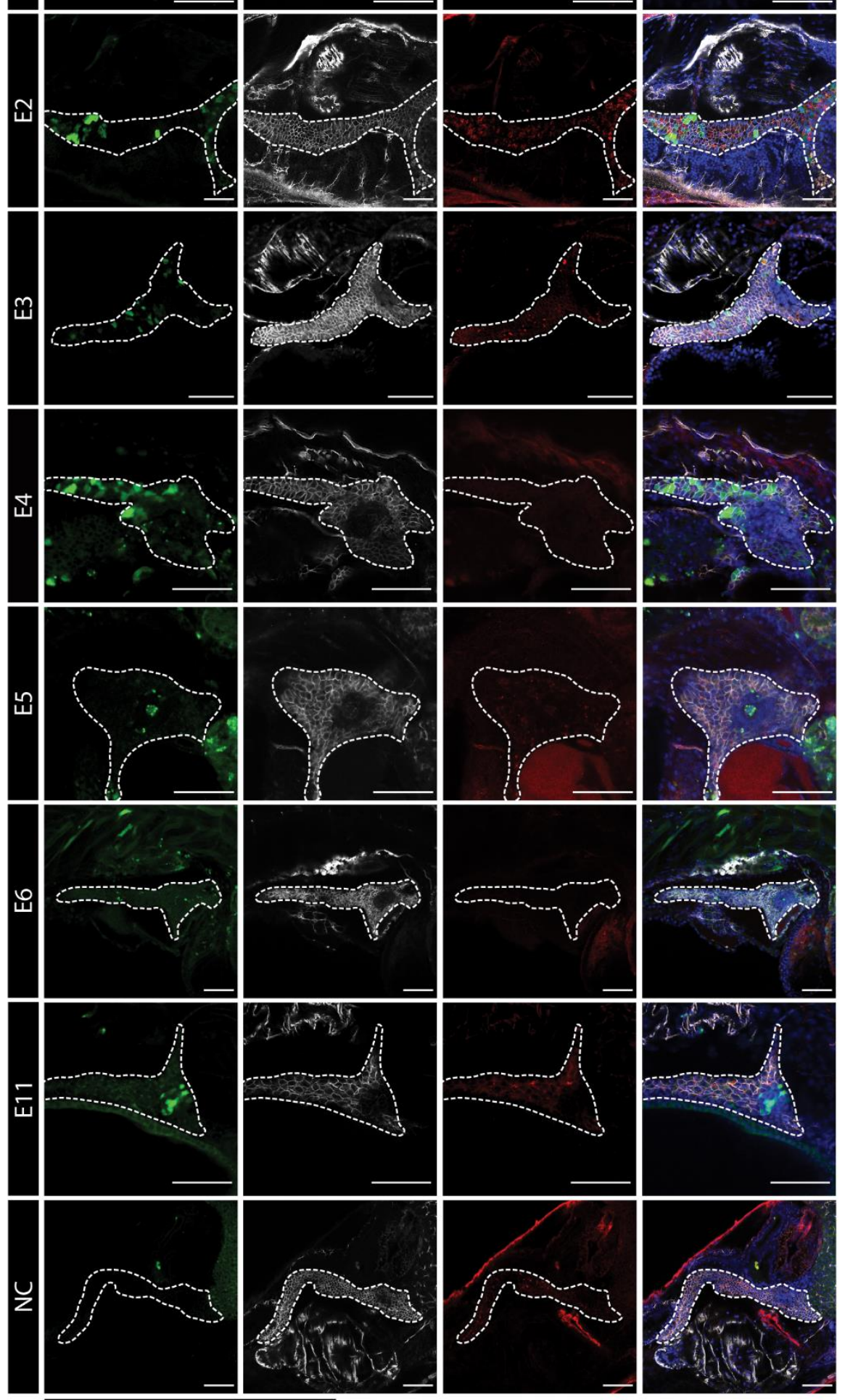

-----.- pancreatic domain 
Supplementary figure 5. In vivo validation of selected putative pancreatic enhancers, identified by ATAC and ChIP-seq data, by transgenesis assays in zebrafish embryos injected with Z48 vector carrying putative enhancer sequences and the empty vector was used as negative control (NC). a) Percentage of embryos with GFP expression in zebrafish pancreas for sequences (E11 to 17) with low H3K27ac ChIP signal value: ((-log10(pvalue)<35). Chi-square test, $\mathrm{p}$-values $<0.05$ were considered significant. $\mathrm{NC}$ is the negative control (empty vector). b) Representative confocal images of transgenic F0 larvae for all validated enhancers (E1-E6 and E11); whole mount immunohistochemistry of 10-12 dpf zebrafish larvae showing enhancer activity, in the form of GFP expression (green), within the pancreatic domain (dashed white line), in comparison to the NC. The exocrine pancreas is stained with anti-Alcam (white) and anti-Amylase (red) antibodies. Nuclei are stained with DAPI. Scale bar, $60 \mu \mathrm{m}$. 


\section{Supplementary figure 6}

a

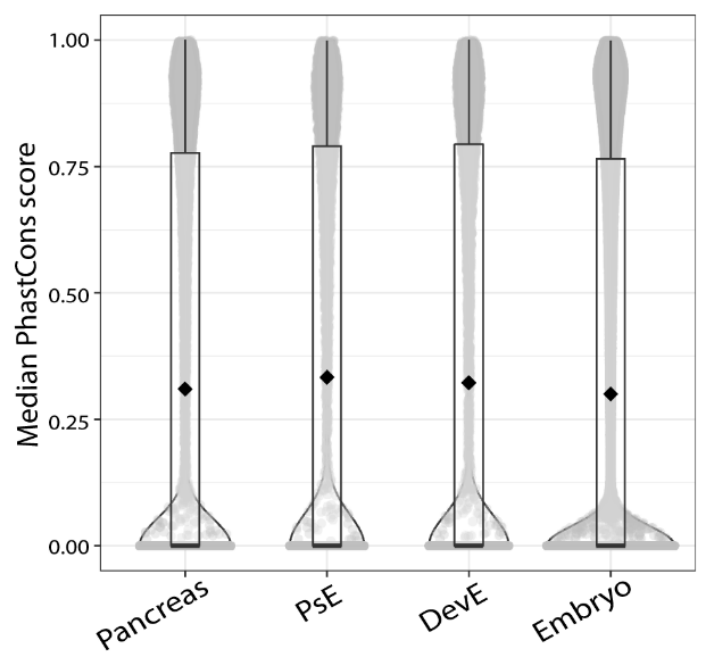

b

Zebrafish and human genes associated to super-enhancers

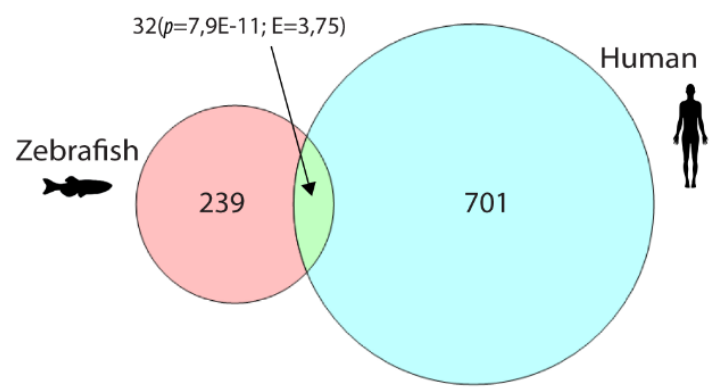

C

Gene ontology for zebrafish genes associated to super-enhancers

\begin{tabular}{|c|c|c|c|c|c|c|}
\hline \multirow[b]{2}{*}{ Molecular Function } & \multirow{2}{*}{$\begin{array}{c}\text { Danio rerio (REF) } \\
\#\end{array}$} & \multicolumn{5}{|c|}{ Zebrafish genes associated to super-enhancers } \\
\hline & & \# & expected & Fold Enrichment & $+1-$ & P value \\
\hline transcription factor binding & 203 & 10 & 2,14 & \begin{tabular}{|l|l}
4,68 \\
\end{tabular} & + & $3,88 \mathrm{E}-02$ \\
\hline binding & 5971 & 93 & 62,81 & 1,48 & + & $1,50 \mathrm{E}-02$ \\
\hline transcription coregulator activity & 229 & 11 & 2,41 & 4,57 & + & $2,14 \mathrm{E}-02$ \\
\hline transcription regulator activity & 1167 & 41 & 12,27 & 3,34 & + & $1,12 \mathrm{E}-08$ \\
\hline RNA polymerase II proximal promoter sequence-specific DNA binding & 243 & 11 & 2,56 & 4,30 & + & $3,57 \mathrm{E}-02$ \\
\hline transcription regulatory region DNA binding & 390 & 16 & 4,10 & 3,90 & + & $2,88 \mathrm{E}-03$ \\
\hline DNA binding & 837 & 23 & 8,80 & 2,61 & + & $1,72 \mathrm{E}-02$ \\
\hline nucleic acid binding & 1865 & 47 & 19,62 & 2,40 & + & $1,38 \mathrm{E}-05$ \\
\hline organic cyclic compound binding & 1959 & 47 & 20,61 & 2,28 & + & $5,99 \mathrm{E}-05$ \\
\hline regulatory region nucleic acid binding & 391 & 16 & 4,11 & 3,89 & + & $2,97 \mathrm{E}-03$ \\
\hline RNA polymerase II regulatory region sequence-specific DNA binding & 334 & 13 & 3,51 & 3,70 & + & $3,57 \mathrm{E}-02$ \\
\hline RNA polymerase II regulatory region DNA binding & 336 & 14 & 3,53 & 3,96 & + & $9,30 \mathrm{E}-03$ \\
\hline RNA polymerase II transcription factor activity, sequence-specific DNA binding & 461 & 16 & 4,85 & 3,30 & + & $2,06 \mathrm{E}-02$ \\
\hline DNA-binding transcription factor activity & 1067 & 35 & 11,22 & 3,12 & + & $2,12 \mathrm{E}-06$ \\
\hline Unclassified & 13833 & 111 & 145,50 & 0,76 & - & $0,00 \mathrm{E} 00$ \\
\hline
\end{tabular}

11

Gene ontology for human genes associated to super-enhancers

\begin{tabular}{|c|c|c|c|c|c|c|}
\hline \multirow[b]{2}{*}{ Molecular Function } & \multirow{2}{*}{\begin{tabular}{|c|} 
Homo sapiens (REF) \\
$\#$
\end{tabular}} & \multicolumn{5}{|c|}{ Human genes associated to super-enhancers } \\
\hline & & \# & expected & Fold Enrichment & $+1-$ & P value \\
\hline transcriptional repressor activity, RNA polymerase II proximal promoter sequence-specific DNA & 68 & 15 & 4,18 & 3,59 & + & $3,48 \mathrm{E}-02$ \\
\hline RNA polymerase II transcription factor activity, sequence-specific DNA binding & 365 & 58 & 22,44 & 2,58 & + & $5,35 \mathrm{E}-07$ \\
\hline DNA-binding transcription factor activity & 983 & 105 & 60,44 & 1,74 & + & $1,11 \mathrm{E}-04$ \\
\hline transcription regulator activity & 1100 & 115 & 67,64 & 1,70 & + & $8,32 \mathrm{E}-05$ \\
\hline RNA polymerase II proximal promoter sequence-specific DNA binding & 193 & 32 & 11,87 & 2,70 & + & $1,74 \mathrm{E}-03$ \\
\hline transcription regulatory region DNA binding & 330 & 46 & 20,29 & 2,27 & + & $8,14 \mathrm{E}-04$ \\
\hline regulatory region nucleic acid binding & 331 & 46 & 20,35 & 2,26 & + & $8,54 \mathrm{E}-04$ \\
\hline RNA polymerase II regulatory region sequence-specific DNA binding & 279 & 40 & 17,16 & 2,33 & + & $2,16 \mathrm{E}-03$ \\
\hline RNA polymerase II regulatory region DNA binding & 282 & 41 & 17,34 & 2,36 & + & $1,32 \mathrm{E}-03$ \\
\hline Unclassified & 11259 & 606 & 692,29 & 0,88 & - & $0,00 \mathrm{E} 00$ \\
\hline G-protein coupled receptor activity & 563 & 9 & 34,62 & 0,26 & - & $3,15 \mathrm{E}-04$ \\
\hline transmembrane signaling receptor activity & 826 & 20 & 50,79 & 0,39 & - & $8,59 \mathrm{E}-04$ \\
\hline
\end{tabular}

d
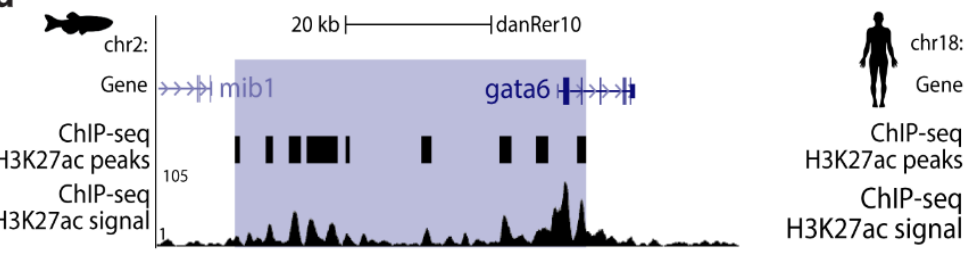

$100 \mathrm{~kb}$

hg19

GATA6 HHH $\rightarrow \rightarrow \rightarrow-1$

| ||

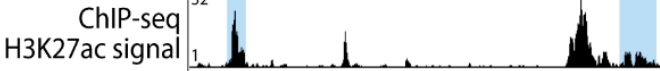


Supplementary figure 6. a) Distribution of the median PhastCons scores for each zebrafish putative enhancer sequence active in adult pancreas (14301), adult pancreas only (PsE, 6918), adult pancreas and embryo (DevE, 8368) and in embryo (Embryo, 65871). Putative active enhancer sequences converted from DanRer10 to DanRer7 (liftOver) to match the available conservation scores for zebrafish and 7 vertebrates. The median value is zero for the 4 enhancer sets (lower bar of the boxplots) since most sequences are not conserved, while the average is, respectively, 0.31, 0.33, 0.32, 0.30 (back diamond inside the boxplot). b) Zebrafish and human genes associated to super-enhancers, identified by $\operatorname{ROSE}^{3} \mathbf{c}$ ) Gene ontology for genes associated to super-enhancers in zebrafish (upper table) and human (lower table). d) H3k27ac profile of the landscape of a gene important in pancreatic development in zebrafish (gata6; left) and human (GATA6; right); super-enhancers are highlighted in purple (zebrafish) and blue (human). 


\section{Supplementary figure 7}

a

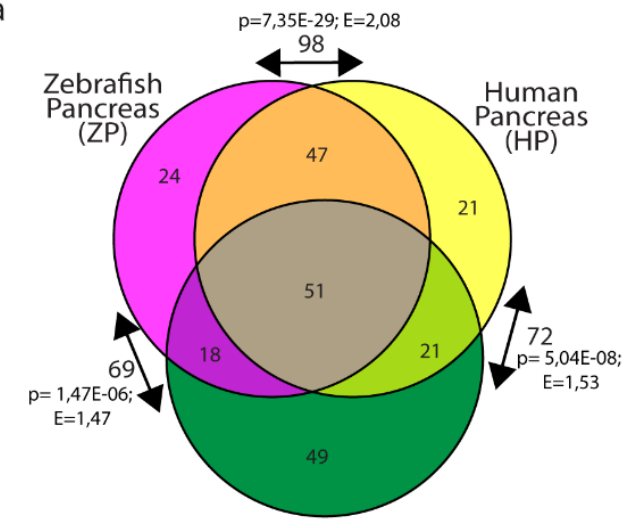

$24 \mathrm{HPF}$

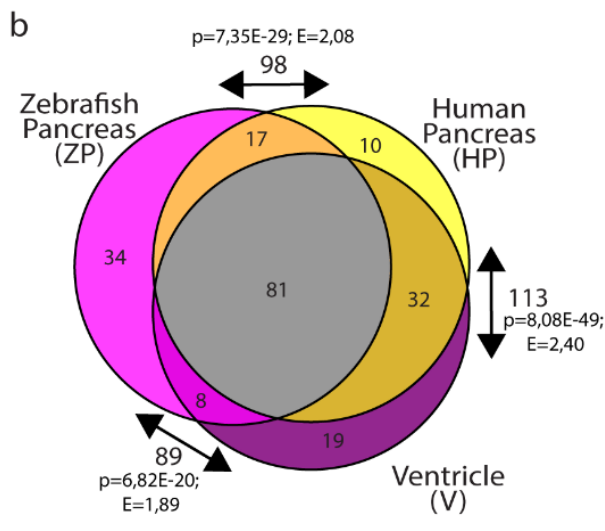

e $Z P, V$

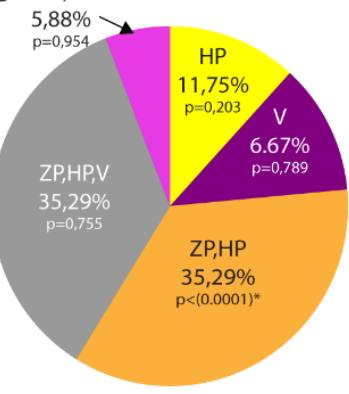

Pancreas TFs

$\begin{array}{ll}\text { Ascl1 } & \text { Nkx6.1 } \\ \text { Foxa1 } & \text { MafA } \\ \text { FoxA2 } & \text { NeuroD1 } \\ \text { Gata4 } & \text { Pdx1 } \\ \text { Gata6 } & \text { Rfx3 } \\ \text { Hfn1b } & \text { Rpbj } \\ \text { Hnf4 } & \text { Sox9 }\end{array}$

(V)

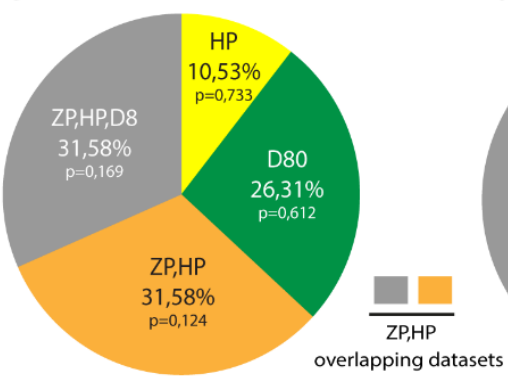

Pancreas TFs

$\begin{array}{ll}\text { Ascl1 } & \text { Isl1 } \\ \text { FoxA1 } & \text { MafA } \\ \text { FoxA2 } & \text { MafB } \\ \text { Gata4 } & \text { NeuroD1 } \\ \text { Gata6 } & \text { Nkx6.1 } \\ \text { Hfn1b } & \text { Pdx1 } \\ \text { Hnf4 } & \text { Rpbj } \\ \text { Hnf6 } & \text { Sox9 }\end{array}$

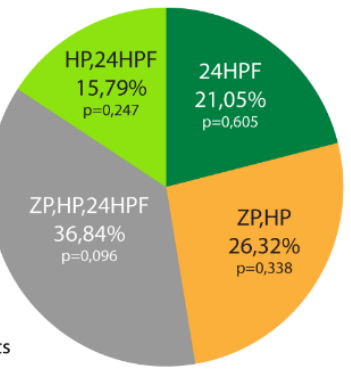

Pancreas TFs

\begin{tabular}{ll} 
Ascl1 & Nkx6.1 \\
Foxa1 & MafA \\
Foxa2 & NeuroD1 \\
Gata4 & Pdx1 \\
Gata6 & Ptf1A \\
Hfn1b & Rfx3 \\
Hnf4 & Rpbj \\
Isl1 & Sox9 \\
\hline
\end{tabular}

\begin{tabular}{|c|c|c|c|c|c|c|c|c|}
\hline \multicolumn{3}{|c|}{ PsE } & \multicolumn{3}{|l|}{ C1 } & \multicolumn{3}{|l|}{ C2 } \\
\hline Motif & Name & P-value & Motif & Name & P-value & Motif & Name & P-value \\
\hline CCTTATCTE두술 & GATA6 & $1 \mathrm{E}-96$ & 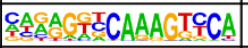 & HNF4a & $1 E-60$ & ATACICCCACCT & CTCF & $1 \mathrm{E}-24$ \\
\hline AGAIAACA & GATA3 & $1 \mathrm{E}-94$ & CIAGGTCAAAGGTCA & PPARa & $1 \mathrm{E}-51$ & 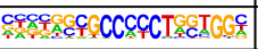 & BORIS & $1 \mathrm{E}-19$ \\
\hline CAGATAAGEA & GATA1 & $1 \mathrm{E}-91$ & 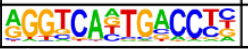 & FXR & $1 \mathrm{E}-48$ & 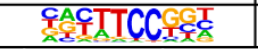 & FLI & $1 \mathrm{E}-13$ \\
\hline
\end{tabular}

\begin{tabular}{|c|c|c|c|c|c|}
\hline \multicolumn{3}{|c|}{ C3 } & \multicolumn{3}{|c|}{ C4 } \\
\hline Motif & Name & P-value & Motif & Name & P-value \\
\hline CAGATAAGGA & GATA1 & $1 E-32$ & CAGATAAGGA & GATA1 & $1 \mathrm{E}-29$ \\
\hline CCTIATCT두숫 & GATA6 & $1 \mathrm{E}-31$ & AACCGGAAGI & ETV1 & $1 \mathrm{E}-21$ \\
\hline CCAGATAAGA & GATA4 & $1 E-31$ & TCCTTATCTC & GATA2 & $1 E-21$ \\
\hline
\end{tabular}


Supplementary figure 7. a-b) Regions enriched in ATAC-seq and H3k27ac signal from zebrafish pancreas (ZP), human pancreas (HP), 24hpf zebrafish embryos (24HPF) and human ventricle (V) were investigated for TFBS motifs (SupplementaryTable14 and 15). The top 140 enriched TFBS motifs from each tissue were selected and the overlap of those sets was analysed. Arrows: number of TFBS motifs shared between two different groups, the enrichment of TFBS motifs and respective $p$-value for each intersection. Number of TFBS motifs identified in each group/intersection. a) ZP, HP and 24HPF; b) ZP, HP, V. c-e) The motifs corresponding to 25 pancreas transcription factors (Pancreas TFs) selected from literature were analysed for their representation in $\mathrm{H} 3 \mathrm{~K} 27 \mathrm{ac}$ peaks filtered with ATAC-seq peaks among the different zebrafish (ZP, 24HPF, D80) and human tissues (HP, V), and tissue-intersections shown in a-b). The distribution of these TFBS motifs through the different tissues is shown in the graph with the percentage and the respective $p$-value indicated for each group (top). The TFs present in the tissues, from each graph, are depicted in the table (bottom). c) ZP, HP and dome+80\%epiboly (D80); d) ZP, HP and 24hpf; e) ZP, HP and V. Statistical significance was assessed by Chi-square test, $p$-values $<0.05$ were considered significant. f) List of top three TFBS motifs enriched in H3K27ac ChIP-seq data, for the different enhancer sets: pancreas specific enhancers (PsE) and clusters of developmental enhancers $\mathrm{C} 1, \mathrm{C} 2, \mathrm{C} 3$ and $\mathrm{C} 4$, with the respective $p$-value. 


\section{Supplementary figure 8}

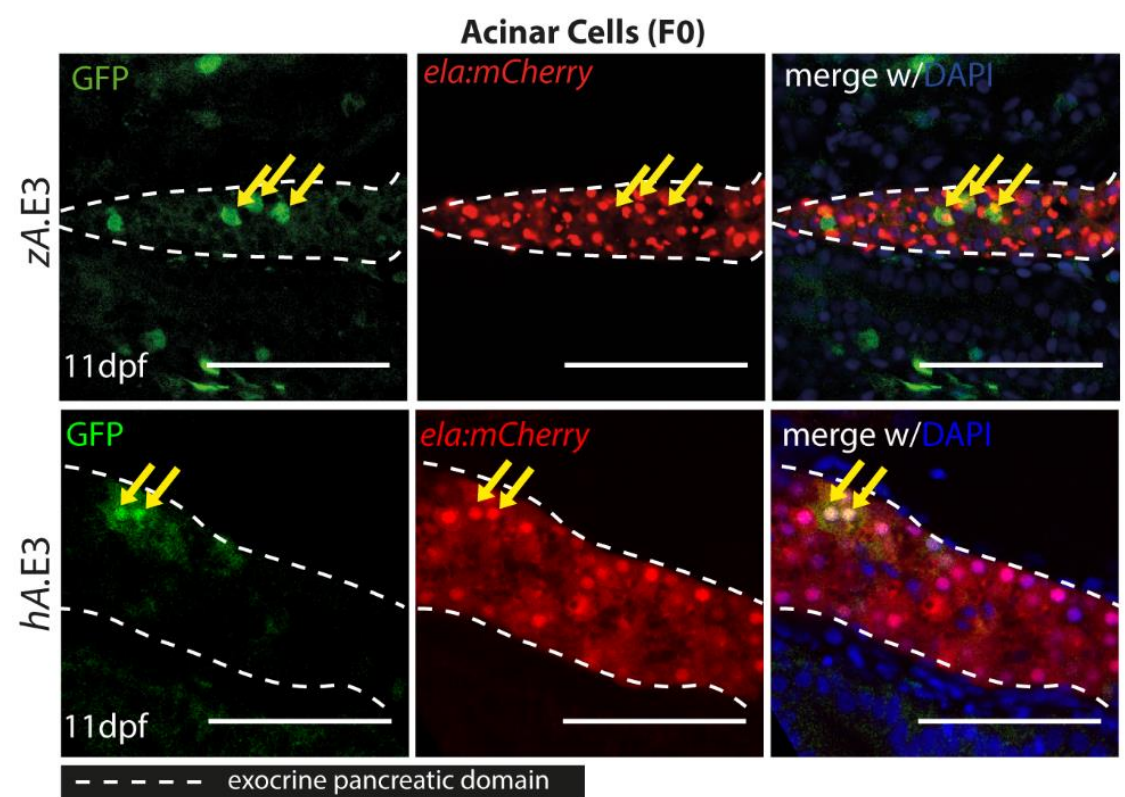

Duct Cells (F0)

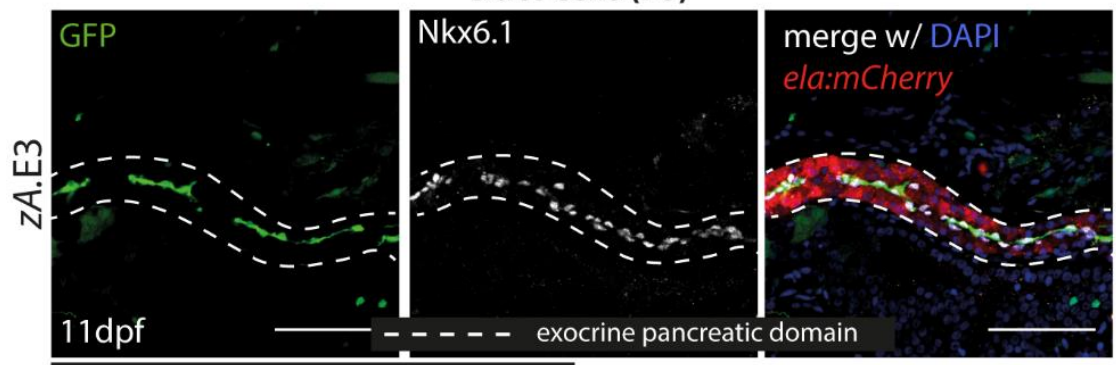

- - - - - exocrine pancreatic domain

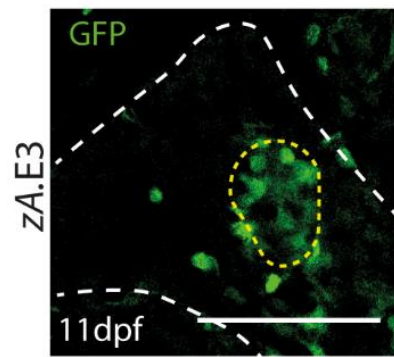

\section{Endocrine Cells}
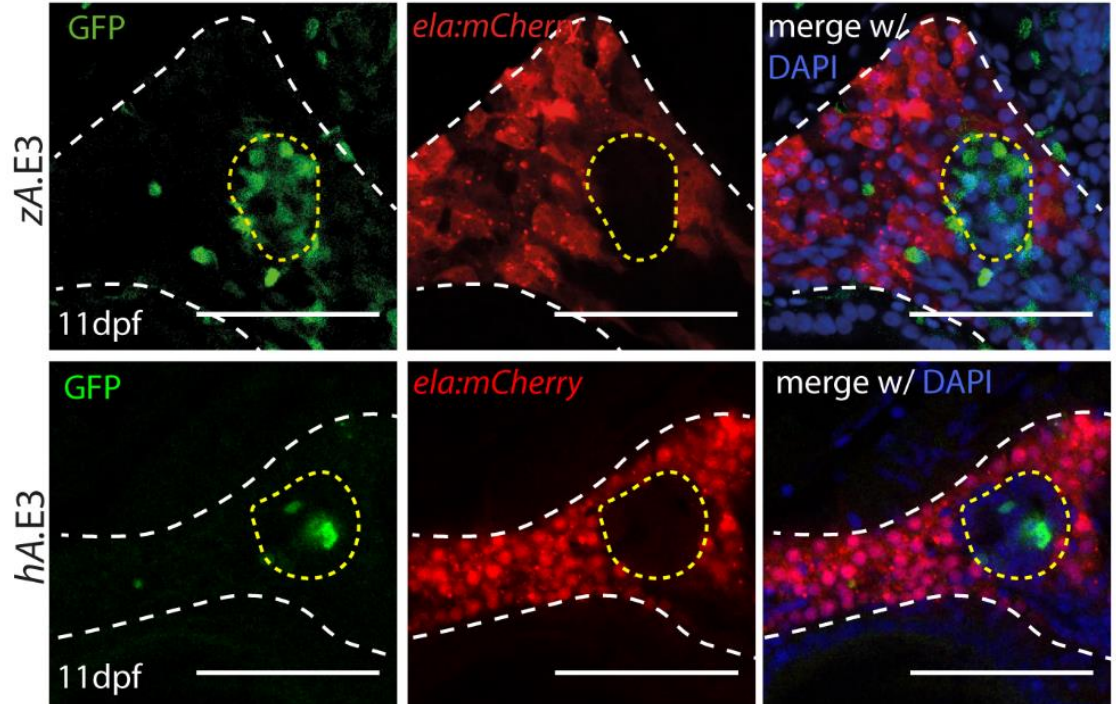

- - - - - exocrine pancreatic domain

.......... endocrine pancreatic domain 
Supplementary figure 8. Human and zebrafish ARIDIA/aridlab enhancers drive expression in various pancreatic cell types. Confocal images of zebrafish pancreas (white dashed line), at 11dpf, from F0 $\operatorname{Tg}$ (ela:mCherry) embryos injected with Z48 vector carrying zebrafish zAridE3 (zA.E3) or human hAridE3 (hA.E3) enhancers. The top panel shows elastase-producing acinar cells (red), the middle panel shows NKX6.1positive duct cells (white), and the bottom panel shows the endocrine pancreas domain (yellow dashed line). In all panels, the exocrine pancreas domain is indicated with a white dashed line and nuclei are stained with DAPI (blue). Scale bar: $60 \mu \mathrm{m}$. 


\section{Supplementary figure 9}
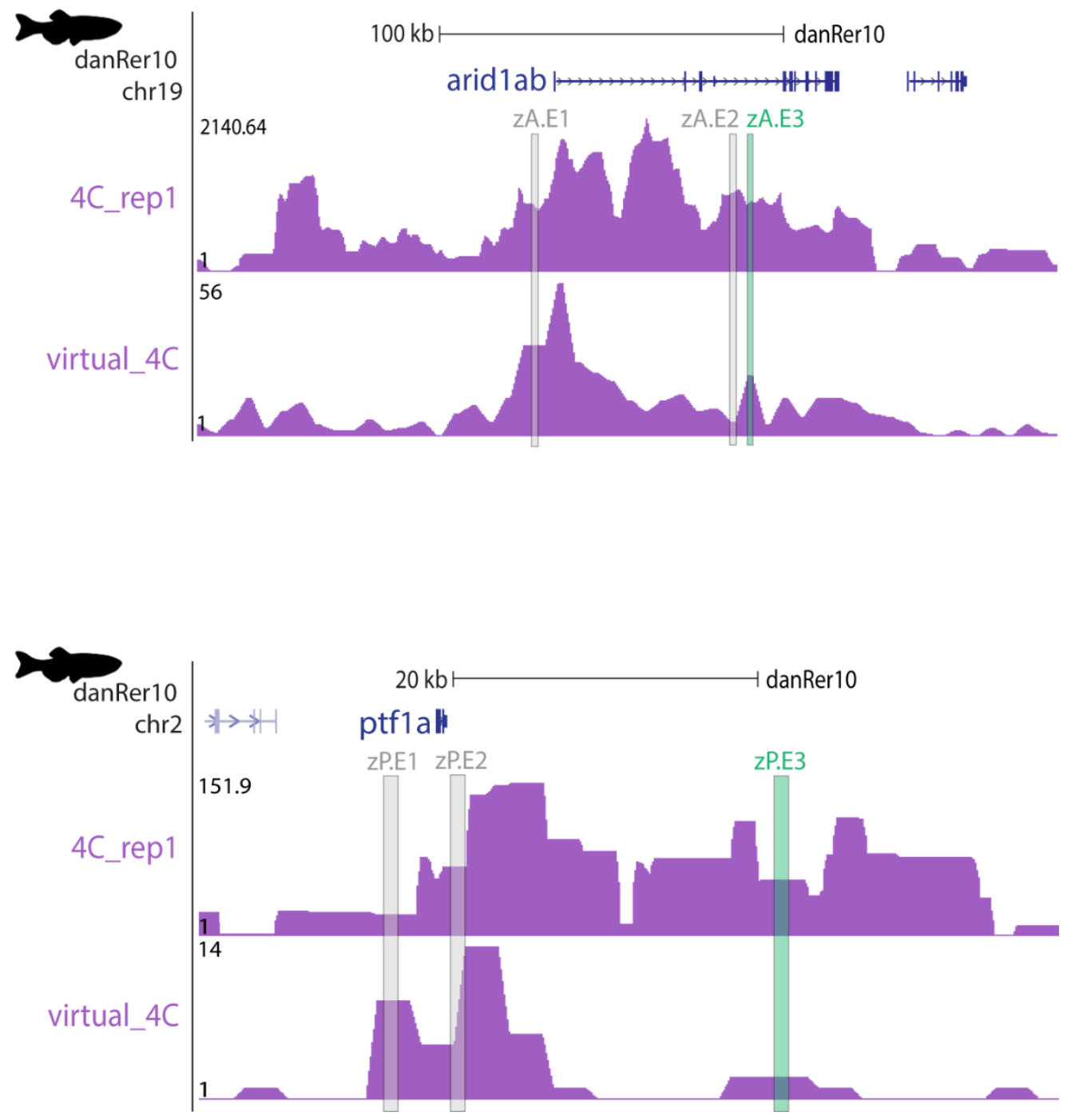

Supplementary figure 9. Genomic landscape of zebrafish aridla $a$ gene (top) and ptfla gene (bottom), showing aridlab 4C interactions (purple) from 4C-seq replicates and virtual 4C from HiChIP for H3K4me3 in adult zebrafish pancreas. Tested putative enhancers are highlighted in grey, zA.E1 (zAridE1) and zA.E2 (zAridE2) and green zA.E3 (zAridE3). 
bioRxiv preprint doi: https://doi.org/10.1101/2020.04.27.064220; this version posted April 28, 2020. The copyright holder for this preprint (which was not certified by peer review) is the author/funder. All rights reserved. No reuse allowed without permission.

\section{Supplementary figure 10}

a

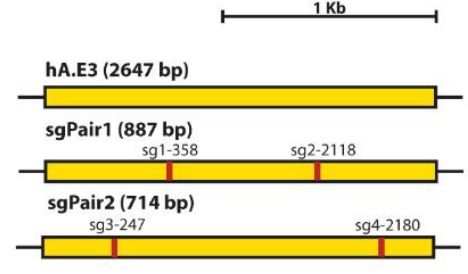

b

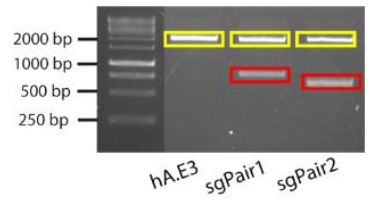

C

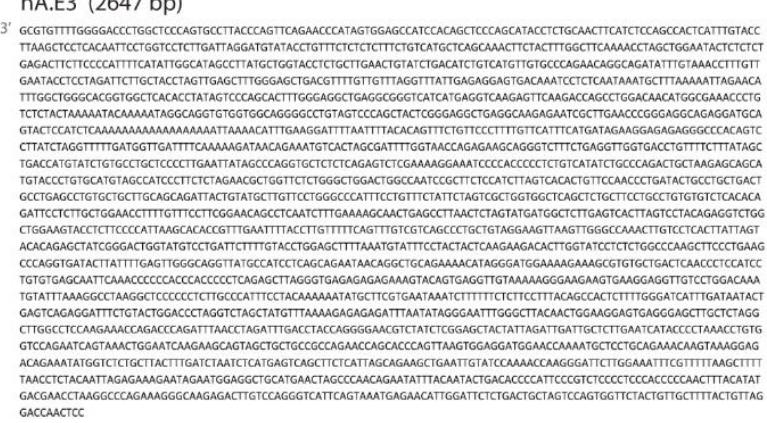

sgPair2 (714bp)

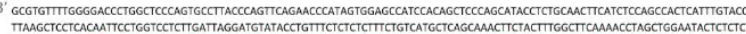

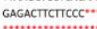

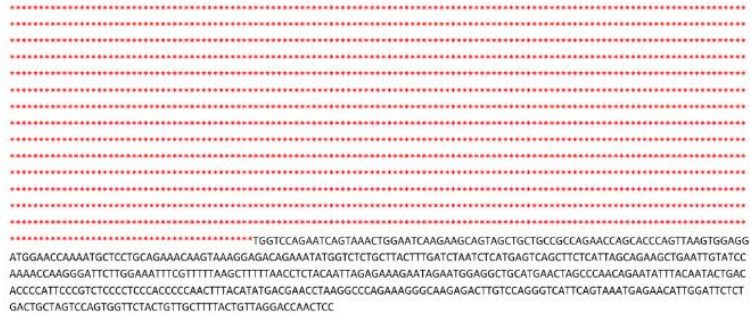

d

hA.E3
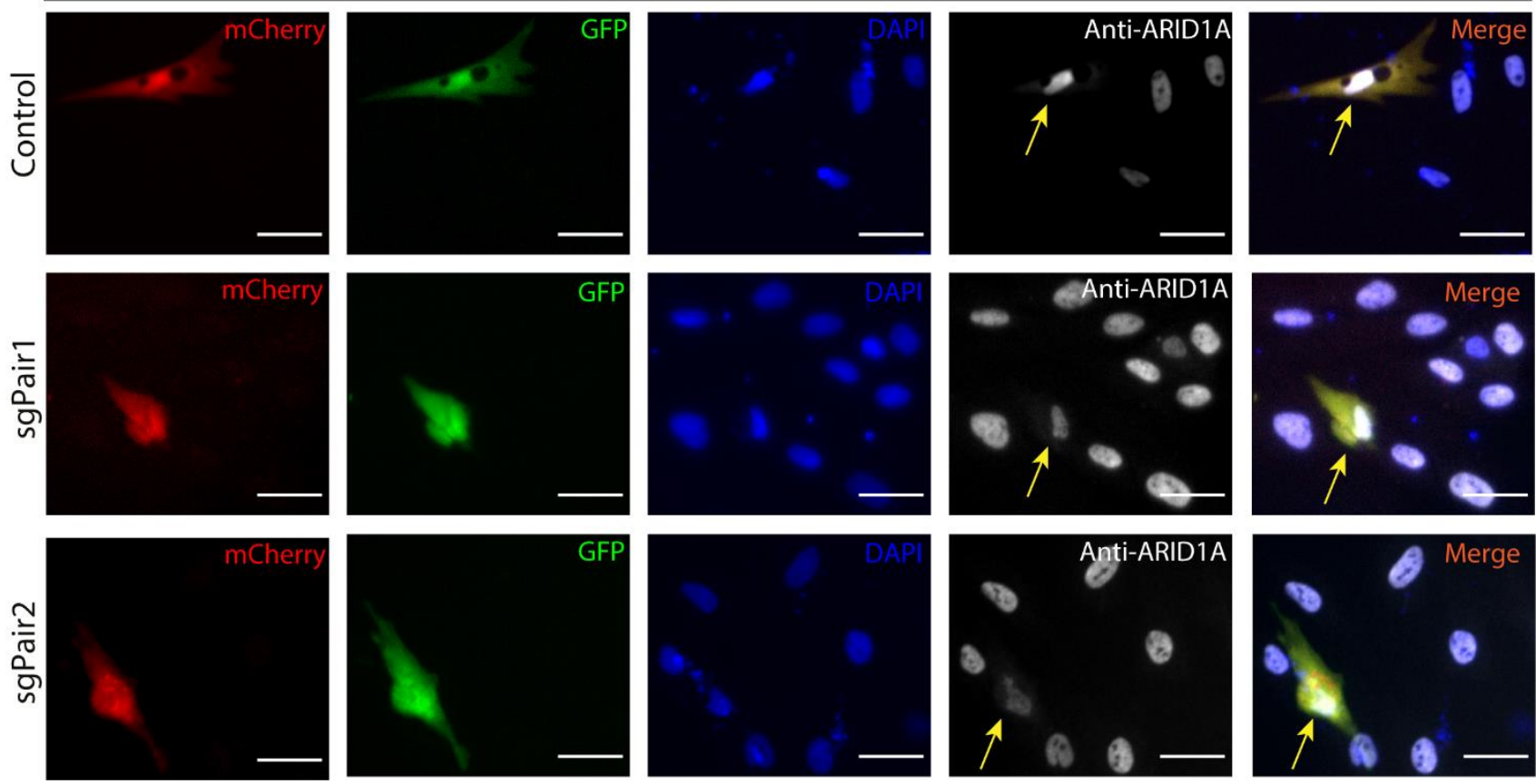
Supplementary figure 10. a) Schematic depiction of the targeting strategy for deletion of the hA.E3 (hAridE3) locus, indicating the CRISPR sgRNA target sites (red). b) Agarose gel showing the wild-type (yellow) and deleted (red) PCR amplified hA.E3 sequence after gene editing with the respective sgRNA pair. c) DNA sequencing results of the indicated genomic PCR products (yellow and red boxes). d) Representative fluorescent microscopy images of transfected hTERT-HPNE human cells, defined by the co-expression of GFP and mCherry (yellow arrows). Nuclei were stained with DAPI (blue) and anti-ARID1A antibody (white). The yellow arrows are pointing to the double transfected cell. Images were captured with Leica DMI6000 FFW microscope. Scale bar: $40 \mu \mathrm{m}$. 


\section{Supplementary figure 11}

a
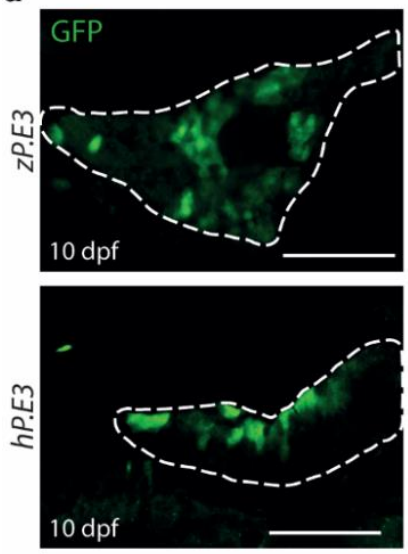

- - - - pancreatic domain b
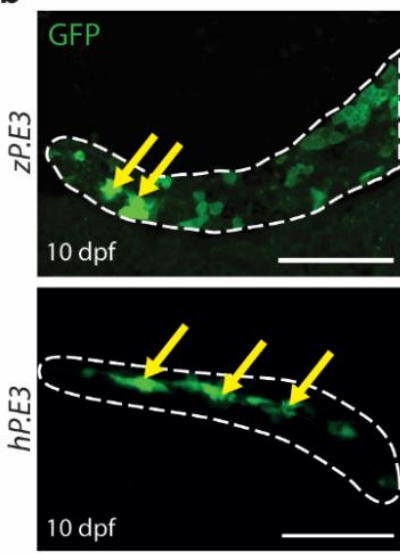

- - - - - - pancreatic domain
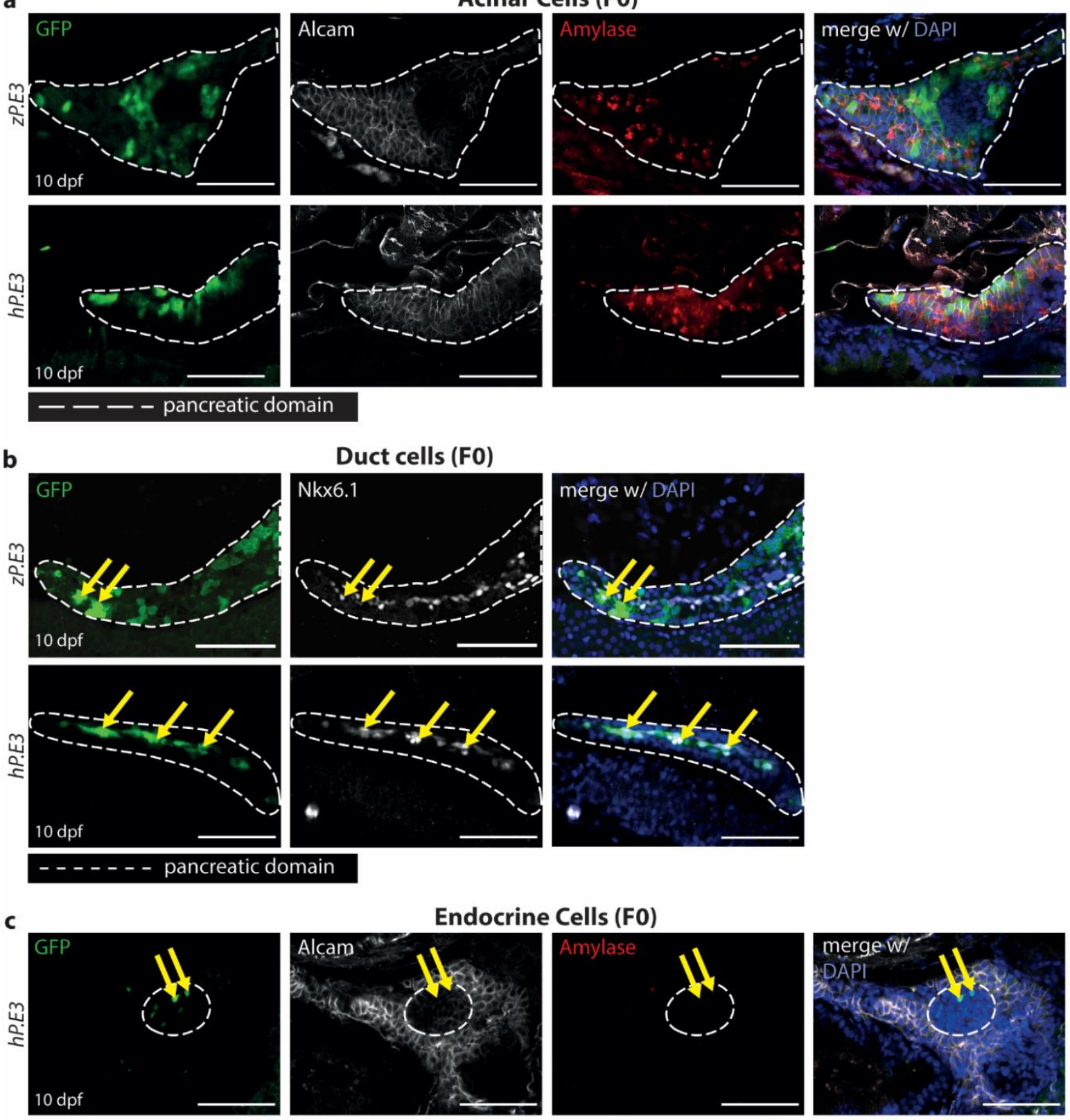

Duct cells (F0)
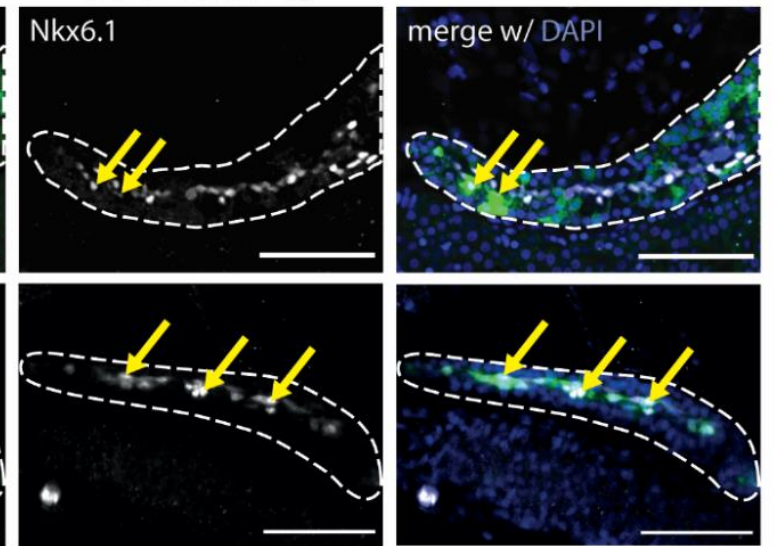

--------- endocrine pancreas domain

d
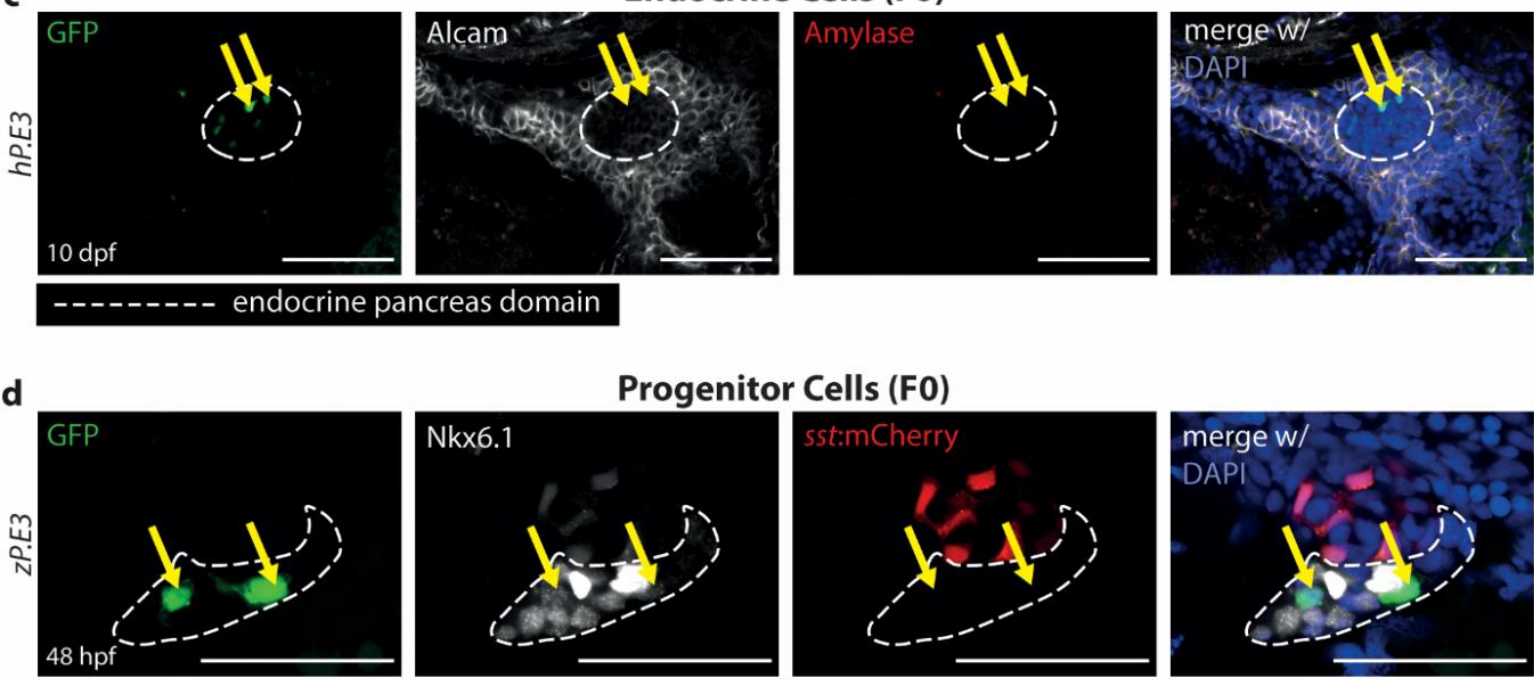

\section{Progenitor Cells (F0)}
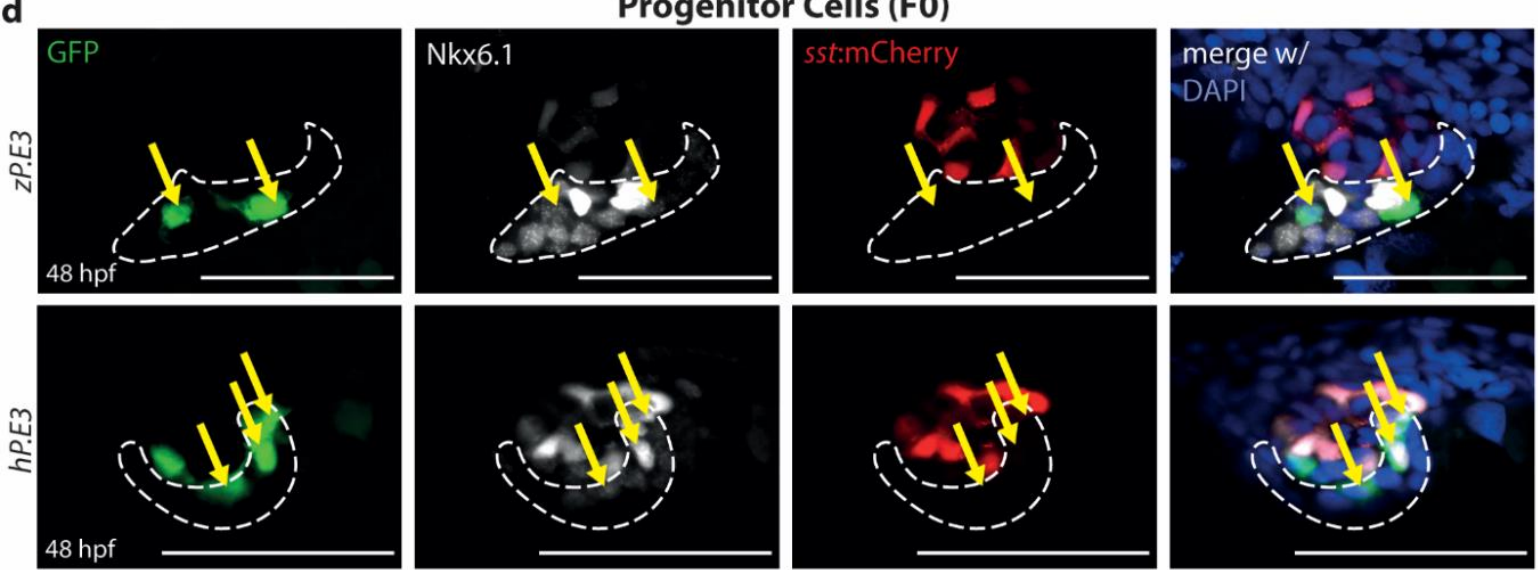
Supplementary figure 11. Human and zebrafish distal PTF la/ptfla enhancers drive similar reporter expression in various pancreatic cell types. a-d) Representative confocal images of GFP expression (green) driven by zebrafish zP.E3(zptf1aE3) or human hP.E3 (hptf1aE3) sequences in F0 10dpf zebrafish larvae in a) pancreatic acinar cells, stained with anti-Alcam (white) and anti-Amylase (red), b) duct cells, stained with anti-NKX6.1 (white) and c) endocrine cells, delimited by absence of staining of acinar cell markers. d) Pancreatic progenitor cells of 48hpf F0 zebrafish embryos stained with anti-NKX6.1 (white), adjacent to the endocrine principal islet identified by somatostatin expression in differentiated delta-cells, using $\mathrm{Tg}$ (somatostatin:mCherry) zebrafish. Nuclei are stained with DAPI (blue). Yellow arrows point to localization of GFP within the respective identified tissues (white dashed line). Scale bar $60 \mu \mathrm{m}$. 


\section{Supplementary figure 12}
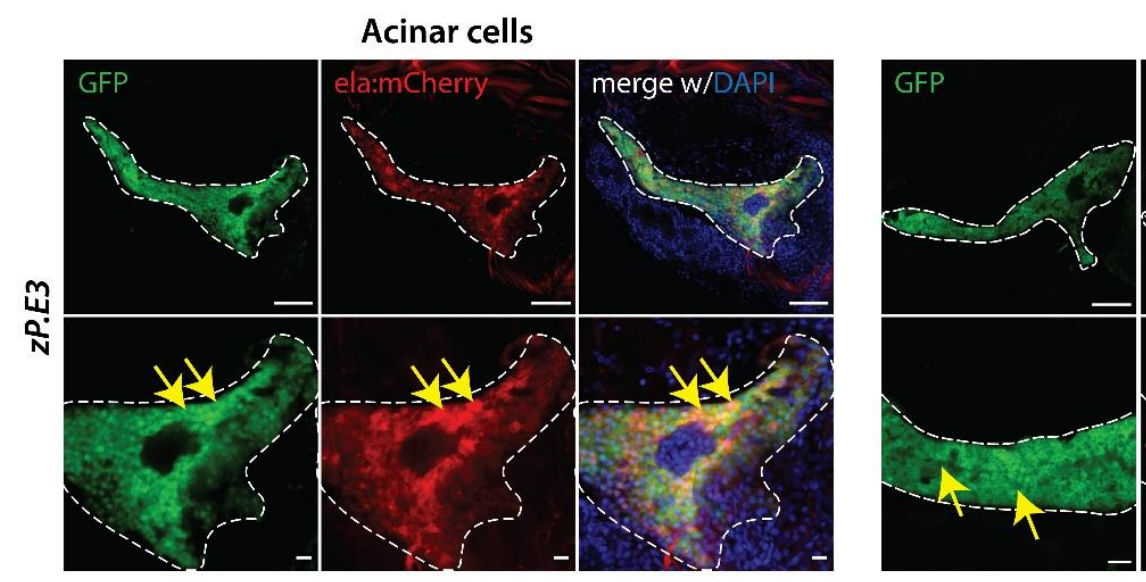

\section{Duct cells}
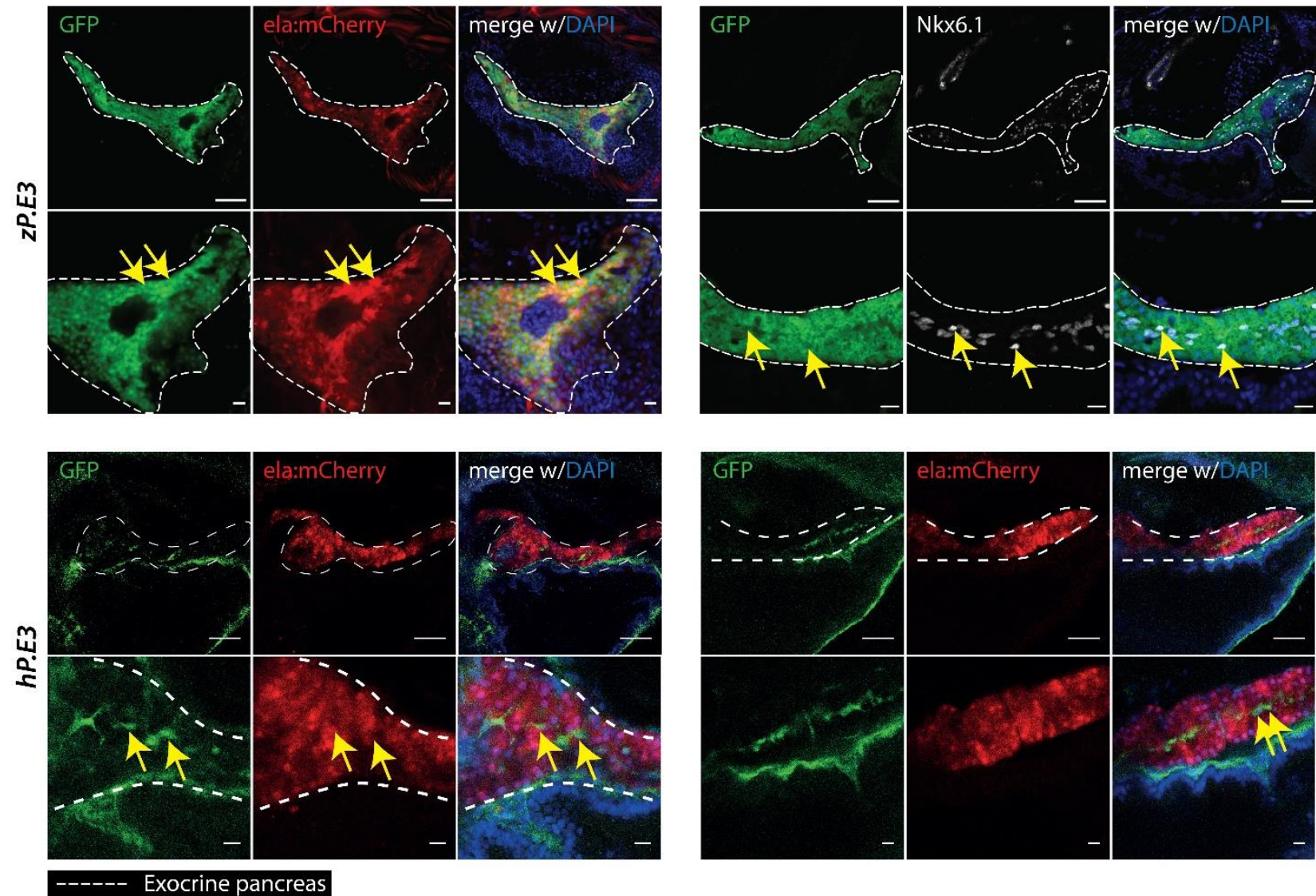

Supplementary Figure 12. Zebrafish and human distal ptfla/PTF 1a reporter transgenic lines, zP.E3 (zptf1aE3) and hP.E3 (hptf1aE3), respectively, drive GFP expression in pancreatic acinar cells and duct cells. Left: Representative confocal images of a double zebrafish reporter transgenic line (zE3:GFP and elastase:mCherry) and a human reporter transgenic line (hP.E3: GFP), showing zP.E3 and hP.E3 mediated GFP expression (green) co-localizing with the exocrine specific elastase mCherry mediated expression (red). Right: Representative confocal images of a double zebrafish reporter transgenic line (zE3:GFP and elastase:mCherry) co-localizing with Nkx6.1, a specific duct cell marker, and for the human reporter transgenic line (hP.E3: GFP), showing hP.E3 mediated GFP expression (green) not co-localizing with elastase:mCherry. 8dpf larvae were labeled with Nkx6.1 (white) and nuclei were labeled with DAPI (blue). Yellow arrows point to examples of GFP and pancreatic markers co-localization. Scale bar $60 \mu \mathrm{m}$. 


\section{Supplementary figure 13}

a

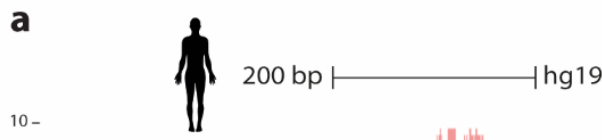

FOXA2

ChIP-seq
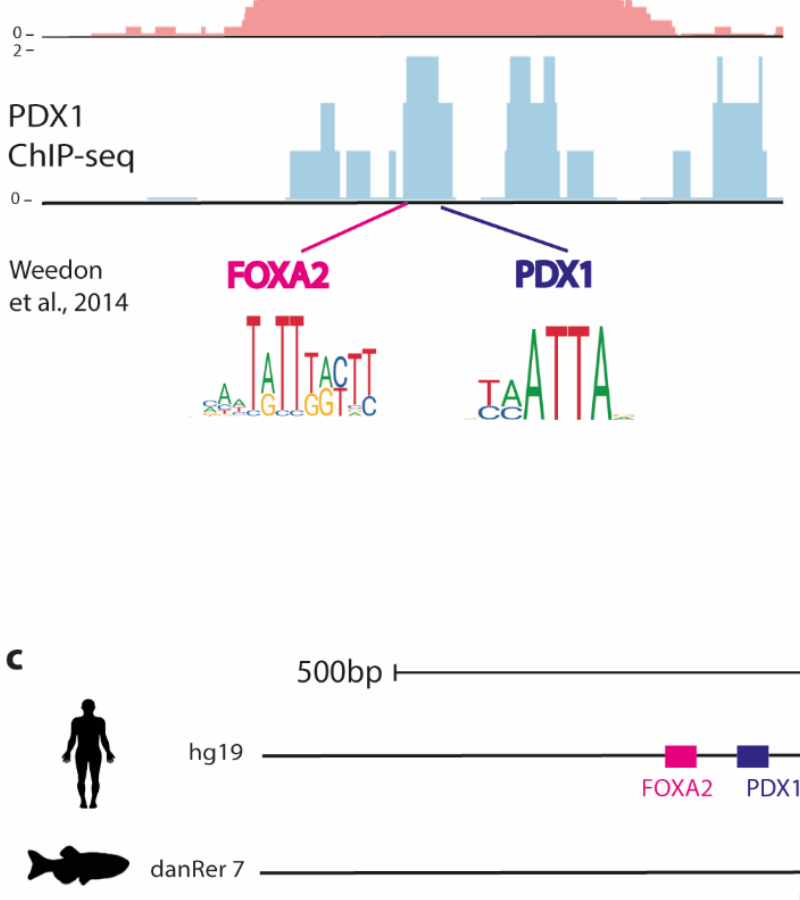

$500 \mathrm{bp}$ b

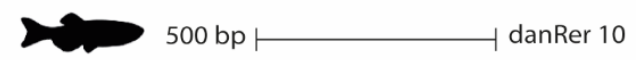

H3K27ac

ChiP-seq

57

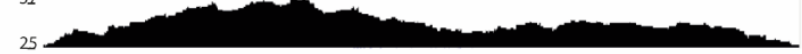

ATAC-seq

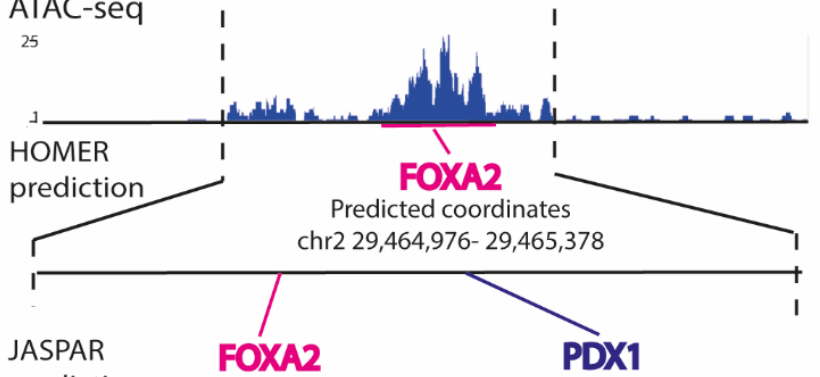

prediction

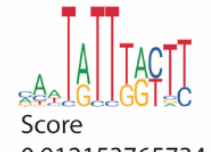

0.912153765734

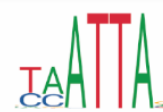

Score

0.965432863673

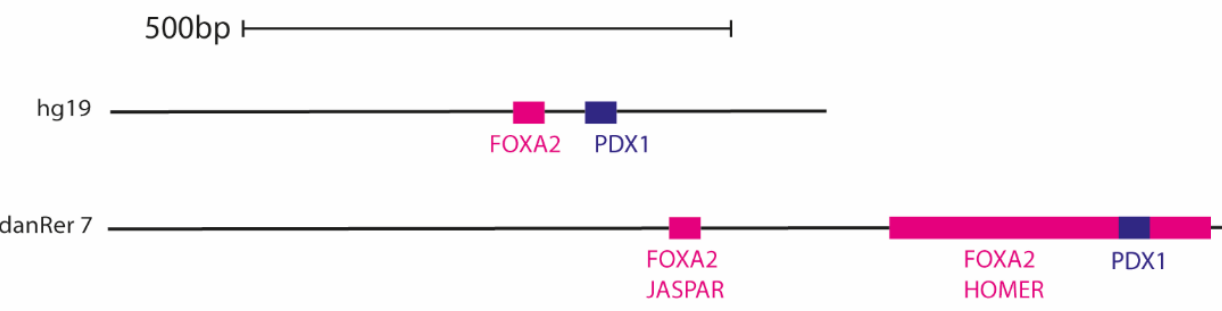

Supplementary figure 13. a) ChIP-seq density plots at the hP.E3 (hptf1aE3) locus showing FOXA2 (pink) and PDX1 (blue) ChIP-seq peaks generated from human endocrine islets ${ }^{4}$. The location of the respective predicted

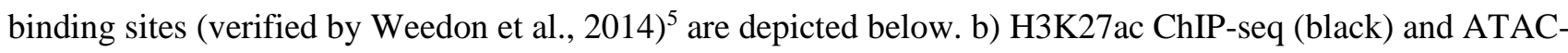
seq (blue) read density plots at the zP.E3 (zptf1aE3) locus, and putative FOXA2 and PDX1 transcription factor binding sites predicted by JASPAR ${ }^{6}$ with respective score. c) Schematic representation of the human and zebrafish ortholog enhancers; hP.E3 (above) and zP.E3 (below). 
bioRxiv preprint doi: https://doi.org/10.1101/2020.04.27.064220; this version posted April 28, 2020. The copyright holder for this preprint (which was not certified by peer review) is the author/funder. All rights reserved. No reuse allowed without permission.

\section{Supplementary figure 14}

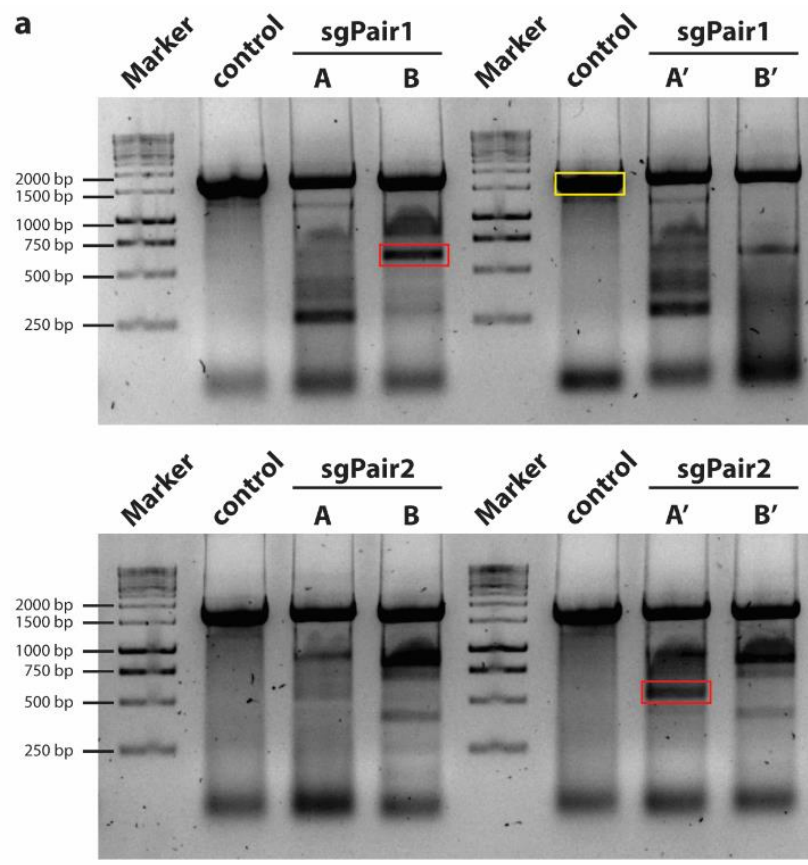

b

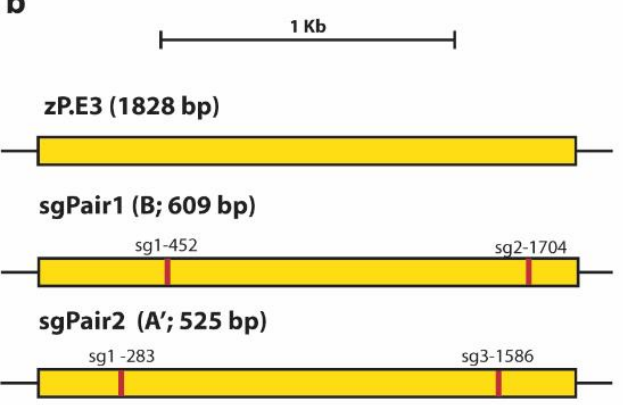

C

$+250$

ZP.E3

3'- CTCATGIGACCTGTCATCTGAAATAAATGAAAATTGCTATAAATAAAACAAAAACAATACATTAATTAGGATGCTTGGGCTITGTGTATAATACCTITGAAGTGCCTT

(1828 bp)

TGTCATCTGCCTCTAGTTGAGCTGTTCCAAATCCAGAGCTATGAACAAAAGAAAGAGAGAACATTGTITTGAAAAGATGAACCAGTGCAATATTIACTCCCCCAACT AATATTIAAAAAAGGCACAAAGACTGACATGGGAGCATAACGACAGGTCATCCAATTGTCTITGTTCATCTITGCAGCTTGGGTCAGTGGACTGAAGTCCAGTCCTGGC CATGACCTGCTCGGGCTCCAGGCCCTGACAAGCTTCTGACCTGGAGCACTGCTGTTGACACGTTCACTCACATGCACTGACCAAACACAAACCAATAAGTTCAAGGGCC TGTGITAATGCCCAAGTGCTITITITCCATAGAGACTTAAGAACTGTTCAGTCTTAIICAGTCACAGCCAATGCGAAAACGTITTAGCAGATGGTTCTTCGCATTCTGAAT AGATACATAAAAGGAGTTTATGTACCAATAAAATGCATTTCAAAAATAGATGTGTTCATGCATAATCACCAAAAACAGTGGCGTAAAATAGTCAGATGGTTACCAAAGT ATGCAATGAATGAACATCAAACACTATGAGAGATAAAGGCCTGACCCTTGAGAATACACCTGTCTTATGTTGCTICTCCAGECTCCCACAGAGTGTGAGATGATAGC ATGGTCTGACAAGTGTCCTCAAACATTGCGCTCCGAATGCGAACTACCTCACACACCAACACAGATAAGGGAATAAGTCTATATTICCAAAACAAGCATTCGTGACTAA GGTITGGTTCAAAACCCACTGGAAACATTCTAAAGGGGCCATGAACAGAGAAGGCAAAATTCCCTTGATCTITGACATGGGAGAGGTCACTCTATTATTAAAAACE TACTCAGAGCTTAAAACTAACTTGTAGTATAAAATCAGACTGCCAAAATGAGTGTGAAAAGTGTCACTTITTGCATTATAGTGTGGCTTGACCAATGACATTIITGT TCTGCATGACAAACACAAGAACTACTCAGAAACCAACTACAACAATATGTGTCCATGGTAAGTIITGCATTGTCATCTITCCTGATGCTAACATTAAGAAAGTGTGC ATITTGTTGCTGGAAAATGCTGGCCAACTATGCTGTGTCCGCTTGAGTAATATATTTATTATGTGGTCACTATTGCATTGTTGTTAGTACACCTAATTGAATATGTAT TATTTAAGCTIITTAACCTAAATCTCAGCAGCAACCTATCAAGAAAGTG TIIITITGCAGGTTAAAAGACCTACAAAAATTAAAAGAGTTACCCAATAATATCAGTGC TACTTCAATGTCTACATTTCACCATGCTACGTTGTACTGTGTGTTCAMGAAGATAAAACAGGACAGCTAACAGGCTAAAACA

sgPair1

CTCATGTGACCTGTCATCTGAAATAAATGAAAATGCTATAAATAAAACAAAAACAATACATTAATAGGATGCTTGGGCTTGTGTATAATACCTITGAAGTGCCT

(B; 609 bp) TGTCATCTGCCTCTAGTTTGAGCTGTTCCAAATCCAGAGCTATGAACAAAAGAAAGAGAGAACATTTGTITTGAAAGATGAAGCA

sgPair2

CTCATGTIGACCTGTCATCTGAAATAAATGAAA

(A'; 525 bp)

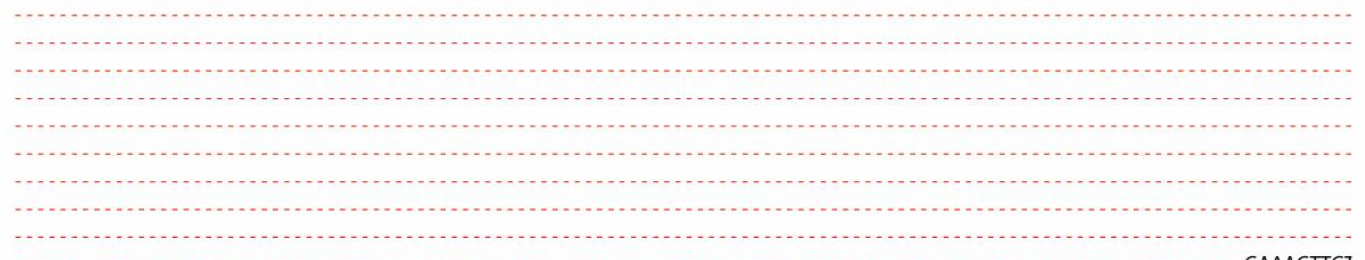

TIIIITGCAGGTIAAAAGACCTACAAAAATTAAAAGAGTTACCCAATAATATCAGTGGGCATTTACTTCAATGTCTACATTTCACCATGCTRGLACTGTGTGTTCTAATGA AGATAAAACAGGACAGCTAACAGGCTAAAACA

-3'

(1)

(1)

 2.  ATTGCATTGTाGTAGTACACCTAATGAATATGTATTCAATATTAAGCTIIITAACCTAAATCTCAGCAGCAACCTATCAAGAAAGTTGTIT GCAGGTTAAAAGACCTACAAAAATTAAAAGAGTTACCCAATAATATCAGTGGGCATTTACTTCAATGTCTACATTCACCATGCTACGTTGTACTGTGTGTTCTAATGAA AACAGGACAGCTAACAGGCTAAAACA 
Supplementary figure 14. CRISPR/Cas9-mediated deletion of the zP.E3 (zptf1aE3) enhancer in F0 zebrafish. a) PCR screening of zptf1aE3 deletion after co-injection of zebrafish embryos with Cas9 and different combinations of sgRNAs: successful deletions appear as truncated PCR products (red rectangle), in comparison with the wild-type sequence from non-injected embryos (control, yellow rectangle). b) Schematic representation of the deletions induced by CRISPR/Cas9. c) Sequence of the wild-type and representative sequences of enhancer deletion, corresponding to the annotated red rectangles in the gel (a). 
bioRxiv preprint doi: https://doi.org/10.1101/2020.04.27.064220; this version posted April 28, 2020. The copyright holder for this preprint (which was not certified by peer review) is the author/funder. All rights reserved. No reuse allowed without permission.

\section{Supplementary figure 15}
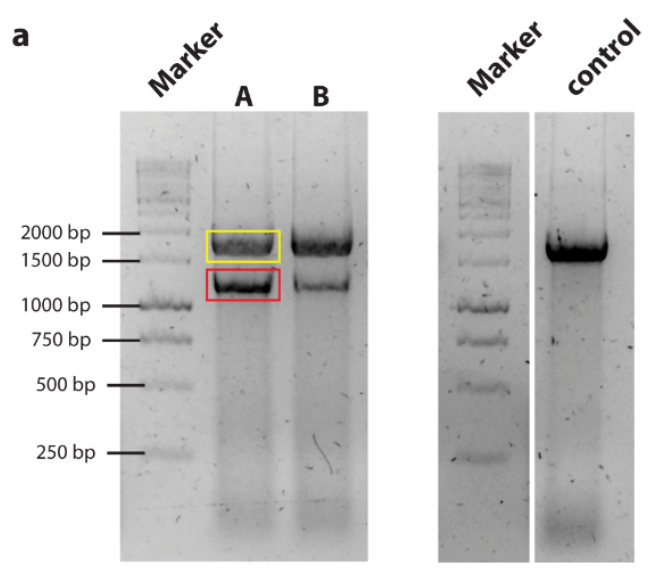

b

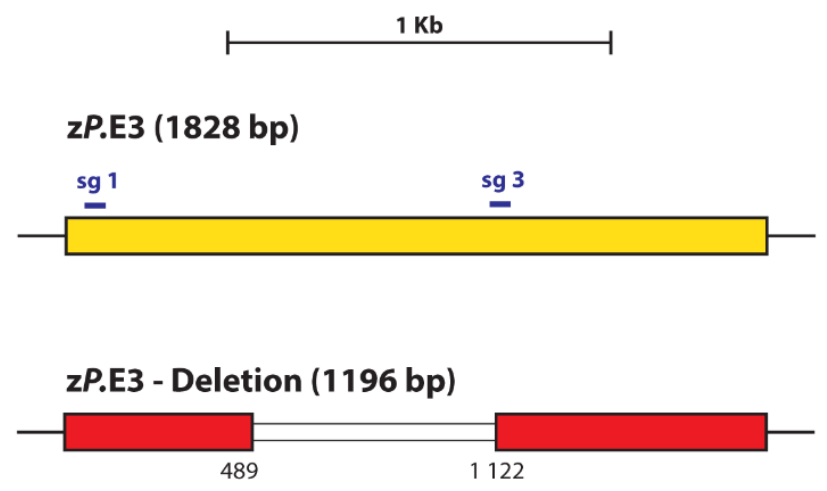

C

zP.E3 3'- CTCATGTGACCTGTCATCTGAAATAAATGAAAATTGCTATAAATAAAACAAAAACAATACATAAATAGGATGCTIGGGGCTITGTGTATAATACCTITGAAGTGCCT $(1828$ bp) TGTCATCTGCCTCTAGTTGAGCTGTTCCAAATCCAGAGCTATGAACAAAAGAAAGAGAGAACATTGITTGAAAAGATGAACCAGTGCAATATTACTCCCCCAACT AATATTAAAAAAGGCACAAAGACTGACARGGAGCATAACGACAGGTCATCCAATGTCTITGTCATCTITGCAGCTTGGGTCAGTGGACTGAAGTCCAGTCCTGGC CATGACCTGCTCGGGCTCCAGGCCCTGACAAGCTTCTGACCTGGAGCACTGCTGTTGACACGTTCACTCACATGCACTGACCAAACACAAACCAATAAGTTCAAGGGCC TGTGTTAATGCCCAAGTGCTITITITCCATAGAGACTTAAGAACTGITCAGTCTTAIICAGTCACAGCCAATGCGAAAACGTITIAGCAGATGGTTCTTGCATTCTGAAT AGATACATAAAAGGAGTTIATGTACCAATAAAATGCATTTCAAAAATAGATGTGTTCATGCATAATCACCAAAAACAGTGGCGTAAAATAGTCAGATGGTTACCAAAGT ATGCAATGAATGAACATCAAACACTATGAGAGATAAAGGCCTGACCCTTGAGAATACACCTGTCTTATGTTGCTITCTCCAGGCTCCCACAGAGTGTGAGATGATAGC ATGGTCTGACAAGTGTCCTCAAACATTGCGCTCCGAATGCGAACTACCTCACACACCAACACAGATAAGGGAATAAGTCTATATTTCCAAAACAAGCATTCGTGACTAA GGTाTGGTTCAAAACCCACTGGAAACATTCTAAAGGGGCCATGAACAGAGAAGGCAAAATTCCCTTGATCTITTGACATGGGAGAGGTCACTCTATTATTAAAAACG TACTCAGAGCTTAAAACTAACTTGTIAGTATAAAATCAGACTGCCAAAATGAGTGTGAAAAGTGTCACTTITIGGATTATAGTGTGGCTTGACCAATGACATIIITGT TCTGCATGACAAACACAAGAACTACTCAGAAACCAACTACAACAATATGTTGTCCATGGGTAAGTITTGCATTGTCATCTITCCTGATGCTAACATTAAGAAAGTGTGC ATTITGTTGCTGGAAAATGCTGGCCAACTATGCTGTGTCCGCTTGAGTAATATATTTATTATGTGGTCACTATTGCATTGTTGTTAGTACACCTAATTGAATATGTAT TATTIAAGCTITITAACCTAAATCTCAGCAGCAACCTATCAAGAAAGTG TITITITGCAGGTIAAAAGACCTACAAAAATTAAAAGAGTTACCCAATAATATCAGTGG TACTTCAATGTCTACATTICACCATGCTACGTGTACTGTGTGTTCAMGAAGATAAAACAGGACAGCTAACAGGCTAAAACA

zP.E3 -

3' - CTCATGTTGACCTGTCATCTGAAATAAATGAAAATGCTATAAATAAAACAAAAACAATACATTAATAGGATGCTTTGGGCTTTGTGTATAATACCTTIGAAGTGCCTT TGTCATCTGCCTIAGTITGAGCTGTTCCAAATCCAGAGCTATGAACAAAAGAAAGAGAGAACATTTGTTIGAAAAGATGAACCAGTGCAATATTIACTCCCCCAACTTC

Deletion AАТАТТТ-

(1196 bp)

(1)

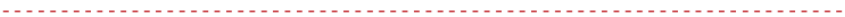
GGAATAAGTCTATATTICCAAAACAAGCATTCGTGACTAATGTIGGTITGGTTCAAAACCCACTGGAAAC TCTAAAGGGGCCATGAACAGAGAAGGCAAAATTCCCTTGATCTITTGACATGGGAGAGGTCACTCTATTATTAAEATCCAGTACTCAGAGCTTAAAACTAACTTGTTAGI TAAAATCAGACTGCCAAAATGAGTTGTGAAAAGTGTCACTTATTGCATTATAGTGTGGCTTGACCAATGACATIITITITAGTTCTGCATGACAAACACAAGAACTAC AAACCAACTACAACAATATGTTGTCCATGGTAAGTITTGCATTGTCATCTTCCTGATGCTAACATTAAGAAAGTGTGGATTAATITTGTTGCT BEA ECTGTGCCAAC TATGCTGTGTCCGCTTGAGTAATATATITATTATGTGGTCACTATTGCATGTTGTTAGTACACCTAATGAATATGTATCAATATTAAGCTIITIAACCTAAATCTC GCAACCTATCAAGAAAGTGTIIIITGCAGGTTAAAAGACCTACAAAAATTAAAAGAGTTACCCAATAATATCAGTGGGCATTACTTCAATGTCTACATTCACCATC GTTGTACTGTGTGITCTAATGAAGATAAAACAGGACAGCTAACAGGCTAAAACA

d

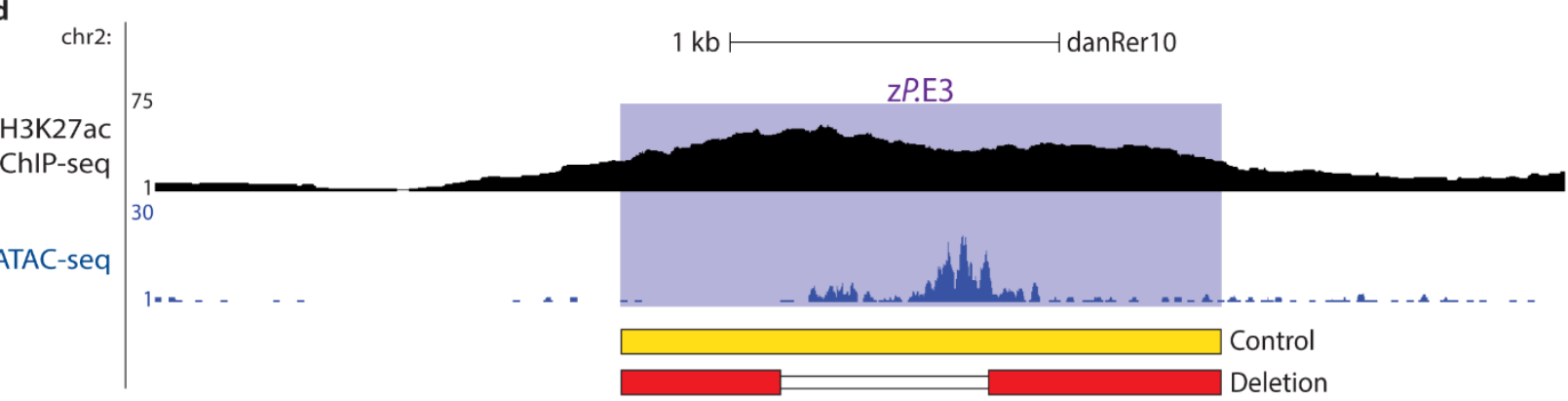


Supplementary figure 15. Mutant zebrafish with zP.E3 (zptf1aE3) enhancer deletion. a) PCR screening of genomic deletions for zP.E3 enhancer in zebrafish embryos, appearing as truncated PCR products (red rectangle), in comparison with the wild-type sequence from the same batch siblings (control; yellow rectangle). b) Schematic representation of the 632 bp deletion induced by CRISPR/Cas9. c) DNA sequencing results of the indicated genomic PCR products (yellow and red rectangles). d) Alignment of the wild-type and deleted alleles to the zebrafish, genome showing the ATAC-seq and ChIP-seq profiles at the zP.E3 locus. 


\section{Supplementary figure 16}

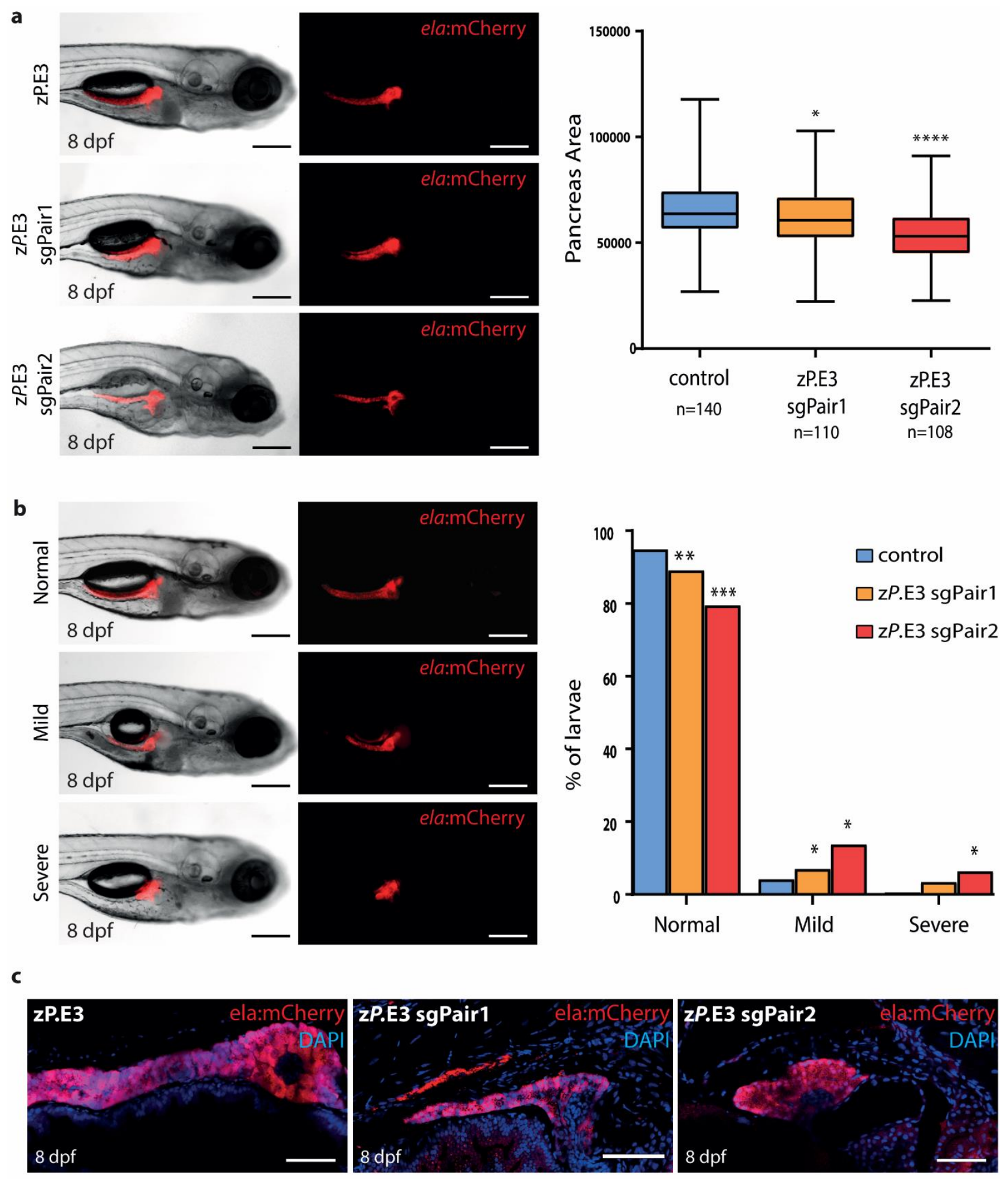


Supplementary figure16. CRISPR/Cas9-mediated deletion of zP.E3 (zptf1aE3) affects pancreas size in F0 zebrafish. a) $\operatorname{Tg}$ (elastase:mCherry) embryos were injected with Cas9 alone (control) or co-injected with Cas9 and a pair of sgRNAs (zP.E3 sgPair1 or sgPair2) and monitored at 8 dpf. Representative live images are shown in the left panels. Scale bar: $250 \mu \mathrm{m}$. Quantification of total pancreas area are represented in the right panels. Unpaired student's t-test (two-tailed), $\mathrm{p}$-values $<0.05$ were considered significant $(* * * * p<0.0001, * \mathrm{p}<0,05)$. b) Larvae where phenotyped as either normal or as one of the two following classes: mild pancreatic defect with significantly reduced pancreas size (mild) and severe pancreatic defects with reduced pancreas with abnormal structure (severe). Fisher's exact test, p-values $<0.05$ were considered significant $(* * * p<0.001, * * p<0.01$, $\left.{ }^{*} \mathrm{p}<0.05\right)$. c) Representative confocal fluorescent images of whole-mounted $8 \mathrm{dpf} \operatorname{Tg}($ elastase:mCherry) larvae showing impaired development of pancreas upon CRISPR/Cas9-mediated deletions of the zP.E3 enhancer (zP.E3 sgPair1 and zP.E3 sgPair2), in comparison to the control, injected with Cas9 alone (zP.E3). The elastaseproducing acinar cells of the exocrine tissue express mCherry (red) and nuclei are stained with DAPI (blue). Scale bar, $60 \mu \mathrm{m}$. 


\section{Supplementary figure 17}
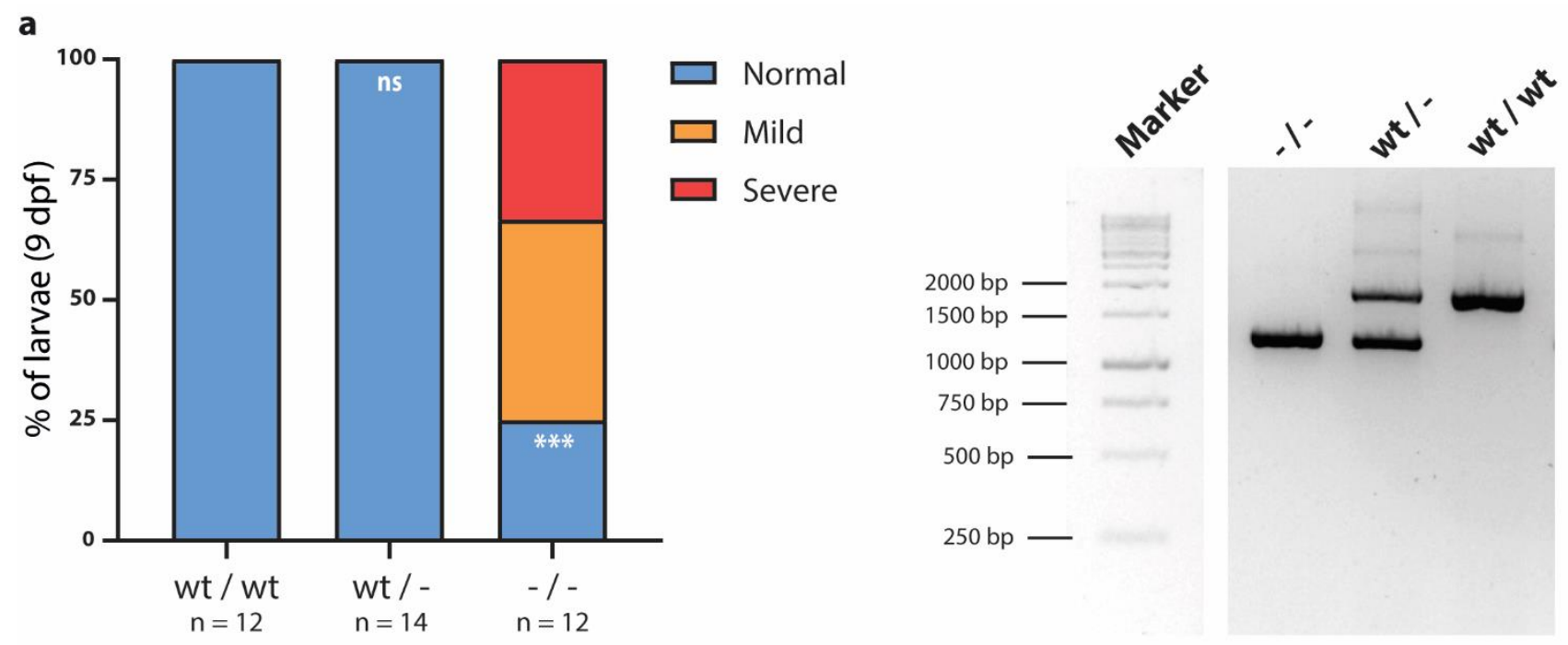

b
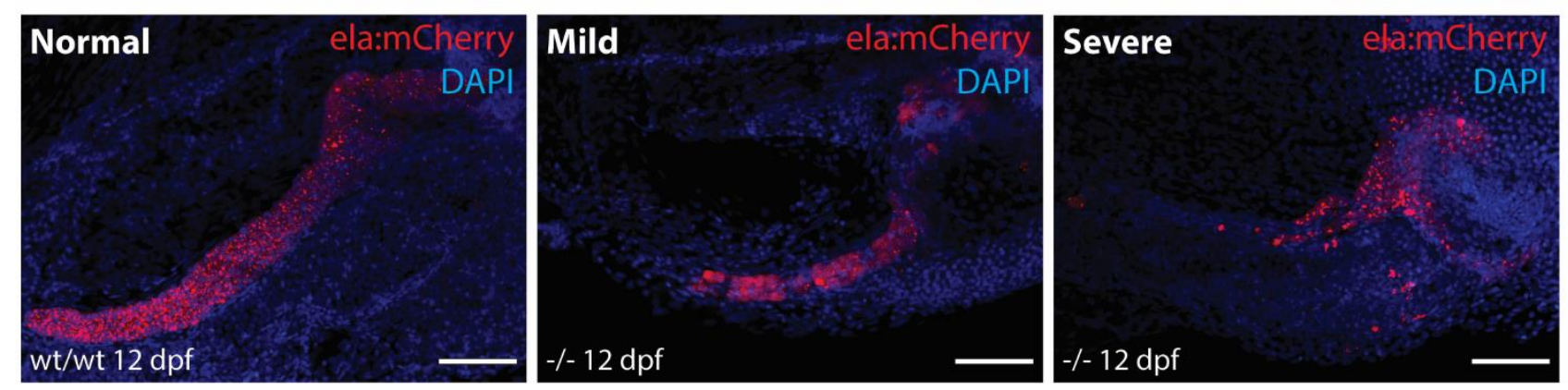

Supplementary Figure 17. Homozygous deletion mutation in zP.E3 (zptf1aE3) affects pancreas size in F2 larvae. a) Homozygous embryos for the zP.E3 deletion (-/-) show mild to severe pancreatic defects, unlike wild type (wt/wt) and heterozygous (wt/-) sibling embryos (left). PCR screening of zPtf1aE3 deletion in F1 zebrafish embryos: wt/wt, wt/- and -/-. b) Representative confocal images (maximum intensity projections) of $12 \mathrm{dpf}$ $\operatorname{Tg}$ (elastase:mCherry) larvae showing impaired development of pancreas upon CRISPR/Cas9-mediated deletions in $z P . E 3^{-/-}$larvae compared to the control (zP.E3 ${ }^{\mathrm{w} / \mathrm{wt}}$ ). The elastase-producing acinar cells of the exocrine tissue express mCherry (red) and nuclei are stained with DAPI (blue). Scale bar, $60 \mu \mathrm{m}$. 


\section{References}

1. Hiller, M. et al. Computational methods to detect conserved non-genic elements in phylogenetically isolated genomes: application to zebrafish. Nucleic Acids Res. 41, e151 (2013).

2. White, R. J. et al. A high-resolution mRNA expression time course of embryonic development in zebrafish. Elife 6, (2017).

3. Whyte, W. A. et al. Master transcription factors and mediator establish super-enhancers at key cell identity genes. Cell 153, 307-319 (2013).

4. Pasquali, L. et al. Pancreatic islet enhancer clusters enriched in type 2 diabetes risk-associated variants. Nat. Genet. 46, 136-143 (2014).

5. Weedon, M. N. et al. Recessive mutations in a distal PTF1A enhancer cause isolated pancreatic agenesis. Nat Genet 46, 61-64 (2014).

6. Khan, A. et al. JASPAR 2018: update of the open-access database of transcription factor binding profiles and its web framework. Nucleic Acids Res. 46, D1284 (2018). 